Graduate Institute of International and Development Studies International Economics Department Working Paper Series

\title{
Can Countries Rely on Foreign Saving for Investment and Economic Development?
}

\author{
Eduardo Cavallo \\ Inter-American Development Bank \\ Barry Eichengreen \\ University of California, Berkeley, NBER, and CEPR \\ Ugo Panizza \\ The Graduate Institute, Geneva and CEPR
}

Chemin Eugène-Rigot 2

P.O. Box 136

CH - 1211 Geneva 21

Switzerland

(C) The Authors. All rights reserved. No part of this paper may be reproduced without the permission of the authors. 


\title{
Can Countries Rely on Foreign Saving for Investment and Economic Development?
}

\author{
Eduardo Cavallo \\ Barry Eichengreen \\ Ugo Panizza*
}

\begin{abstract}
A surprisingly large number of countries have been able to finance a significant fraction of domestic investment using foreign finance for extended periods. While many of these episodes are in low-income countries where official finance is more important than private finance, we also identify a number of episodes where a substantial fraction of domestic investment was financed via private capital inflows. That said, we find that foreign savings are not a good substitute for domestic savings. More often than not, episodes of large and persistent current account deficits do not end happily. Rather, they end abruptly with compression of the current account, real exchange rate depreciation, and a sharp slowdown in investment. We conclude that financing growth and investment out of foreign savings, while not impossible, is risky.
\end{abstract}

JEL Codes: F32, O16

Keywords: Current account, Growth, Volatility, Savings

\footnotetext{
* Cavallo, Inter-American Development Bank (cavalloe@iadb.org), Eichengreen, University of California, Berkeley, NBER, and CEPR (eichengr@berkeley.edu), Panizza, The Graduate Institute, Geneva and CEPR (ugo.panizza@graduateinstitute.ch). We would like to thank Kailin Chen and Matías Marzani for research assistance.
} 
Where in a closed economy investment is limited by saving, open economies can invest more than they save by tapping foreign finance. In theory, a poor country with a low saving rate but good growth prospects can build up its capital stock by running a large and sustained current account deficit. The question is whether this is feasible and productive in practice.

According to urban legend, the IMF has long regarded current account deficits greater than 4 per cent of GDP as a danger sign: deficits above this threshold indicate that a country is exposing itself to the risk of a current account reversal and growth collapse when the capital inflow that previously financed the deficit comes to a sudden stop. Investors conscious of the risks will hence be reluctant to finance current account deficits persistently exceeding this threshold. Countries seeking to build up their capital stocks thus must rely on domestic savings in order to do so. This was the famous finding of Feldstein and Horioka (1980). It is said to be a lesson of China, which has more than fully financed exceptionally high investment rates out of domestic savings and thereby sustained near-double-digit growth for three decades.

Our analysis supports one part of this conventional wisdom but not the other. Contrary to the received wisdom, we identify a substantial number of countries since 1970 that have been able to run current account deficits in excess of 4 , or even 6 or 8 per cent of GDP for as long as 10 years. It has been possible historically, in other words, for countries to finance a significant portion of domestic investment out of foreign saving, in contrast to popular presumption.

To be sure, a substantial number of these episodes are in Sub-Saharan Africa, where official finance has been more important than private finance. Official finance tends to be larger, both relative to private capital flows and the size of the recipient country, and more stable, given its source and motivation. However, in a significant number of other episodes, including a number in Latin America and the Caribbean (LAC), large and persistent current account deficits have been financed with private capital inflows, especially foreign direct investment.

But how do large and persistent current account deficit episodes end? Here our results are more consistent with the conventional wisdom: typically, they do not end happily. Instead they end abruptly, with compression of the current account, real exchange rate depreciation, and a sharp slowdown in investment. 
And what of the growth and volatility effects of large and persistent current account deficits? Large and persistent deficit episodes are characterized by above-average growth in the first 4 to 5 years of the episode. However, this growth advantage is completely lost after 5 years. Over a twenty year period the total growth effect of large and persistent current account deficit turns negative. ${ }^{1}$ In addition, periods that follow episodes of large and persistent current account deficits are characterized by higher output volatility than in control-group cases. We conclude that while financing growth and investment out of foreign savings is not impossible, it is risky and does not yield a clear growth dividend. ${ }^{2}$

Our finding that reliance on foreign savings for domestic investment is more feasible than suggested by Feldstein and Horioka's original analysis, although it also comes packaged with very considerable risks, is consistent with three subsequent literatures. First, there is the literature adopting Feldstein and Horioka's original approach but analyzing data for subsequent decades. These studies tend to show that the tight correlation between domestic saving and investment found in the original Feldstein-Horioka study loosened after the authors wrote. $^{3}$

Second, there is the literature on the years before 1913, when the savings-investment correlation was looser than in the third quarter of the $20^{\text {th }}$ century, and late developing countries like Canada, Australia, and Argentina relied substantially on foreign savings for domestic investment. Bayoumi (1990) and Taylor (1994) show that data like those used by Feldstein and Horioka look very different in this earlier period. As factors facilitating large, persistent and relatively stable flows of foreign finance, Fishlow (1985) and Eichengreen (1995) observe that much of the investment so financed was in infrastructure and tradablegoods capacity where it could be used to generate the exports needed to service the additional external debt, and that borrowing took place in a period of strong political links between the lenders (often European) and borrowers (often overseas regions of recent European settlement).

Third, there is the literature on sudden stops, which similarly highlights the risks of heavy reliance on private capital inflows (Calvo, Izquierdo and Mejia, 2004, Cavallo and Frankel, 2008). Sudden stops are when foreign investors dump domestic assets and/or local

\footnotetext{
${ }^{1}$ Although the difference in long-run growth between episodes and non-episodes is not always statistically significant.

${ }^{2}$ Reinhart and Trebesch's (2015) suggest that Greece's long history of debt crisis is a classic example of the pitfalls of relying on external financing.

${ }^{3}$ For a survey see Apergis and Tsoumas (2009).
} 
investors engage in capital flight. Sudden stops reduce or eliminate the external financing available to countries that invest more than what they save. As a result, the affected country has to abruptly close any outstanding current account deficit. This is usually done through a combination of real exchange rate depreciation and import contraction accompanied by recession. Real exchange rate depreciation can be particularly disruptive because it raises the cost of servicing foreign-currency-denominated debt, triggering bankruptcies and causing the country to incur large output costs. Sudden stops and the ensuing current account reversals are thus costly and require painful adjustment.

\section{$2 \quad$ Large and persistent current account deficits}

We analyze large and persistent current account deficit episodes in a panel of developing and advanced economies between 1970 and 2013. Our sample includes 24 advanced and 121 developing and emerging market countries. Of the developing countries, 35 are in SubSaharan African, 27 in Latin America and the Caribbean, 24 in Emerging Europe, 19 in Asia, and 16 in the Middle East and North Africa region (Table 1). The sample is unbalanced: in 1975 we have data for only 54 countries (19 advanced economies, 13 in Sub-Saharan Africa and 10 in Latin America and Caribbean). Only after 1978 are there more than 100 countries in the sample.

We define a current account deficit as persistent when it lasts for at least 10 years and as large when the average deficit is greater than 4, 6, 8 or $10 \%$ of GDP. To ensure that our sample does not include episodes with large current account swings, we only classify episodes as persistent when the average deficit is larger than the threshold listed above and in addition when there are no years with current account deficits smaller than $50 \%$ of that threshold. Because it is possible for overlapping periods to satisfy our definitions, and since the presence of overlapping episodes would complicate the statistical analysis, we build a dataset of non-overlapping episodes by selecting, among possible candidates, the episode with the largest average current account deficit.

Using this approach, we identify 90 4\% episodes, 56 6\% episodes, 39 8\% episodes, and 25 10\% episodes. ${ }^{4}$ The resulting list of episodes is in the appendix.

\footnotetext{
${ }^{4}$ Table A1 in the appendix provides the full list.
} 
Following Eichengreen and Panizza (2016), we build our control group using all nonoverlapping ten-year periods between 1970 and 2013 (1970-79; 1980-89; 1990-99; 2000-10) that: (i) do not overlap with one of the deficit episodes and (ii) do not overlap with any other period for which the ten-year average of the current account deficit exceeds the threshold. The sum of episodes and control-group cases gives the total number of usable observations for each selected threshold. When we use the $4 \%$ threshold, for example, we have 250 observations (90 episodes and 160 control-group periods), while when we use the $10 \%$ threshold we have 317 observations (25 episodes and 292 control-group periods).

We find that $4 \%$ episodes are not uncommon: $36 \%$ of our observations are classified as such episodes (Table 2). Sub-Saharan Africa and Emerging Europe have the greatest prevalence of such episodes, but we also find a large share of episodes in other developing regions and in advanced economies. By comparison, the share of $4 \%$ episodes is very high (73\%) in low income countries (most low income countries in the sample being in SubSaharan Africa).

Using the $6 \%$ threshold, $20 \%$ of observations qualify as episodes. We still find that a large number of episodes (more than a third) are in Sub-Saharan Africa and Emerging Europe. In other developing regions, in contrast, the share of episodes is often less than $15 \%$, and in the advanced economies it is just $10 \%$. In low income countries the share of $6 \%$ episodes is close to $50 \%$.

When we apply an $8 \%$ threshold, $13 \%$ of observations qualify as episodes, with a relatively large number of large and persistent deficit episodes in Sub-Saharan Africa, Emerging Europe, and low income countries (about 30\% of observations) and a moderate number of episodes in Latin America and the Caribbean (10\% of observations). There are very few episodes in Asia, Middle East and North Africa and the advanced economies (the share of episodes ranges between 2 and $6 \%$, and the number of episodes is never greater than $3)$.

Predictably, in the case of the $10 \%$ threshold, large and persistent current account deficits are relatively rare. There are only 25 such episodes (8\% of observations in our sample). These 10\% episodes are concentrated in Sub-Saharan Africa and Emerging Europe. There are no 10\% episodes in Asia and in the advanced economies, just 2 in the Middle East and North Africa, and just 4 in in Latin America and the Caribbean (one in Central America and three in the Caribbean). 
Are large and persistent current account surpluses more likely in countries that receive large official flows, defined as situations in which more than $30 \%$ of the current account deficit is financed with official flows $?^{5} 56 \%$ of periods characterized by large official flows overlap with 4 per cent episodes. Focusing on 6\% episodes, we find a $28 \%$ overlap with periods characterized by large official flows, and for 8 and 10\% episodes the overlaps with large official flows periods are 16 and $11 \%$, respectively.

A problem with scaling official flows by the current account is that we may have large ratios not because official inflows are large but because the deficit (the denominator) is small. We therefore also look at cases where net official inflows are greater than $30 \%$ of the thresholds used to build the episodes. ${ }^{6}$ When scaling official flows by GDP, we find a $71 \%$ overlap between of large official flows periods and 4\% episodes, the overlaps are $52 \%$ for $6 \%$ episodes and 31 and 24\% for 8 and 10\% episodes, respectively.

\section{The correlates of large and persistent current account deficits}

As a first step in examining the correlates of large and persistent current account deficits, we look at the separate effects of exports and imports, which typically dominate the current account. We do not find a statistically significant difference in export-to-GDP ratios between our episodes and control group cases (Table 3). In contrast, episodes have import-to-GDP ratios 12 to 21 percentage points higher than the control group. This is unsurprising: finance for the current account deficit allows countries to import more. Still, that the difference in imports relative to GDP is very large is striking and noteworthy.

DeLong and Summers (1991) found that investment in equipment is a key driver of economic growth. We therefore check whether countries with large and persistent deficits use foreign savings to finance the imports of machinery and equipment. This is not the case as it happens. Large and persistent current account deficits are actually associated with lower imports of machinery relative to GDP. The difference between episodes and control group cases ranges between 4 and 7 percentage points and is statistically significant at the 1 per cent confidence level. The higher import ratio of countries that rely on foreign savings is fully

\footnotetext{
${ }^{5}$ We use total net official flows and divide them by the current account balance. We set this variable equal to zero for country-years with negative official flows or a current account surplus.

${ }^{6}$ These values are $1.2 \%$ of GDP when we look at $4 \%$ current account deficits, $1.6 \%$ of GDP for $6 \%$ current account deficits, 2.4\% of GDP for $8 \%$ current account deficits, and 3\% of GDP for $10 \%$ current account deficits.
} 
offset by the lower share of imports of machinery. It follows that imports of machinery (as a share of GDP) are essentially identical in treatment and control group cases. Further results below cast doubt on whether foreign finance has sustained faster rates of economic growth. The failure of countries to use that foreign finance to boost imports of equipment and machinery may be part of the explanations.

In contrast, large and persistent current account deficits are associated with aboveaverage FDI inflows. While portfolio inflows are also larger than control group cases, the difference is not large, and it is never statistically significant for developing countries. ${ }^{7}$

Countries running current account deficits should be accumulating foreign liabilities, and in fact we find that deficit episodes are associated with lower net foreign assets. These net foreign assets can take a variety of forms, however, some of which do not vary between the treatment and control groups. For example, there is no difference in international reserves, on average, between treatment and control-group cases.

There is also no statistically significant correlation between the likelihood of observing an episode on the one hand and the level of the real exchange rate, capital account openness, the terms of trade, and GDP growth on the other. However, the averages in Table 3 mask more complicated dynamic behavior of these variables, as we show below. In fact, the first years of deficit episodes are typically characterized by relatively high growth and real appreciation, while the final years are characterized by relatively low growth and real depreciation. Similarly, reserves are accumulated in the early years of deficit episodes but drawn down later.

Deficit episodes are most prevalent in poor countries. GDP per capita is $\$ 7,000$ $\$ 9,000$ lower in the treatment group than the control group. In the subsample of developing countries the difference is, perhaps predictably, smaller (\$500 to $\$ 2,000)$ and not statistically significant. $^{8}$

Countries experiencing deficit episodes have investment rates roughly 3 percentage points higher than control group cases. ${ }^{9}$ At the same time, they feature saving rates 8 to 10 percentage points lower than control cases. This suggests that, on average, countries running

\footnotetext{
${ }^{7}$ Whether large current account deficits financed mainly by FDI "turn out better" - whether are not followed by equally sharp changes in GDP growth-is a separate question, to which we turn in Section 4 below.

${ }^{8}$ This is in line with the results of Table 2 showing that episodes are more frequent in developing countries.

${ }^{9}$ Though the difference is not always statistically significant.
} 
large and persistent current account deficits have investment rates somewhat higher than the control group, but that their investment rate would have been much lower had they had not been able to tap foreign savings. Put another way, our large-and-persistent-deficit episodes reflect low savings rates more than high investment rates, compared to control-group cases

We also compute the difference between net investment income recorded in the balance of payments and the notional income that a country would obtain if it received (or paid) $5 \%$ interest on its net foreign assets (the return assumed by Hausmann and Sturzenegger, 2007). ${ }^{10}$ The likelihood of observing an episode is in fact positively correlated with this return differential.

Of particular interest is the correlation between episodes and two indicators of the importance of official financial flows. As a first indicator we use the share of the current account balance financed by net official inflows. We divide the net official inflows by the current account deficit and set this indicator to zero for countries with a current account surplus and countries with net official outflows. In most cases episodes are characterized by a larger share of official finance, but the difference is statistically significant at the 5 percent level only for $4 \%$ episodes. As mentioned above, this finding may reflect fact that small current account deficits lead to high ratios even in the presence of limited official flows (because the current account deficit is the denominator). When we instead scale total net official flows (either positive or negative) by GDP, official flows are always significantly larger during episodes, confirming the importance of official finance.

For developing countries, we also consider the level and composition of external debt. Unsurprisingly, large and persistent current accounts deficit are associated with large debt stocks. The difference ranges from 30 to $47 \%$ of GDP and is statistically significant at conventional confidence levels. However, there are no differences in the shares of short- and long-term debt between treatment and control-group cases, although deficit episodes are characterized by larger shares of public and publicly-guaranteed external debt. This is another nail in the coffin of the Lawson-Robichek doctrine that countries can sustain large current account deficits so long as they are associated with private sector borrowing. Episodes are

\footnotetext{
${ }^{10}$ While there is much analysis of why some countries earn excess returns on their net foreign assets and whether these returns are sustainable (see inter alia Gourinchas and Rey 2007, Curcuru et al. 2007, Hausmann and Sturzengger 2007 and Eichengreen, 2004), we simply note that countries may be able to run larger current account deficits when the return on their gross foreign assets is higher than that on their gross foreign liabilities.
} 
also associated with a larger share of concessional debt, consistent with the high number of episodes in low-income countries.

\subsection{Soft landing or sudden stop?}

We now examine the behavior of a set of macroeconomic variables over the course of the deficit episode itself.

In Figures 1-4 the episode is the shaded area; we also report data one year before the beginning of the episode and one year following it. The solid line is the median during the episode, the dashed line is the overall median for episodes and tranquil periods alike.

In the presence of well-functioning capital markets, one expects that when countries use foreign savings to build up their capital stocks and then start using their newly accumulated capital and become richer, the current account deficit will start narrowing gradually. In practice, this does not seem to be the case. Evidently, episodes do not end because countries are growing up and out of their deficits, but they end because countries abruptly lose access to credit. A partial exception is episodes with very large (in excess of 10 per cent of GDP) current account deficits (Figure 4), where adjustment, while still steep, is more gradual. This presumably is due to the fact that 15 of 25 very large deficit episodes are in poor (Sub-Saharan African) countries. These countries often lack capital market access and finance their deficits with more stable official flows (see above), making for more gradually adjustments.

The conclusion that deficit episodes end when countries lose access to market-based finance is also supported by the fact that investment, GDP growth, and imports all collapse toward the end of the episode. These are classic signs of a sudden stop in capital flows.

To learn more about the financing mechanism, we use balance of payment accounts to decompose the net capital inflows associated with large and persistent deficit episodes. Specifically, we divide net inflows into capital account balance; net errors and omissions and the four main categories of the financial account balance. ${ }^{11}$ We describe the composition of

\footnotetext{
${ }^{11}$ Balance of payments accounting distinguishes three main sources of external financing: (i) Capital transfers (for example grants and debt forgiveness by creditors) which are recorded in the capital account of the balance of payment; (ii) liabilities creating capital inflows (direct investment, portfolio investment, other investment, and changes in reserve assets) which are recorded in the financial account of the balance of payments; and (iii) net errors and omissions which is a residual category to insure that the balance of payments sums to zero.
} 
net inflows starting 5 years before the inset of the episode and going all the way to 15 years after the inset of the episode.

By comparing the overall current account deficit (the solid line in Figure 5) and the various financing components of the deficit (the colored bars in Figure 5), we find that the two main sources of financing are "net direct investment" and "net other investment". Of the two main sources of financing, net direct investment is more stable and remains positive after the episode ends while "net other investment" dries-up (and turns into a net outflow) at the end of the episode.

The figure also shows that international reserves increase during the first 7 years of episodes (i.e., there are negative changes in reserves-to-GDP ratio). This is an indication that during the boom years capital inflows are larger than the current account deficit and that the authorities are doing at least something to limit the tendency for the economy to overheat. However, reserves starts falling towards the end of the episode. This finding is consistent with the idea that the central bank is trying to offset the impact of the initial decline in private capital inflows.

\section{2 Regression analysis}

We now estimate probit models where the units of observation are the ten-year periods described above and the dependent variable takes a value of one during episodes and zero otherwise. Without an instrumental variables strategy we cannot make strong claims of causality. The statistical analysis of this section just allows us to describe the conditional correlation between current account episodes and a large set of variables (in an effort to do more we use identification by heteroscedasticity below).

We start by regressing the dependent variable on a set of regional dummies. Consistent with Table 2, we find that $4 \%, 6 \%$ and $8 \%$ episodes are more likely in SubSaharan Africa and Emerging Europe, while there is no statistically significant difference for advanced economies (the excluded category), Asia, Middle East and North Africa, and Latin America and Caribbean (columns 1, 3, and 5 of Table 4). ${ }^{12}$

Table 3 showed that deficit episodes are more likely in poorer economies, and the list of episodes in Table A1 suggests that episodes are more likely in small economies. In

\footnotetext{
${ }^{12}$ The results of columns 7 and 8 and difficult to interpret because, when we consider $10 \%$ episodes, the dependent variable becomes collinear with the advanced economies and Asian dummies.
} 
columns 2, 4, 6, and 8 of Table 4, we augment the model with country size (measured by the $\log$ of total GDP) and the level of economic development (measured by the log of GDP per capita). Country size is always negatively and significantly correlated with the likelihood of an episode (evidently, large countries find it more difficult to finance large, persistent deficits). The coefficient on income per capita is sometimes negative (4 and 6\% episodes), sometimes positive (8 and 10\% episodes) and rarely significant at conventional confidence levels. Interestingly, controlling for GDP and GDP per capita reverses the coefficients of most of the regional dummies; the exception is Emerging Europe, which remains positive but is no longer statistically significant. Conditional on country size and income per capita, we are now less likely to observe 4-8\% episodes in developing regions.

Next we drop the regional dummies and augment the model with measures of capital account openness, the savings rate, and terms of trade. We run regressions for the full sample (Table 5, columns 1, 3, 5, and 7) and the subsample of developing countries (columns 2, 4, 6, and 8). Our results for total GDP and GDP per capita are robust to controlling for these variables. Capital account openness is positively correlated with the likelihood of observing an episode, but its coefficient is only statistically significant for 4, 6, and 8\% (and not $10 \%$ ) episodes. ${ }^{13}$ Notice also that this measure is only significant when we include all countries.

Persistent current account deficits are negatively correlated with terms of trade shocks (although this coefficient is not statistically significant for $10 \%$ episodes). Recall again that the terms of trade were never significant in the univariate correlations of Table 3. The multivariate results are more intuitive insofar as theory and logic suggest that countries wish to borrow abroad in bad times. What is surprising here is that in at least some cases they can borrow abroad in face of adverse shocks for periods as long as ten years. Finally, our previous result that the likelihood of observing an episode is negatively correlated with domestic savings is robust to controlling for the variables in Table 5.

We also augment the model with FDI, portfolio inflows, and official inflows (all in net terms and scaled by GDP) and corroborate the finding of the univariate analysis that FDI and official inflows are positively associated with large and persistent current account deficits (Table A2 in the Appendix). The coefficients on these variables are not always statistically significant, however. They are essentially zero for $10 \%$ episodes. ${ }^{14}$ Consistent with the

\footnotetext{
${ }^{13}$ This is in line with Reinhardt et al.'s (2013) finding that capital account openness can partly explain the Lucas paradox.

${ }^{14}$ These results are not reported in the table but are available from the authors on request.
} 
textbook distinction between portfolio flows on the one hand and FDI and official flows on the other (Frankel and Rose 1996, Carlson and Hernandez 2002), countries that rely on portfolio inflows are less likely to experience a large and persistent current account deficit, presumably because portfolio flows are more prone to interruption. When we control for FDI, official inflows, and portfolio inflows, none of our other variables (including country size and saving rate) is significantly correlated with the likelihood of observing a $10 \%$ episode. $^{15}$ When we experiment with the return differential, which is sometimes seen as a proxy for the carry trade, we find that its coefficient is positive but only sporadically significant. ${ }^{16}$

We also find that our baseline results are also robust to controlling for regional dummies, developing country dummy and LIC dummy (Tables A5-A7 in the Appendix).

\section{$4 \quad$ Growth and volatility}

Figures 1-4 showed that large, sustained current account deficits give rise first to a burst of growth and then to a slump. In this section we analyze these dynamics in more detail.

\subsection{Growth during and after episodes}

To explore what happens to growth during and after large deficit episodes, we build impulse response functions using a methodology similar to Jordà's (2005) local projections method. Specifically, we estimate:

$$
G_{i, t, t+h}=\alpha+\beta^{h} E P I_{i, t}+X_{i, t} \Gamma^{h}+\varepsilon_{i, t+h}
$$

Here $t$ is the first year of either an episode or a control period, $G_{i, t, t+h}$ is average growth of country $i$ between period $t$ and period $t+h$ (with $h=\{1,2, \ldots, 20\}$ ), $E P I_{i, t}$ is a dummy

\footnotetext{
${ }^{15}$ Results not reported in the table.

${ }^{16}$ If we run separate regression for imports and exports (the main component of the current account, we find that that imports remain positively correlated with large and persistent current account deficits while exports are rarely significantly correlated with current account deficit, consistent with earlier findings (Tables A3-A4 in the Appendix). If we jointly include both variables we find that imports are always negative and statistically significant and exports always positive and statistically significant. This is not surprising because imports and exports are the most important components of the current account.
} 
variable that takes a value of 1 if $t$ is the first year of an episode and zero if $t$ is the first year of a control period, and $X_{t}$ is a set of controls.

In Equation (1), $\beta^{h}$ is the impulse response $h$ periods after the start of an episode. Since $G_{i, t, t+h}$ is average growth between $t$ and $t+h, \beta^{h}$ should not be interpreted as the effect of the episode in year $t+h$ (that interpretation would be valid had the dependent variable instead been: $\left.G_{i, t+h-1, t+h}\right)$. Instead, $\beta^{h}$ summarizes the average effect of the episode over the period starting at $t$ and ending at $t+h$. This is the equivalent of an accumulated impulse response function with the traditional VAR methodology.

We estimate four models. The first model does not include controls (we set $X_{i, t}=0$ ). In the second model we include the log of initial GDP per capita (to control for convergence), average years of education of the adult population (to control for human capital), and the saving rate (since we found that large and persistent episodes are more likely in countries with low saving rates). ${ }^{17}$ In the third model we add a dummy variable (OFF) that takes a value of one for periods characterized by high levels of official financial flows (where net official financial flows scaled by GDP are above the sample median), and the interaction between this dummy and the episode dummy. Thus we estimate:

$$
G_{i, t, t+h}=\alpha+\beta^{h}\left(E P I_{i, t} \times\left(1-O F F_{i, t}\right)\right)+O F F_{i, t}\left(\delta^{h} E P I_{i, t}+\theta^{h}\right)+X_{i, t} \Gamma^{h}+\varepsilon_{i, t+h}
$$

In this set-up, $\beta^{h}$ measures growth after episodes that took place in periods characterized by low official inflows, while $\delta^{h}$ measures growth after episodes that took place in periods characterized by high official inflows. We estimate equation (2) controlling for initial income and human capital.

In the fourth model we explore the role of FDI inflows and check whether episodes characterized by high FDI inflows are different from episodes characterized by low FDI inflows. In practice, we estimate equation (2) by replacing the OFF dummy with a dummy that takes a value of one in periods characterized by high FDI inflows. ${ }^{18}$

While the impulse responses obtained from Equations (1) and (2) cannot be interpreted as the causal effect of an episode on growth, they allow us to track what happens

\footnotetext{
${ }^{17}$ Results are essentially identical if we estimate this model without controlling for the saving rate.

${ }^{18}$ We define as high FDI inflows periods where FDI inflows relative to GDP are above the sample median.
} 
to growth over the course of an episode compared to tranquil periods. (We return to this issue of causality later in this section.)

Building these impulse-response functions requires estimating 20 regressions for each equation, since $h=20{ }^{19}$ In Figures $6-9$, the solid line is the point estimate while the dashed lines indicate $95 \%$ confidence intervals. ${ }^{20}$

In the model with no controls (Figure 6), 4 and 6\% episodes are associated with above-average growth in the first 3-4 years, but after the $5^{\text {th }}$ year there is no statistically significant difference between episodes and control periods. In both cases, the point estimates become negative (suggesting that episodes lead to lower growth in the long run), but the difference is never statistically significant. $8 \%$ episodes are similar, although the results are somewhat weaker (we find small positive and marginally significant growth in the first three years and then a declining and statistically insignificant effect with negative point estimates 10 years after the start of the episode). In the case of $10 \%$ episodes, growth during the episode itself is never significantly different from that in the control group, although we find that 20-year growth is negative and statistically significant at the 10 per cent confidence level (see Table A11).

In regressions controlling for convergence, human capital, and the saving rate (Figure 7) we again find that 10 per cent episodes are followed by lower growth at long horizons. The difference from the control group is again significant at the ten per cent confidence level for 20 year horizons (Table A15).

When we compare growth after episodes financed with official inflows (the grey lines in Figure 8) with growth after episodes financed by other means (the black lines in Figure 8), there is no difference for $4 \%$ episodes but we find slightly higher growth for 6 and $8 \%$ episodes not financed with official flows. For $10 \%$ episodes, we find higher long term growth for episodes financed with official flows, although the difference between the two

\footnotetext{
${ }^{19}$ Since we have four models and four thresholds, we estimate a total of 320 regressions. Full regression results are in Tables A8-A23 in the Appendix.

${ }^{20}$ For instance, the top left panel of Figure 6 plots the coefficients of the regressions reported in Table A8: when $h=1$, EPI has a positive and statistically significant coefficient (the point estimate is 0.0205 ), the coefficient remains positive and statistically significant until $h=4$, at $\mathrm{h}=5$ is positive but not significant, and at $h=6$ it becomes negative but still insignificant. For $h>6$, the coefficient remains negative but it is never statistically significant. The top-left panel of Figures 7, instead, plots the coefficient reported in Table A12. In this case, the coefficient is always positive but not statistically significant when $h>6$.
} 
types of episodes is never statistically significant. ${ }^{21}$ Thus, it is difficult to draw strong conclusions about differences in the growth effects of large deficits financed by official versus other types of flows.

Next, we consider FDI financing: the grey lines in Figure 9 plot post-episode growth in episodes where FDI inflows were above the sample median. For 4 and 6\% episodes, FDI flows seem to deliver higher post-episode growth. But as before the difference between the two paths is not statistically significant. For 8 and 10\% episodes there seem to be no difference between episodes with large and small FDI flows.

In Figure 10 we use the model that controls for convergence, human capital and the saving rate (Tables A12-A15) to compare the evolution of log GDP per capita in a controlgroup country (the solid line) with a treatment-group country with the same initial conditions. For 4, 6, and 8\% episodes there is basically no difference between episodes and controlgroup cases. For $10 \%$ episodes, we find that after 20 years the level of GDP is 17 per cent lower in deficit countries than in control-group cases.

Recall that 10 per cent episodes are concentrated in countries receiving official finance (mostly in Sub-Saharan Africa). Insofar as official finance is provided in response to country problems, lower growth at long horizons may reflect those problems (selectivity) and not the effects of extended periods of foreign finance per se. Given this, we attempt to identify how large and persistent current account deficits affect subsequent growth by using heteroskedasticity in the regression residuals to identify causal relationships, following Rigobon (2003) and Lewbel (2012).

Aassume that we are interested in estimating Equation (1), that the episode dummy is endogenous, and that $\boldsymbol{X}$ is a matrix of exogenous variables. If to the standard OLS assumptions, we add an heteroskedasticity assumption (i.e., we assume that $E\left(X u^{2}\right) \neq 0$, where $u$ is the error term in the equation in which the episode depends on growth), then we can use $X u^{2}$ as an instrument for $E P I$.

The resulting impulse responses functions, in Figure 11, again paint a largely negative picture of the growth effects of large and persistent current account deficits. We find no significant positive effect, not even in the short run, but large negative effects in the long run that are sometimes statistically significant at the 5\% confidence level (for 6 and 10 per

\footnotetext{
${ }^{21}$ In Figures 8 and 9 we only plot the first 15 period because our estimations become imprecise with volatile parameters for $\mathrm{h}>15$.
} 
cent episodes). The level of income is lower, not higher, after 20 years (compare the actual and counterfactual paths in Figure 10 above).

In sum, we do not find that large and persistent current account deficits are associated with higher long-term growth. If anything the opposite is true.

\section{2 Volatility}

So far we have shown that large and persistent current account deficits do not pay dividends in terms of long-run growth. We now check if there are costs in terms of output volatility by estimating the following GARCH model:

$$
\begin{aligned}
& G_{i, t+h}=\alpha+\delta R_{i}+\varepsilon_{i, t+h} \\
& \sigma_{i, t+h}=\phi+\psi E P I_{i, t}+\vartheta \sigma_{i, t+h-1}+\rho u_{i, t+h-1}^{2}+u_{i, t+h}
\end{aligned}
$$

In equation (3) we regress annual growth $h$ years after the episode on a set of regional dummies. In Equation (4) the variance of annual growth is a function of being within 20 years of the beginning of an episode (EPI), a GARCH (1) component and an ARCH (1) component. The GARCH and ARCH parameters $\vartheta$ and $\rho$ capture the persistence in output volatility, while $\psi$ captures differences in the annual volatility of GDP growth between years that follow episodes and control periods.

We estimate equations (3) and (4) by setting $h=\{1,2, \ldots, 20\}$ (this is average volatility during the full twenty years following the beginning of the episode or the beginning of the control period), $h=\{1,2, \ldots, 10\}$ (this is average volatility during the episode compared with average volatility in the first ten years of the control period), and $h=$ $\{11,12, \ldots, 20\}$ (this is average volatility in the ten years that follow the end of the episode compared with volatility in the 10 years that follow the end of the control period).

Examining 4\% episodes (Table 7, column 1) reveals no difference in output volatility over the full 20 year period (top panel of Table 7). However, the treatment group is less volatile during the episode itself (middle panel of Table 7) and more volatile afterwards (bottom panel of Table 7). Together with the results of the previous section, this suggests that relatively small (4\%) deficits deliver both higher growth and less volatility in the short run at the price of more volatility after the episode and no difference in long-run growth or the level 
of output. When we consider 6, 8 and 10\% episodes (Table 7 columns 2-4), current account deficits are associated with higher output volatility both during and after the episodes.

Overall, it would appear that large and persistent current account deficits deliver little if any gain in terms of growth and some pain in terms of additional volatility.

\section{Conclusion}

Large-scale foreign funding offers attractive opportunities for financing domestic investment but comes with considerable risks. To characterize the tradeoffs, we have analyzed episodes characterized by large and sustained current account deficits. There turn out to be a surprising number of such episodes. Since 1970 a substantial number of countries have been able to finance significant portions of domestic investment out of foreign saving. While a significant fraction of these episodes are in low-income countries where official finance is more important than private finance, we have also identified a number of episodes that have been financed with private capital inflows, contrary to received wisdom.

But our analysis also suggests that foreign funding is not a good substitute for domestic savings in the sense that large, persistent current-account-deficit episodes do not end happily. More often than not, they end with sharp compression of the current account, real exchange rate depreciation, and a slowdown in investment.

Moreover, whatever its short-term benefits, reliance on foreign savings delivers higher volatility together with sub-par long-run growth performance. We conclude that financing growth and investment out of foreign savings, while not impossible, is risky. 


\section{References}

Apergis, N. and C. Tsouimas (2009), "A Survey of the Feldstein-Horioka Puzzle: What Has Been Done and Where We Stand,” Research in Economics 63, pp. 64-76.

Bayoumi, Tamim (1990), "Savings-Investment Correlations: Immobile Capital, Government Policy or Endogenous Behavior?” IMF Staff Papers 37, pp. 360-387.

Calvo, Guillermo, Alejandro Izquierdo and Luis-Fernando Mejia (2004), “On the Empirics of Sudden Stops: The Relevance of Balance-Sheet Effects,” in Proceedings, San Francisco: Federal Reserve Bank of San Francisco (June).

Cavallo, Eduardo and Jeffrey Frankel (2006), "Does Openness to Trade Make Countries More Vulnerable to External Crises, or Less? Using Gravity to Establish Causality,” Journal of International Money and Finance 27, pp. 1430-1452.

Curcuru, Stephanie, Tomas Dvorak, and Francis Warnock (2007) “The Stability of Large External Imbalances: The Role of Returns Differentials,” NBER Working Paper No. 13074

Eichengreen, Barry (1985), “The Gold Standard Since Alec Ford,” in Barry Eichengreen and Marc Flandreau (eds), The Gold Standard in Theory and History, London: Routledge, pp. 187-206.

Eichengreen, Barry (2004) “Global Imbalances and the Lessons of Bretton Woods,” NBER Working Paper No. 10497.

Eichengreen, Barry and Ugo Panizza (2016), “A Surplus of Ambition: Can Europe Rely on Large Primary Surpluses to Solve its Debt Problem?” Economic Policy (forthcoming).

Fishlow, Albert (1985), "Lessons from the Past: Capital Markets during the $19^{\text {th }}$ Century and the Interwar Period,” International Organization 39, pp. 383-439.

Gourinchas, Pierre-Olivier and Hélène Rey (2007), "From World Banker to World Venture Capitalist: U.S. External Adjustment and the Exorbitant Privilege,” in Richard Clarida (editor), G7 Current Account Imbalances: Sustainability and Adjustment, University of Chicago Press, pp.11-66.

Hausmann, Ricardo and Federico Sturzenegger (2007). "The Missing Dark Matter in the Wealth of Nations and its Implications for Global Imbalances,” Economic Policy 22, pp.469518. 
IMF (2013). Sixth Edition of the IMF's Balance of Payments and International Investment Position Manual (BPM6), Washington, D.C.: IMF.

Jordà, Òscar (2005) "Estimation and Inference of Impulse Responses by Local Projections," American Economic Review 95, pp.161-182.

Reinhardt, Dennis, Luca Ricci, and Thierry Tressel (2013) "International capital flows and development: Financial openness matters," Journal of International Economics, 91(2), pp. 235-251.

Reinhart, Carmen and Christoph Trebesch (2015), "The Pitfalls of External Dependence: Greece, 1829-2015,” Brookings Papers of Economic Activity.

Taylor, Alan (1994), "Domestic Saving and International Capital Flows Reconsidered,” NBER Working Paper no. 4892. 
Table 1: Number of observations

\begin{tabular}{lccccc}
\hline & $\mathbf{1 9 7 0}$ & $\mathbf{1 9 8 0}$ & $\mathbf{1 9 9 0}$ & $\mathbf{2 0 0 0}$ & $\mathbf{2 0 1 0}$ \\
\hline SSA & 1 & 32 & 38 & 35 & 35 \\
Asia & 1 & 11 & 15 & 18 & 19 \\
MNA & 13 & 13 & 13 & 16 \\
LAC & 4 & 26 & 25 & 27 & 27 \\
EME & & 1 & 4 & 23 & 24 \\
AE & 4 & 24 & 23 & 24 & 24 \\
\hline Total & $\mathbf{1 4}$ & $\mathbf{1 0 7}$ & $\mathbf{1 1 8}$ & $\mathbf{1 4 0}$ & $\mathbf{1 4 5}$ \\
\hline
\end{tabular}

Table 2: Episodes and control periods

\begin{tabular}{l|cccc|cccc}
\hline & \multicolumn{4}{|c|}{$\begin{array}{c}\text { 4\% CA Deficit } \\
\text { Episodes }\end{array}$} & Control & \% Episodes & Total & \multicolumn{4}{c}{ 6p CA Deficit } \\
\hline ALL Countries & 90 & 160 & $36 \%$ & 250 & 56 & 222 & $20 \%$ & 278 \\
SSA & 31 & 22 & $58 \%$ & 53 & 23 & 37 & $38 \%$ & 60 \\
Asia & 10 & 23 & $30 \%$ & 33 & 4 & 30 & $12 \%$ & 34 \\
MNA & 6 & 16 & $27 \%$ & 22 & 4 & 27 & $13 \%$ & 31 \\
LAC & 17 & 38 & $31 \%$ & 55 & 9 & 53 & $15 \%$ & 62 \\
EME & 12 & 6 & $67 \%$ & 18 & 9 & 12 & $43 \%$ & 21 \\
AE & 14 & 55 & $20 \%$ & 69 & 7 & 63 & $10 \%$ & 70 \\
\hline LIC & 22 & 8 & $73 \%$ & 30 & 16 & 18 & $46 \%$ & 34 \\
Large Off. Flows to CA & 36 & 28 & $56 \%$ & 64 & 20 & 52 & $28 \%$ & 72 \\
Large Off. Flows to GDP & 49 & 20 & $71 \%$ & 69 & 30 & 28 & $52 \%$ & 58 \\
\hline & & $\mathbf{8 \%}$ CA Deficit & & & $\mathbf{1 0 \%}$ CA Deficit & \\
& Episodes & Control & \% Episodes & Total & Episodes & Control & \% Episodes & Total \\
\hline ALL Countries & 39 & 265 & $13 \%$ & 304 & 25 & 292 & $8 \%$ & 317 \\
SSA & 19 & 48 & $28 \%$ & 67 & 15 & 62 & $19 \%$ & 77 \\
Asia & 1 & 37 & $2 \%$ & 38 & 0 & 37 & $0 \%$ & 37 \\
MNA & 2 & 31 & $6 \%$ & 33 & 2 & 32 & $6 \%$ & 34 \\
LAC & 7 & 62 & $10 \%$ & 69 & 4 & 67 & $6 \%$ & 71 \\
EME & 7 & 16 & $30 \%$ & 23 & 4 & 19 & $17 \%$ & 23 \\
AE & 3 & 71 & $4 \%$ & 74 & 0 & 75 & $0 \%$ & 75 \\
\hline LIC & 11 & 27 & $29 \%$ & 38 & 8 & 36 & $19 \%$ & 44 \\
Large Off. Flows to CA & 14 & 72 & $16 \%$ & 86 & 10 & 84 & $11 \%$ & 94 \\
Large Off. Flows to GDP & 17 & 28 & $31 \%$ & 55 & 11 & 35 & $24 \%$ & 35 \\
\hline
\end{tabular}


Table 3: The correlates of large and persistent current account deficits

\begin{tabular}{|c|c|c|c|c|c|c|c|c|c|c|c|c|}
\hline & \multicolumn{3}{|c|}{ 4\% CA deficit } & \multicolumn{3}{|c|}{ 6\% CA deficit } & \multicolumn{3}{|c|}{ 8\% CA deficit } & \multicolumn{3}{|c|}{$10 \%$ CA deficit } \\
\hline & Episode & Control & Diff & Episode & Control & Diff All C & $\begin{array}{l}\text { Intries } \\
\text { Episode }\end{array}$ & Control & Diff & Episode & Control & Diff \\
\hline Current Account to GDP & -9.66 & 1.36 & $-11.01 * * *$ & -11.57 & -0.03 & $-11.54 * * *$ & -13.26 & -0.91 & $-12.35^{* * *}$ & -14.73 & -1.40 & $-13.33^{* * *}$ \\
\hline Exports to GDP & 34.93 & 36. 50 & -1.58 & 37.43 & 35. 42 & 2. 02 & 39. 30 & 35. 33 & 3.97 & 33. 97 & 35. 61 & -1.64 \\
\hline Imports to GDP & 47.39 & 35. 70 & 11. $69^{* * *}$ & 53. 03 & 36.40 & 16. $63^{* * *}$ & 58. 18 & 37.10 & 21. $09^{* * *}$ & 57.52 & 37. 98 & 19. $53^{* * *}$ \\
\hline Share of Machinery Imports & 22.9 & 27.1 & $-4.2 * * *$ & 21.8 & 26.3 & $-4.5^{* * *}$ & 19.9 & 26.0 & $-6.1^{* * *}$ & 18.5 & 25.8 & $-7.3^{* * *}$ \\
\hline Imports of Machinery to GDP & 10.4 & 9.9 & 0.5 & 11.1 & 9.6 & 0.5 & 11.4 & 9.6 & 0.8 & 9.9 & 9,8 & 0.1 \\
\hline Capital Account to GDP & 8.03 & -1.31 & 9. $34^{* * *}$ & 9.46 & -0.05 & 9. $51^{* * *}$ & 10.74 & 0.75 & 9. $99 * * *$ & 12. 05 & 1. 12 & $10.94 * * *$ \\
\hline Net FDI Inflows to GDP & 4. 25 & 2. 48 & $1.77^{* * *}$ & 4. 99 & 2. 41 & 2. $58^{* * *}$ & 5. 82 & 2. 49 & 3. $33^{* * *}$ & 6. 55 & 2. 51 & 4. $04^{* * *}$ \\
\hline Net Portfolio Investment to GDP & 0.40 & -0.42 & $0.81^{* * *}$ & 0.24 & -0.29 & $0.52 *$ & 0.01 & -0.18 & 0.18 & 0.02 & -0.16 & 0.18 \\
\hline Net Foreign Assets to GDP & -68.77 & -10.10 & $-58.67 * * *$ & -79.12 & -17.13 & $-61.99 * * *$ & -96.18 & -22.81 & $-73.36^{* * *}$ & -113.30 & -25.53 & $-87.77^{* * *}$ \\
\hline International Reserves to GDP & 13. 03 & 13. 24 & -0.21 & 14. 59 & 12. 70 & 1.89 & 15. 37 & 12. 53 & 2. 84 & 14. 31 & 12. 44 & 1. 87 \\
\hline Capital Account Openness & 0.47 & 0.55 & -0.07 & 0.48 & 0.52 & -0.04 & 0.49 & 0.50 & 0 & 0.44 & 0.50 & -0.06 \\
\hline Nominal XR & 1.17 & 1.13 & 0.04 & 1.24 & 1.13 & 0.11 & 1.23 & 1.11 & 0.12 & 1.25 & 1. 11 & 0.14 \\
\hline Real Effective XR & 0.99 & 1. 02 & -0.03 & 0.98 & 1. 01 & -0.03 & 0.98 & 1. 01 & -0.03 & 1. 01 & 1. 01 & 0 \\
\hline Terms of trade & -0.09 & -0.03 & -0.05 & -0.09 & -0.03 & -0.05 & -0.10 & -0.03 & -0.07 & -0.12 & -0.04 & -0.09 \\
\hline Real GDP Growth & 5. 44 & 4. 98 & 0.46 & 5. 45 & 4. 99 & 0.46 & 5. 53 & 4. 88 & 0.65 & 4. 86 & 4. 91 & -0.04 \\
\hline Saving Rate & 14. 38 & 23. 72 & $-9.33 * * *$ & 13. 87 & 21. 96 & $-8.09 * * *$ & 13. 21 & 21. 04 & $-7.83 * * *$ & 10.75 & 20. 39 & $-9.65^{* * *}$ \\
\hline Investment Rate & 23. 74 & 22. 36 & 1. $38^{*}$ & 24. 85 & 21. 93 & 2. $92 * * *$ & 25.13 & 22. 07 & 3. $06^{* * *}$ & 24.18 & 21.98 & 2. $2^{*}$ \\
\hline GDP per capita & 11619 & 19369 & $-7750 * * *$ & 9560 & 17704 & $-8144 * * *$ & 9396 & 16874 & $-7477^{* *}$ & 7057 & 16361 & $-9304 * *$ \\
\hline Return differential & 0.37 & -1.17 & 1. $54^{* * *}$ & 0.64 & -0.98 & 1. $61^{* * *}$ & 1.37 & -0.88 & 2. $24 * * *$ & 2. 40 & -0.80 & 3. $21^{* * *}$ \\
\hline Net official flows to CA & 28. 9 & 12.8 & 16. $1^{* * *}$ & 25.1 & 17.9 & 7. $2^{*}$ & 25.1 & 20.6 & & 25.1 & 22.0 & \\
\hline \multirow[t]{2}{*}{ Net official flows to GDP } & 2.3 & 0.4 & $1.9^{* * *}$ & 2.5 & 0.6 & $1.9^{* * *}$ & 2.8 & 0.8 & $2 * * *$ & 3.2 & 0.9 & 2. $3^{* * *}$ \\
\hline & \multicolumn{12}{|c|}{ Developing Countries } \\
\hline Current Account to GDP & -10.11 & 1.18 & $-11.29^{* * *}$ & -12.03 & -0.43 & $-11.61^{* * *}$ & -13.56 & -1.35 & $\frac{\text { Dint }}{-12.21 * * *}$ & $\begin{array}{l}\text { Eppisoue } \\
-14.73\end{array}$ & -1.88 & $\frac{\text { Dil1 }}{-12,85^{* * *}}$ \\
\hline $\begin{array}{l}\text { Exports to GDP } \\
\text { Ex }\end{array}$ & 34. 03 & 34.17 & -0.14 & 35.21 & 34.43 & 0.78 & 36. 52 & 34.29 & 2.23 & 33. 97 & 34.28 & -0.3 \\
\hline Imports to GDP & 47.93 & 34.46 & 13. $47^{* * *}$ & 52. 01 & 36.71 & 15. $3^{* * *}$ & 56. 31 & 37.35 & 18. $96^{* * *}$ & 57.52 & 37. 94 & 19. $58 * * *$ \\
\hline Share of Machinery Imports & 22.1 & 27.4 & $-5.3^{* * *}$ & 21.2 & 25.9 & $-4.7 * * *$ & 19.3 & 25.6 & $-6.4^{* * *}$ & 18.5 & 25.3 & $-6.8^{* * *}$ \\
\hline Imports of Machinery to GDP & 10.2 & 9.2 & 1.0 & 10.4 & 9.2 & 1.2 & 10.4 & 9.3 & 1.1 & 9.9 & 9.4 & 0.5 \\
\hline Capital Account to GDP & 8. 33 & -1.22 & 9. $55^{* * *}$ & 9.77 & 0.26 & $9.52^{* * *}$ & 11. 01 & 1. 09 & 9. $92 * * *$ & 12. 05 & 1. 47 & $10.58^{* * *}$ \\
\hline Net FDI Inflows to GDP & 4. 39 & 2. 25 & 2. $14^{* * *}$ & 5. 25 & 2. 27 & 2. $98^{* * *}$ & 5. 98 & 2. 36 & 3. $62 * * *$ & 6.55 & 2.38 & 4. $18^{* * *}$ \\
\hline Net Portfolio Investment to GDP & 0.20 & -0.13 & 0.32 & 0.09 & -0.13 & 0.23 & -0.08 & -0.08 & -0.01 & 0.02 & -0.07 & 0.09 \\
\hline Net Foreign Assets to GDP & -72.04 & -17.61 & $-54.43 * * *$ & -84.43 & -23.61 & $-60.82 * * *$ & -100.15 & -29.44 & $-70.71 * * *$ & -113.30 & -32.12 & $-81.18^{* * *}$ \\
\hline International Reserves to GDP & 13. 24 & 15. 44 & -2.2 & 14. 39 & 14. 43 & -0.04 & 14.94 & 13. 89 & 1.05 & 14. 31 & 13. 48 & 0.83 \\
\hline Capital Account Openness & 0.43 & 0.41 & 0.03 & 0.45 & 0.40 & 0.05 & 0.46 & 0.39 & 0.07 & 0.44 & 0.40 & 0.04 \\
\hline Nominal XR & 1. 18 & 1.22 & -0.04 & 1.24 & 1.19 & 0.05 & 1. 22 & 1. 16 & 0.06 & 1. 25 & 1. 15 & 0.1 \\
\hline Real Effective XR & 0.99 & 1. 02 & -0.04 & 0.98 & 1. 01 & -0.03 & 0.99 & 1. 02 & -0.02 & 1. 01 & 1. 02 & -0.01 \\
\hline Terms of trade & -0.10 & -0.04 & -0.05 & -0.10 & -0.04 & -0.06 & -0.11 & -0.04 & -0.07 & -0.12 & -0.04 & -0.08 \\
\hline Real GDP Growth & 5. 55 & 5. 35 & 0.10 & 5. 41 & 5. 28 & 0.13 & 5. 41 & 5. 10 & 0.31 & 4. 86 & 5. 10 & -0.24 \\
\hline Saving Rate & 13. 93 & 23. 67 & $-9.74 * * *$ & 13. 17 & 21. 35 & $-8.18 * * *$ & 12. 90 & 20.31 & $-7.41 * * *$ & 10.75 & 19. 59 & $-8.84 * * *$ \\
\hline Investment Rate & 23. 37 & 22. 41 & 0.97 & 24. 33 & 21.72 & 2. $61 * *$ & 24. 76 & 21.85 & 2. $9^{* *}$ & 24.18 & 21.63 & 2. $55^{* *}$ \\
\hline GDP per capita & 7215 & 9382 & -2167 & 7442 & 8811 & -1369 & 7807 & 8287 & -481 & 7057 & 8039 & -983 \\
\hline Return differential & 0.38 & -1.37 & 1. $75^{* * *}$ & 0.63 & -1.07 & 1. $7^{* * *}$ & 1. 49 & -0.94 & 2. $42 * * *$ & 2. 40 & -0.86 & 3. $27^{* * *}$ \\
\hline Net official flows to CA & 34.4 & 19.5 & 0.00 & 28.8 & 25 & 3. 8 & 27.3 & 28.3 & -1.0 & 25.1 & 29.8 & -4.7 \\
\hline Net official flows to GDP & 2.7 & 0.6 & 2. $1^{* * *}$ & 2.9 & 0.9 & 2. $0^{* * *}$ & 3. 1 & 1.1 & 2. $0^{* * *}$ & 3. 2 & 1. 3 & 1. $9 * * *$ \\
\hline External debt to GDP & 74. 22 & 44.80 & 29. $42 * * *$ & 83. 03 & 47. 30 & $35.73^{* * *}$ & 92.44 & 51.74 & $40.7^{* * *}$ & 100.86 & 54.27 & 46. $59 * * *$ \\
\hline Short Term Debt to Ext Debt & 13. 69 & 14. 04 & -0.35 & 13. 26 & 14. 23 & -0.97 & 13. 45 & 13. 61 & -0.17 & 14. 13 & 13. 17 & 0.96 \\
\hline Pub. Ext Debt to Ext Debt & 91.55 & 85. 16 & 6. $39 * *$ & 92.50 & 86. 72 & 5. $78^{* *}$ & 94.17 & 88. 06 & 6. $11^{* *}$ & 95.28 & 88. 23 & 7. $05 * *$ \\
\hline Concessional debt to Ext Debt & 30.99 & 16. 08 & 14. $91^{* * *}$ & 32.00 & 18. 25 & 13. $75^{* * *}$ & 31. 37 & 21.16 & $10.2 * *$ & 28.77 & 22. 79 & 5. 98 \\
\hline
\end{tabular}




\section{Table 4: Regional distribution of episodes controlling for GDP and GDP per capita}

This table reports the results of a set of probit regression where the dependent variable takes a value 1 during episodes and 0 in control periods. The control variables are dummy variables for Sub-Saharan Africa (SSA), Asia (ASIA), Middle East and North Africa (MNA), Latin America and the Caribbean (LAC), and emerging Europe (EME). The excluded group is advanced economies. The remaining controls are the log of GDP and log of GDP per capita (both measured in constant dollars).

\begin{tabular}{lcccccccc}
\hline & $(1)$ & $(2)$ & $(3)$ & $(4)$ & $(5)$ & $(6)$ & $(7)$ & $(8)$ \\
\hline Ln(GDP) & & $-0.137 * * *$ & & $-0.093^{* * *}$ & & $-0.049^{* * *}$ & $(0.011)$ & $-0.001^{* *}$ \\
& & $(0.025)$ & & $(0.015)$ & & 0.001 & $(0.0005)$ \\
Ln(GDPPC) & & $-0.070^{*}$ & & -0.021 & & 0.0003 \\
& & $(0.037)$ & & $(0.022)$ & & $(0.012)$ & $(0.0003)$ \\
SSA & $0.398^{* * *}$ & $-0.334^{* * *}$ & $0.315^{* * *}$ & $-0.168^{* * *}$ & $0.288^{* * *}$ & -0.0470 & $0.895^{* * *}$ & $0.736^{* * *}$ \\
& $(0.087)$ & $(0.090)$ & $(0.092)$ & $(0.053)$ & $(0.092)$ & $(0.039)$ & $(0.038)$ & $(0.115)$ \\
ASIA & 0.121 & $-0.300^{* * *}$ & 0.026 & $-0.158^{* * *}$ & -0.030 & $-0.078^{* * *}$ & & \\
& $(0.112)$ & $(0.079)$ & $(0.098)$ & $(0.035)$ & $(0.069)$ & $(0.024)$ & & \\
MNA & 0.086 & $-0.240^{* * *}$ & 0.042 & $-0.133^{* * *}$ & 0.036 & $-0.051^{* *}$ & $0.924^{* * *}$ & $0.906^{* * *}$ \\
& $(0.130)$ & $(0.085)$ & $(0.103)$ & $(0.036)$ & $(0.089)$ & $(0.024)$ & $(0.022)$ & $(0.087)$ \\
LAC & 0.127 & $-0.342^{* * *}$ & 0.063 & $-0.179^{* * *}$ & 0.075 & $-0.064^{* *}$ & $0.795^{* * *}$ & $0.702^{* * *}$ \\
& $(0.096)$ & $(0.074)$ & $(0.083)$ & $(0.044)$ & $(0.074)$ & $(0.032)$ & $(0.079)$ & $(0.125)$ \\
EME & $0.467 * * *$ & 0.041 & $0.383^{* * *}$ & 0.008 & $0.352^{* *}$ & 0.094 & $0.984^{* * *}$ & $0.990^{* * *}$ \\
& $(0.105)$ & $(0.153)$ & $(0.131)$ & $(0.093)$ & $(0.138)$ & $(0.096)$ & $(0.014)$ & $(0.005)$ \\
\hline Observations & 250 & 247 & 278 & 275 & 304 & 299 & 280 & 274 \\
\hline Sample & All & All & All & All & All & All & All & All \\
Threshold & $4 \%$ & $4 \%$ & $6 \%$ & $6 \%$ & $8 \%$ & $8 \%$ & $10 \%$ & $10 \%$ \\
\hline
\end{tabular}

Robust standard errors in parentheses

$* * * \mathrm{p}<0.01, * * \mathrm{p}<0.05, * \mathrm{p}<0.1$

\section{Table 5: Correlates of current account episodes (capital account openness, terms of trade and} savings rate)

This table reports the results of a set of probit regression where the dependent variable takes a value 1 during episodes and 0 in control periods. The control variables are the log of GDP and log of GDP per capita (both measured in constant dollars), the Chinn and Ito index of capital account openness, national savings over GDP, and terms of trade.

\begin{tabular}{|c|c|c|c|c|c|c|c|c|}
\hline & (1) & $(2)$ & (3) & (4) & (5) & (6) & (7) & (8) \\
\hline Ln(GDP) & $\begin{array}{c}-0.097 * * * \\
(0.024)\end{array}$ & $\begin{array}{c}-0.121^{* * *} \\
(0.031)\end{array}$ & $\begin{array}{c}-0.068 * * * \\
(0.015)\end{array}$ & $\begin{array}{c}-0.083^{* * *} \\
(0.020)\end{array}$ & $\begin{array}{c}-0.044^{* * *} \\
(0.010)\end{array}$ & $\begin{array}{c}-0.065^{* * *} \\
(0.016)\end{array}$ & $\begin{array}{c}-0.015 * * \\
(0.006)\end{array}$ & $\begin{array}{c}-0.030 * * \\
(0.012)\end{array}$ \\
\hline Ln(GDPPC) & $\begin{array}{c}0.009 \\
(0.033)\end{array}$ & $\begin{array}{l}-0.011 \\
(0.049)\end{array}$ & $\begin{array}{c}0.025 \\
(0.019)\end{array}$ & $\begin{array}{c}0.011 \\
(0.027)\end{array}$ & $\begin{array}{c}0.011 \\
(0.012)\end{array}$ & $\begin{array}{c}0.018 \\
(0.019)\end{array}$ & $\begin{array}{c}0.004 \\
(0.005)\end{array}$ & $\begin{array}{l}0.0120 \\
(0.012)\end{array}$ \\
\hline CA Open & $\begin{array}{l}0.211^{*} \\
(0.115)\end{array}$ & $\begin{array}{c}0.217 \\
(0.149)\end{array}$ & $\begin{array}{l}0.124^{*} \\
(0.069)\end{array}$ & $\begin{array}{c}0.128 \\
(0.087)\end{array}$ & $\begin{array}{c}0.090 * * \\
(0.045)\end{array}$ & $\begin{array}{c}0.088 \\
(0.063)\end{array}$ & $\begin{array}{c}0.013 \\
(0.018)\end{array}$ & $\begin{array}{c}0.029 \\
(0.039)\end{array}$ \\
\hline Saving rate & $\begin{array}{c}-0.026 * * * \\
(0.005)\end{array}$ & $\begin{array}{c}-0.023^{* * *} \\
(0.006)\end{array}$ & $\begin{array}{c}-0.011^{* * *} \\
(0.003)\end{array}$ & $\begin{array}{c}-0.012^{* * *} \\
(0.004)\end{array}$ & $\begin{array}{c}-0.006^{* * *} \\
(0.002)\end{array}$ & $\begin{array}{c}-0.007^{* * *} \\
(0.003)\end{array}$ & $\begin{array}{l}-0.002 * \\
(0.001)\end{array}$ & $\begin{array}{c}-0.005^{* *} \\
(0.002)\end{array}$ \\
\hline Ter. of tr. & $\begin{array}{c}-0.287 * * \\
(0.131)\end{array}$ & $\begin{array}{c}-0.328 * * \\
(0.145)\end{array}$ & $\begin{array}{c}-0.112^{*} \\
(0.066)\end{array}$ & $\begin{array}{c}-0.146^{*} \\
(0.080)\end{array}$ & $\begin{array}{c}-0.076 * * \\
(0.038)\end{array}$ & $\begin{array}{l}-0.100^{*} \\
(0.051)\end{array}$ & $\begin{array}{l}-0.022 \\
(0.016)\end{array}$ & $\begin{array}{l}-0.046 \\
(0.031)\end{array}$ \\
\hline N. Obs. & 205 & 139 & 230 & 162 & 247 & 175 & 253 & 181 \\
\hline Sample & All & Dev & All & Dev & All & Dev & All & Dev \\
\hline Threshold & $4 \%$ & $4 \%$ & $6 \%$ & $6 \%$ & $8 \%$ & $8 \%$ & $10 \%$ & $10 \%$ \\
\hline
\end{tabular}

Robust standard errors in parentheses

*** $\mathrm{p}<0.01, * * \mathrm{p}<0.05,{ }^{*} \mathrm{p}<0.1$ 
Table 6: Correlates of current account episodes (FDI and portfolio inflows).

This table reports the results of a set of probit regression where the dependent variable takes a value 1 during episodes and 0 in control periods. The control variables are the log of GDP and log of GDP per capita (both measured in constant dollars), the Chinn and Ito index of capital account openness, national savings over GDP, terms of trade, official flows over GDP, FDI flows over GDP and portfolio flows over GDP.

\begin{tabular}{lcccccc}
\hline & $(1)$ & $(2)$ & $(3)$ & $(4)$ & $(5)$ & $(6)$ \\
\hline Ln(GDP) & $-0.064^{* * *}$ & -0.056 & $-0.039^{* * *}$ & $-0.033^{*}$ & $-0.024^{* * *}$ & -0.014 \\
& $(0.024)$ & $(0.035)$ & $(0.013)$ & $(0.019)$ & $(0.009)$ & $(0.014)$ \\
Ln(GDPPC) & 0.038 & 0.0437 & $0.043^{* *}$ & $0.052^{*}$ & $0.026^{* *}$ & 0.027 \\
& $(0.038)$ & $(0.060)$ & $(0.020)$ & $(0.030)$ & $(0.012)$ & $(0.019)$ \\
CA Open & $0.254^{* *}$ & $0.361^{* *}$ & $0.119^{*}$ & 0.134 & 0.064 & 0.035 \\
& $(0.120)$ & $(0.157)$ & $(0.0643)$ & $(0.082)$ & $(0.039)$ & $(0.036)$ \\
Saving rate & $-0.022^{* * *}$ & $-0.021^{* * *}$ & $-0.0105^{* * *}$ & $-0.011^{* * *}$ & $-0.005^{* *}$ & -0.003 \\
& $(0.006)$ & $(0.007)$ & $(0.003)$ & $(0.004)$ & $(0.002)$ & $(0.003)$ \\
Ter. of tr. & $-0.402^{* *}$ & $-0.500^{* * *}$ & -0.118 & -0.159 & -0.037 & -0.020 \\
& $(0.157)$ & $(0.192)$ & $(0.0840)$ & $(0.100)$ & $(0.038)$ & $(0.034)$ \\
Official & $0.100^{* *}$ & $0.129 * * *$ & $0.055^{* * *}$ & $0.069^{* * *}$ & $0.024^{* *}$ & 0.022 \\
& $(0.039)$ & $(0.048)$ & $(0.018)$ & $(0.025)$ & $(0.011)$ & $(0.015)$ \\
FDI & $0.003^{* * *}$ & $0.005^{* * *}$ & $0.0011^{* *}$ & $0.002^{* *}$ & 0.0003 & 0.0003 \\
& $(0.0009)$ & $(0.001)$ & $(0.0005)$ & $(0.0007)$ & $(0.0003)$ & $(0.0003)$ \\
Portfolio & $-0.003^{* *}$ & $-0.007 * * *$ & $-0.00138^{* *}$ & $-0.003^{* *}$ & $-0.0013^{* *}$ & $-0.003^{* * *}$ \\
& $(0.001)$ & $(0.002)$ & $(0.0006)$ & $(0.0015)$ & $(0.0006)$ & $(0.001)$ \\
\hline N. Obs & 196 & 132 & 216 & 150 & 232 & 160 \\
Sample & All & Dev & All & Dev & All & Dev \\
Threshold & $4 \%$ & $4 \%$ & $6 \%$ & $6 \%$ & $8 \%$ & $8 \%$ \\
\hline Robust & & & & & & 160 \\
\hline
\end{tabular}

Robust standard errors in parentheses

*** $\mathrm{p}<0.01,{ }^{* *} \mathrm{p}<0.05,{ }^{*} \mathrm{p}<0.1$

Table 7: Growth Volatility

This table reports the results of the GARCH estimates of the model described in Equation (4).

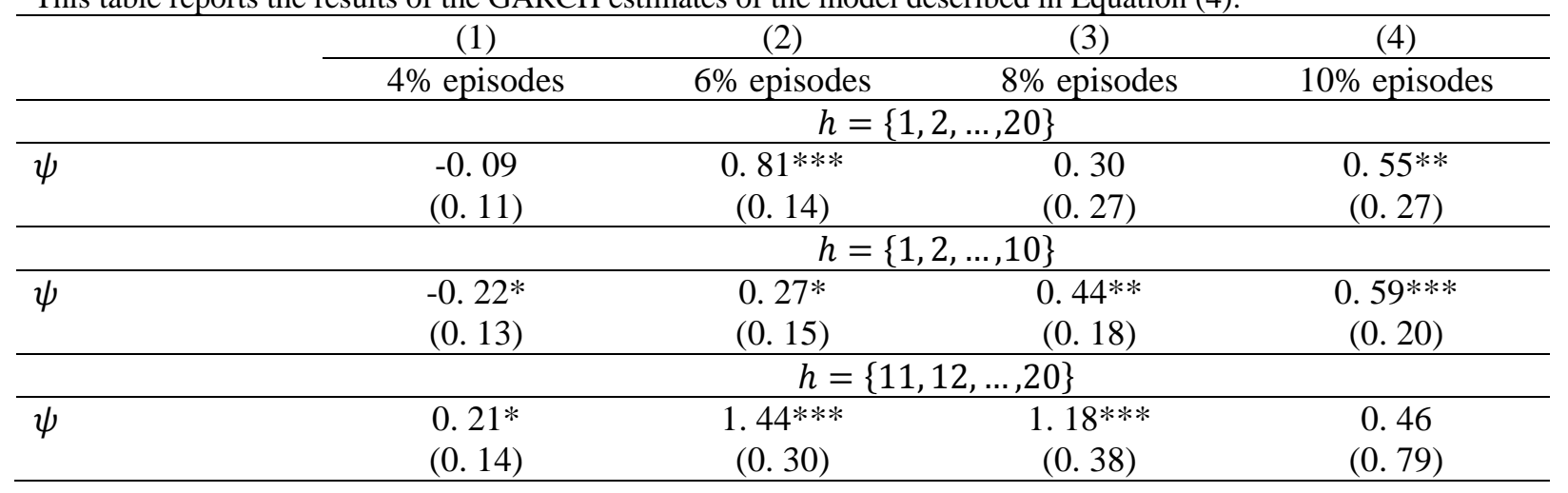


Figure 1: Evolution of main economic variables during episodes with $4 \%$ Threshold
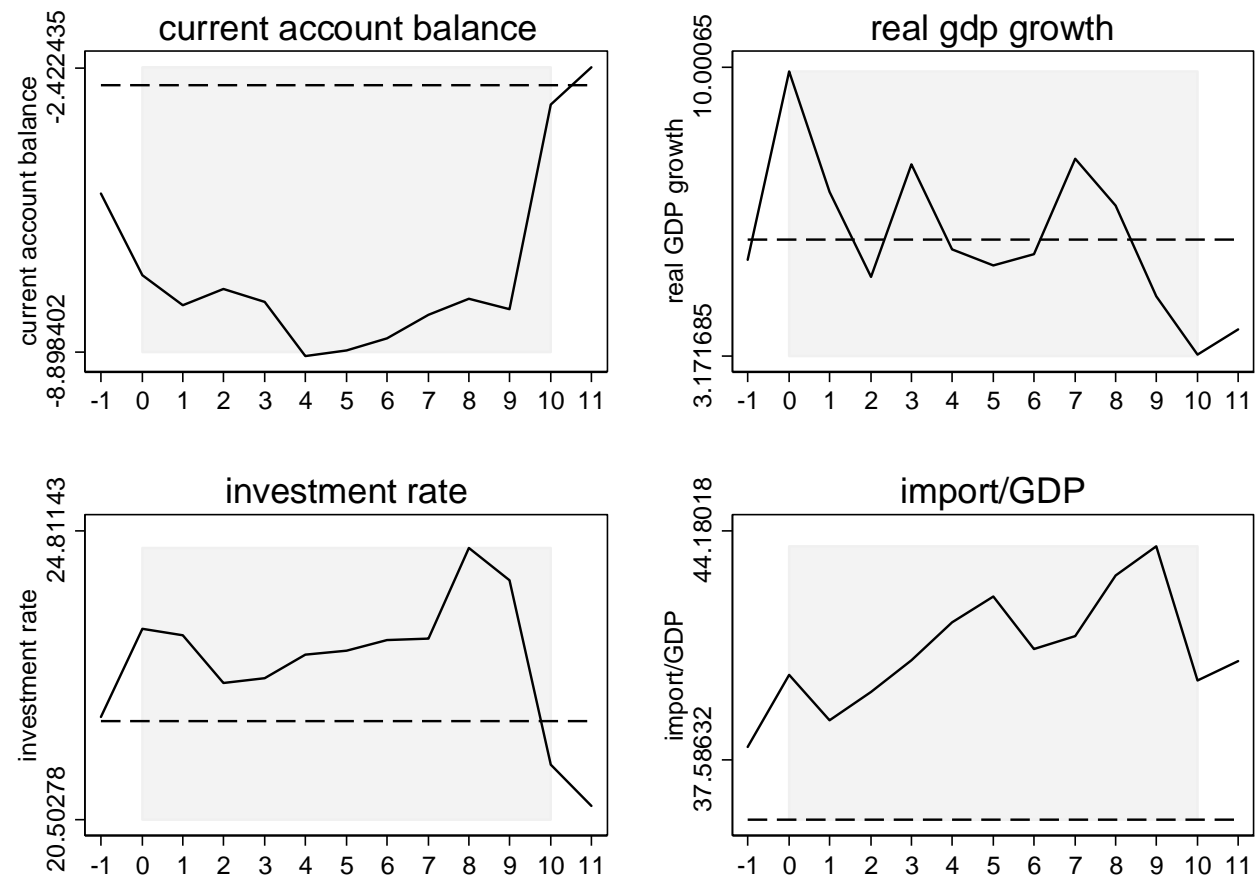
Figure 2: Evolution of main economic variables during episodes with 6\% Threshold
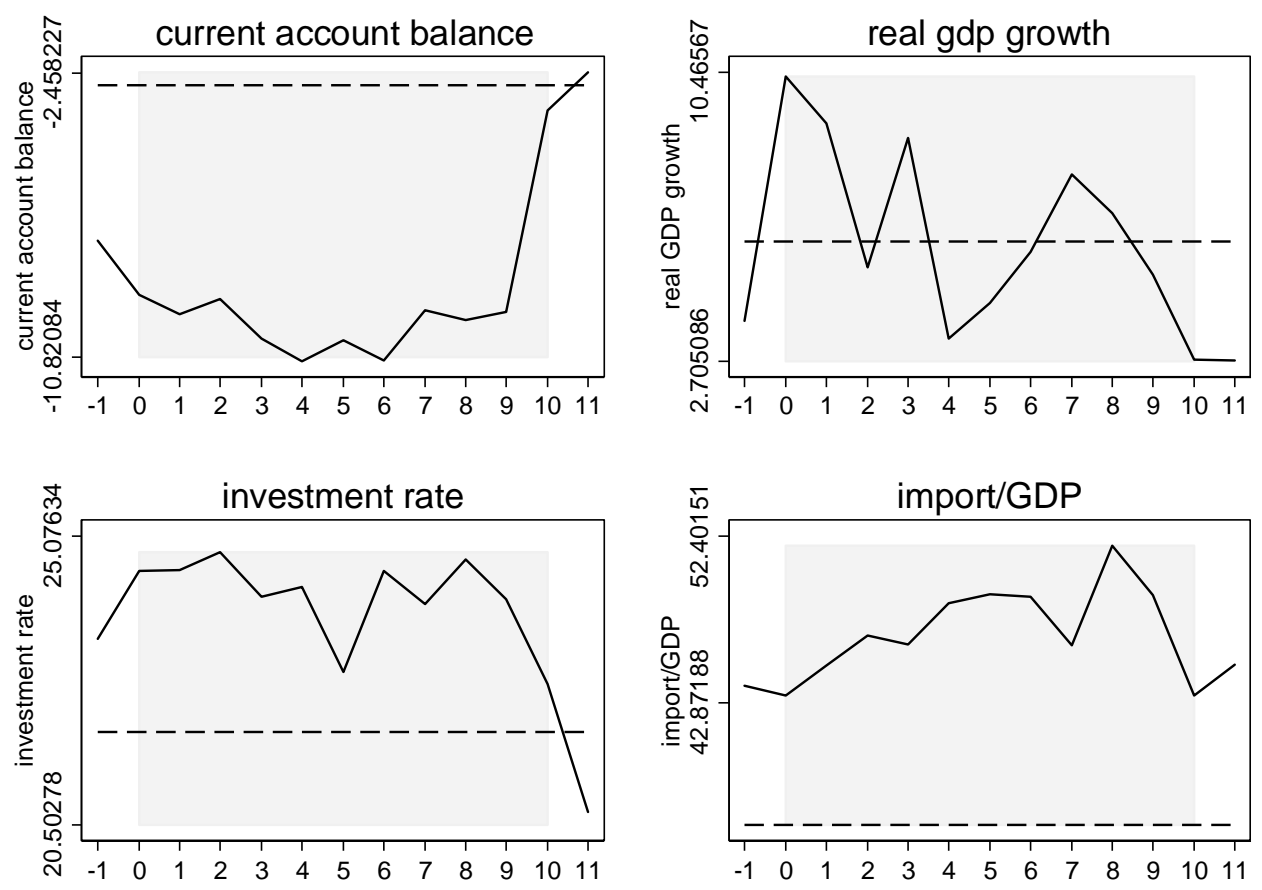
Figure 3: Evolution of main economic variables during episodes with $8 \%$ Threshold
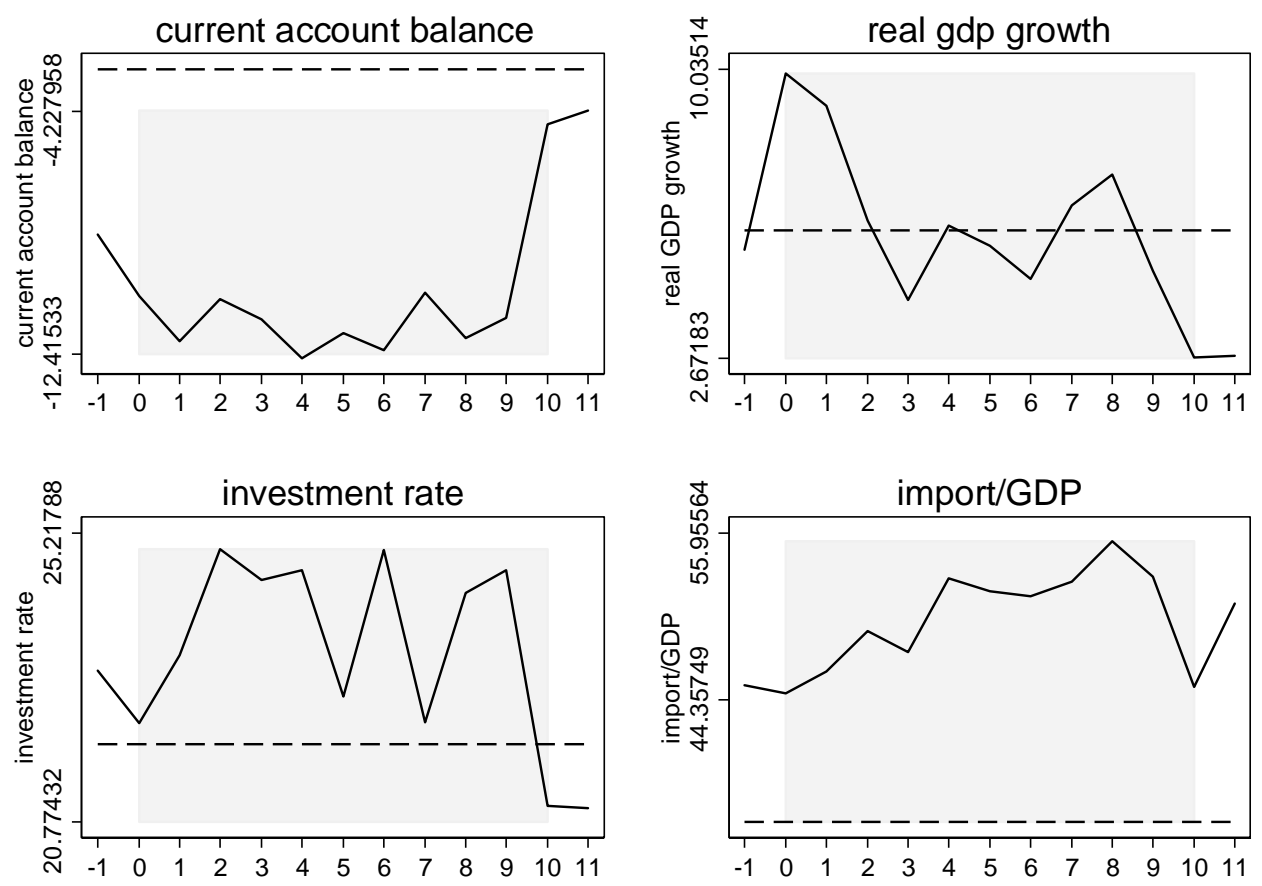
Figure 4: Evolution of main economic variables during episodes with $10 \%$ Threshold
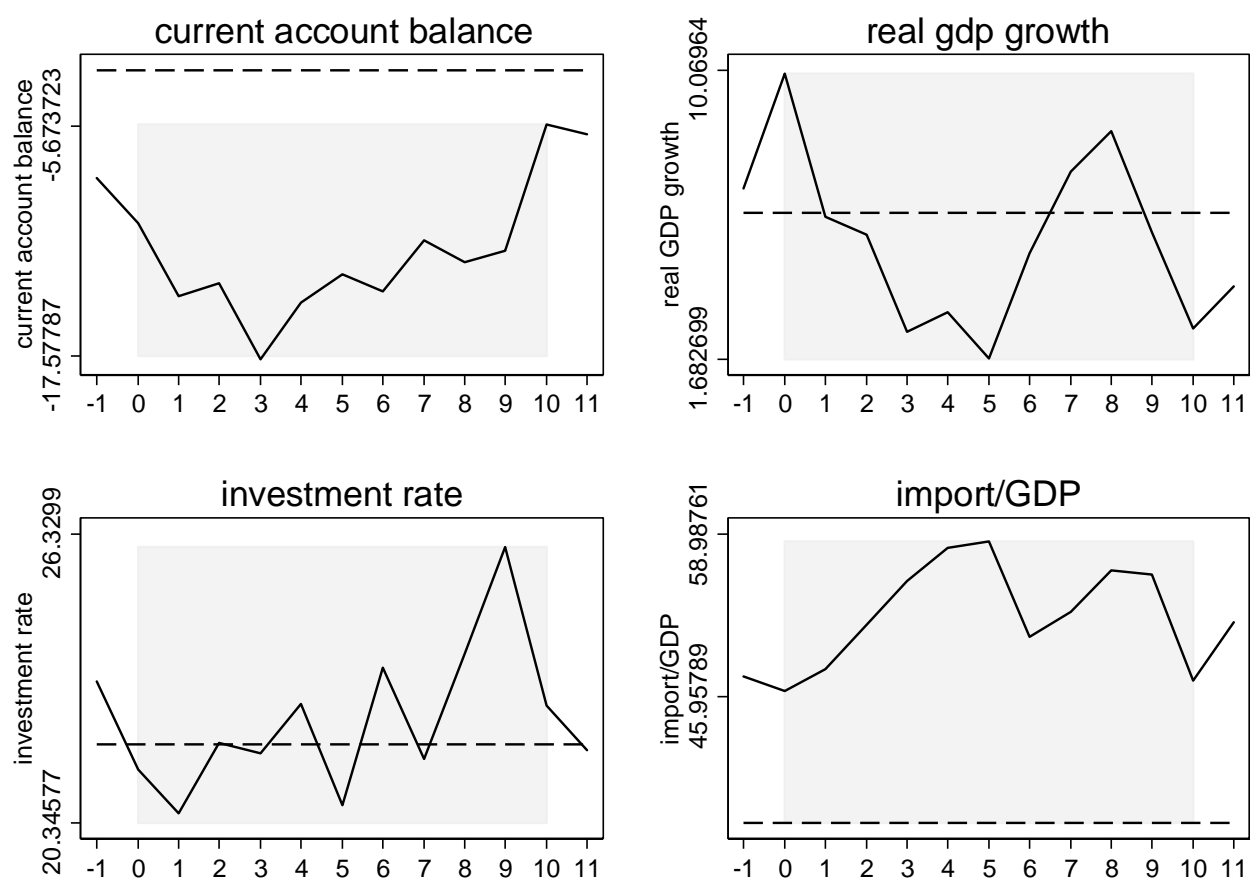
Figure 5: The Financing of Large and Persistent Current Account Deficits
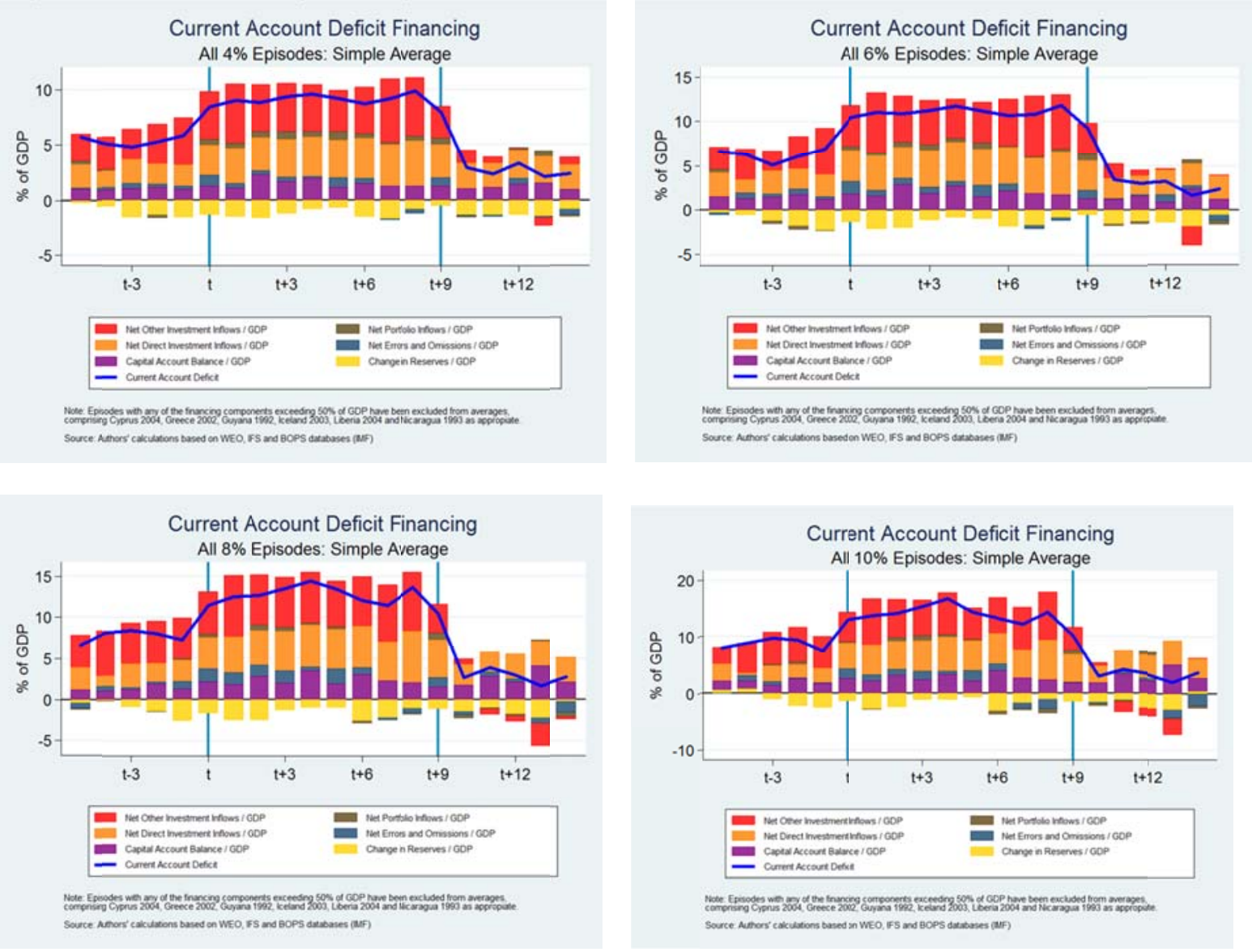
Figure 6: GDP growth after the inset of the episode, no controls

These figures are based on the estimates of Tables A8-A11
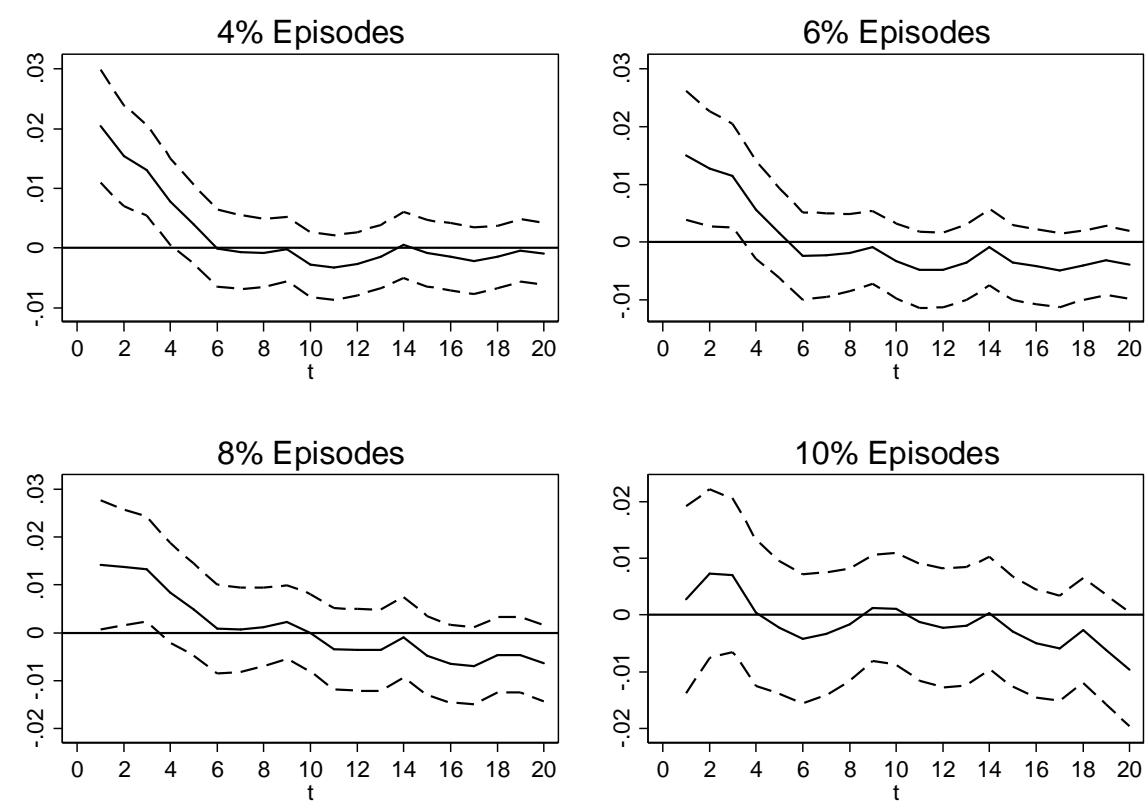

Figure 7: GDP growth after the inset of the episode controlling for convergence, human capital, and saving rate

These figures are based on the estimates of Tables A12-A15
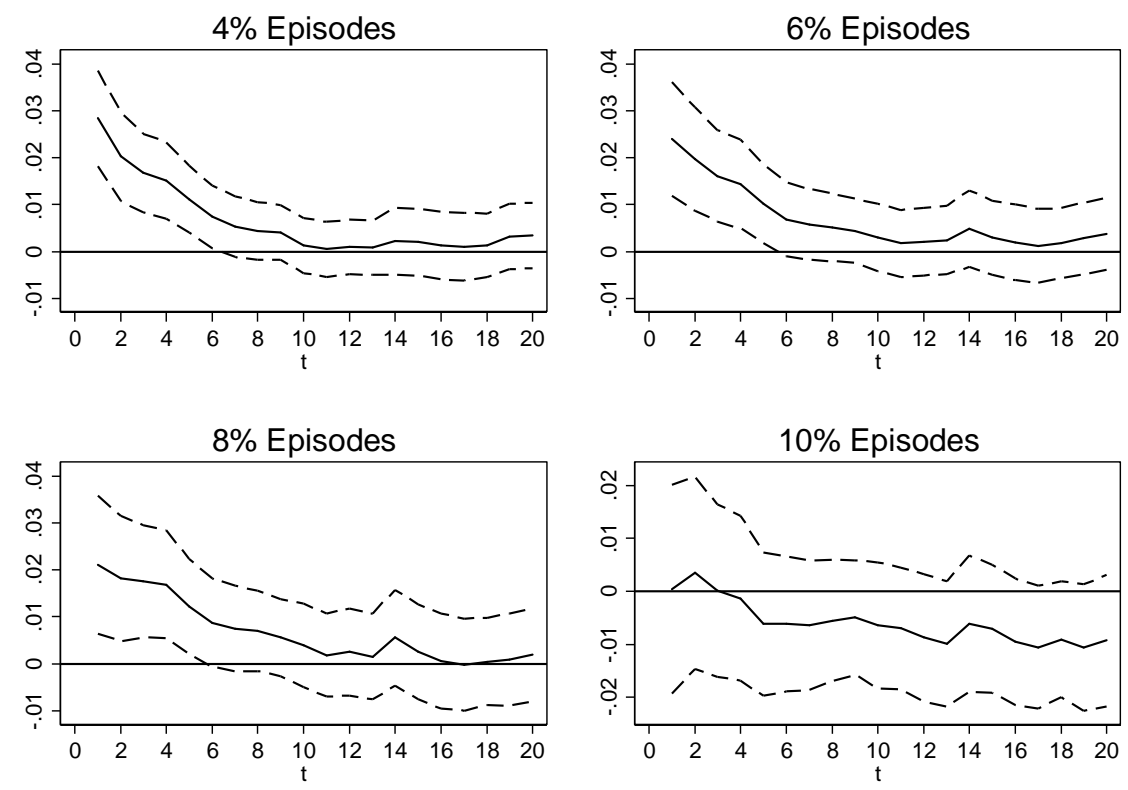
Figure 8: GDP growth after the inset of the episode controlling for convergence, human capital, and saving rate and for a differential effects in episodes financed by official flows (grey line)

These figures are based on the estimates of Tables A16-A19
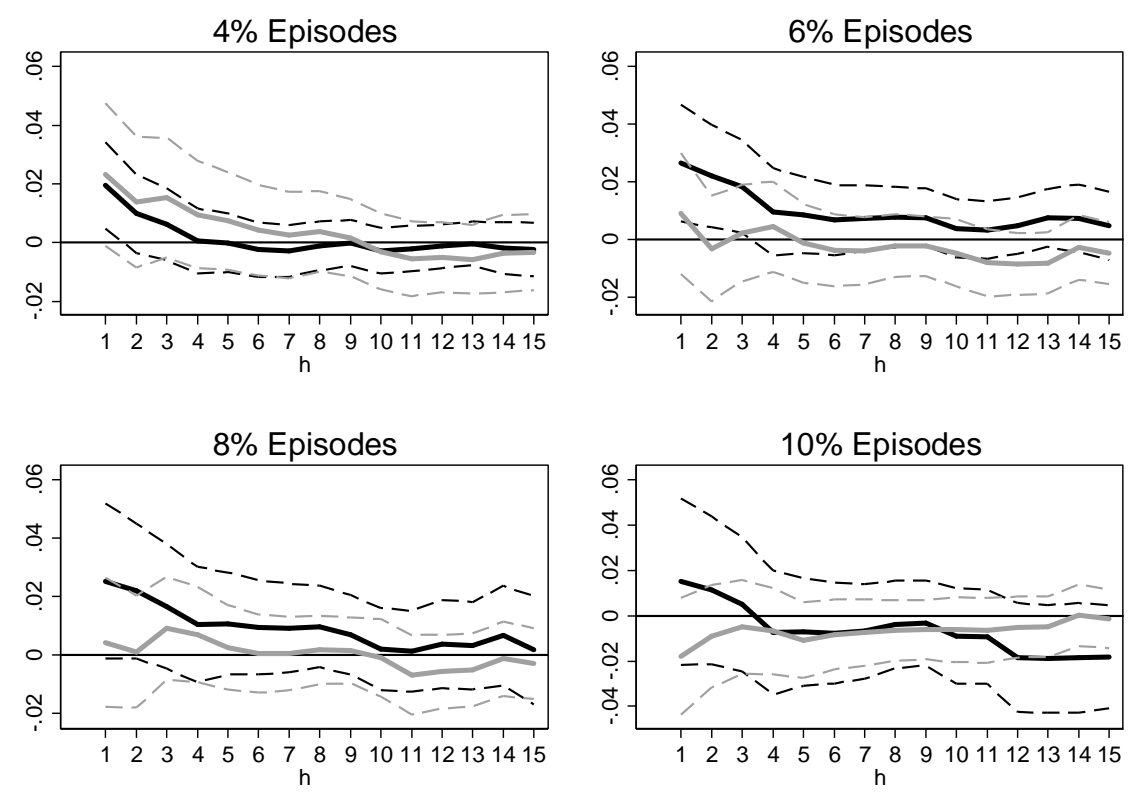

Figure 9: GDP growth after the inset of the episode controlling for convergence, human capital, and saving rate and for a differential effects in episodes with above median FDI inflows (grey line) These figures are based on the estimates of Tables A20-A23
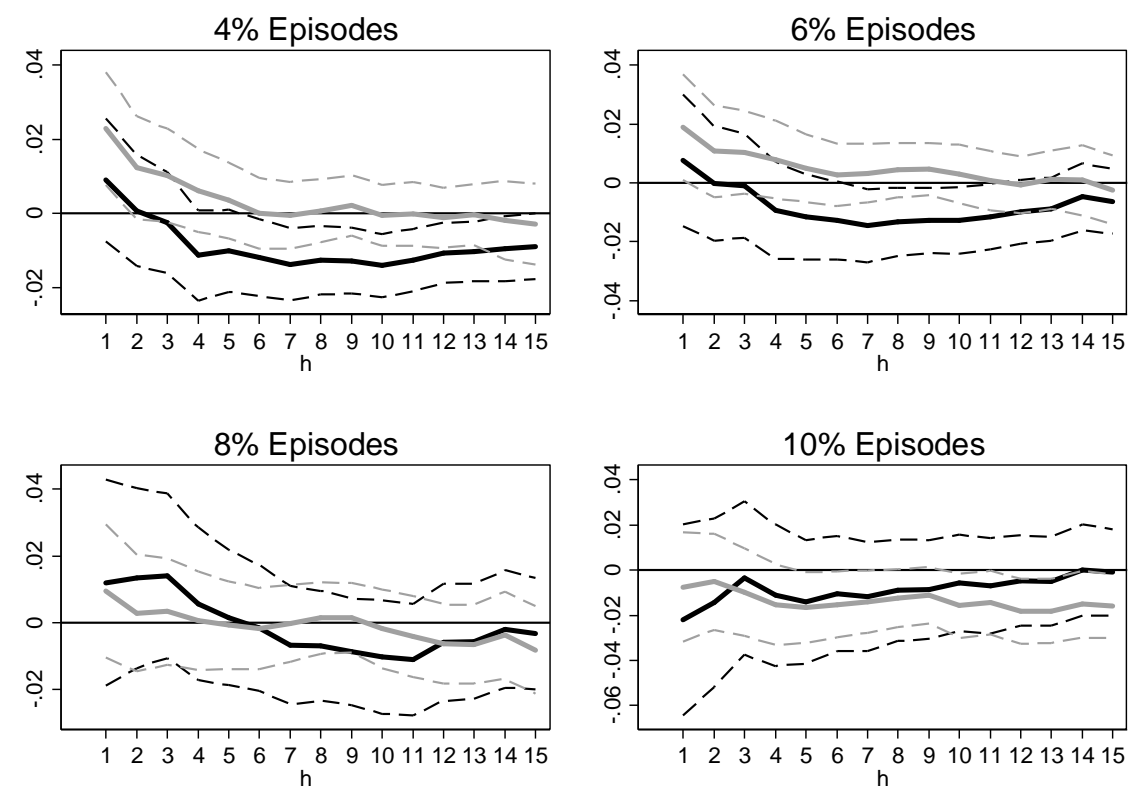
Figure 10: Counterfactual analysis: The level of income with and without episodes (controlling for convergence, human capital, and saving rate)

These figures are based on the estimates of Tables A12-A15
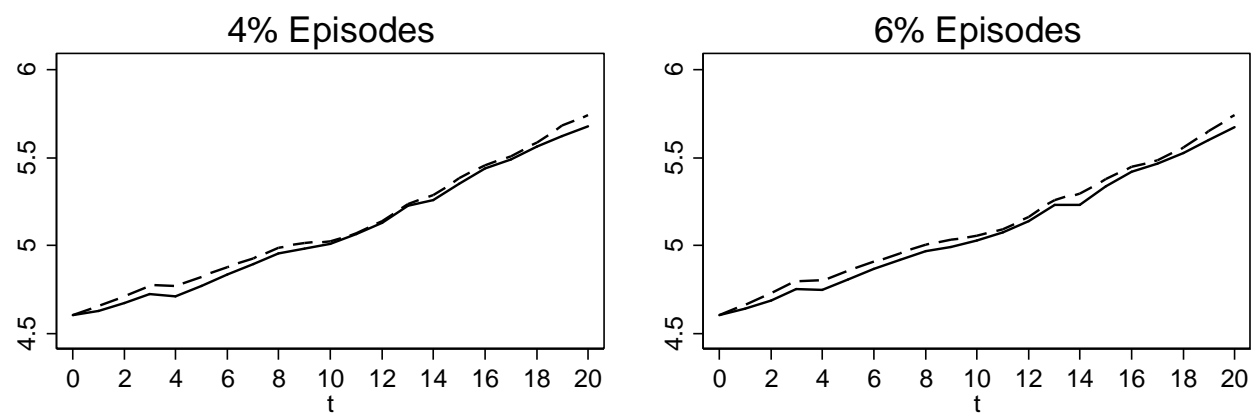

8\% Episodes

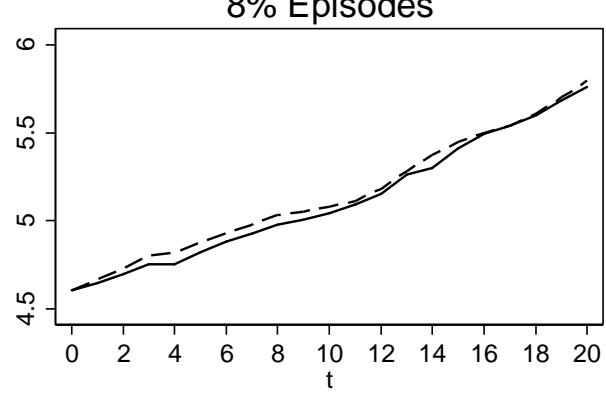

10\% Episodes

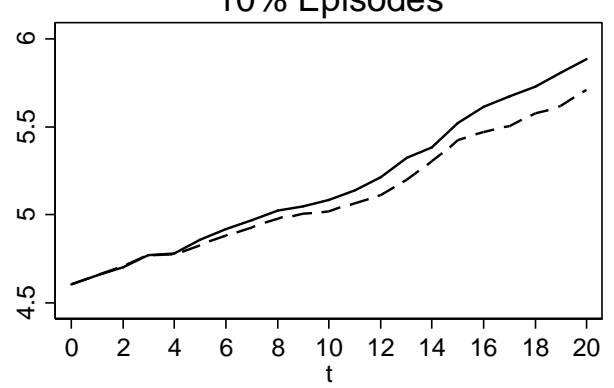


Figure 11: GDP growth after the inset of the episode controlling for convergence, human capital, and saving rate, Effect of episode identified using heteroskedasticity
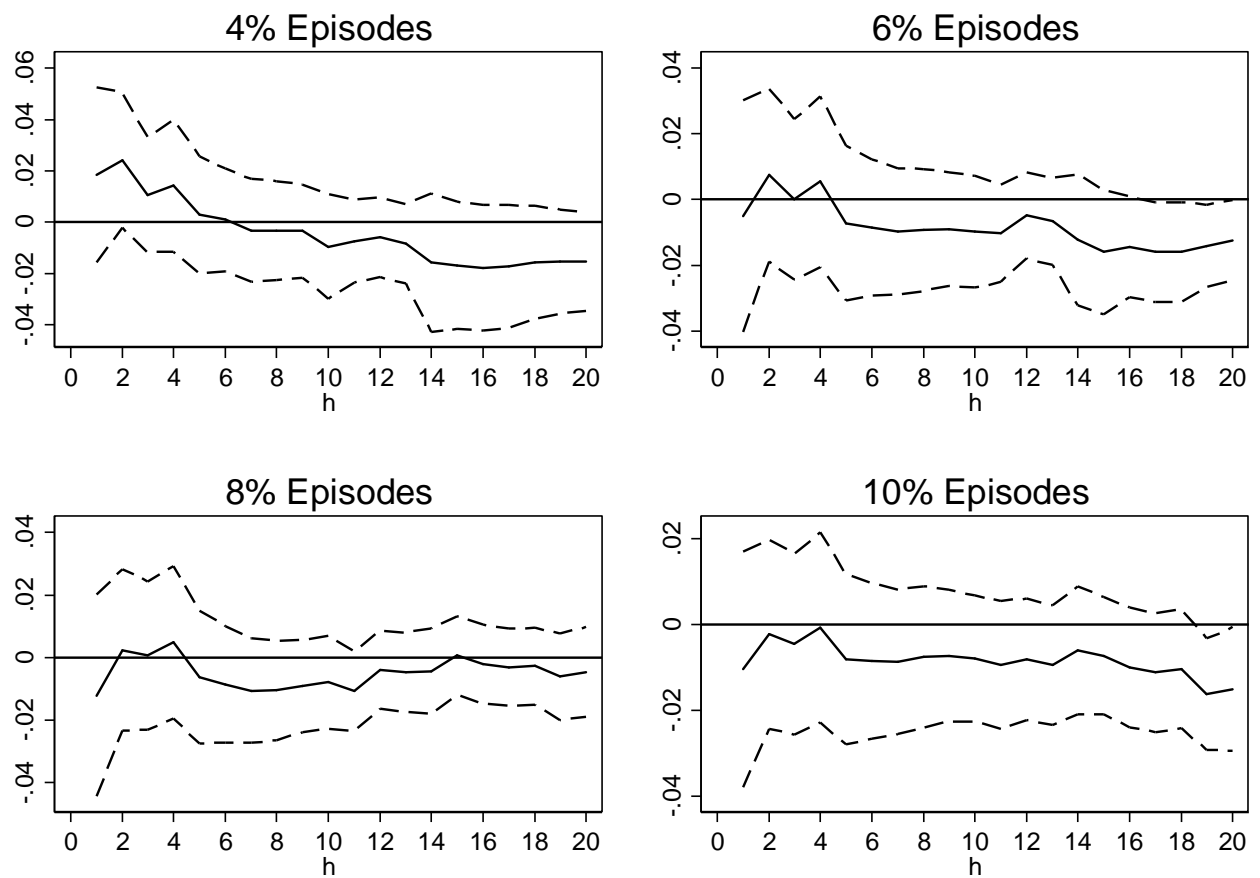
Figure 12: Counterfactual analysis: The level of income with and without episodes (controlling for convergence, human capital, and saving rate). Effect of episode identified using heteroskedasticity
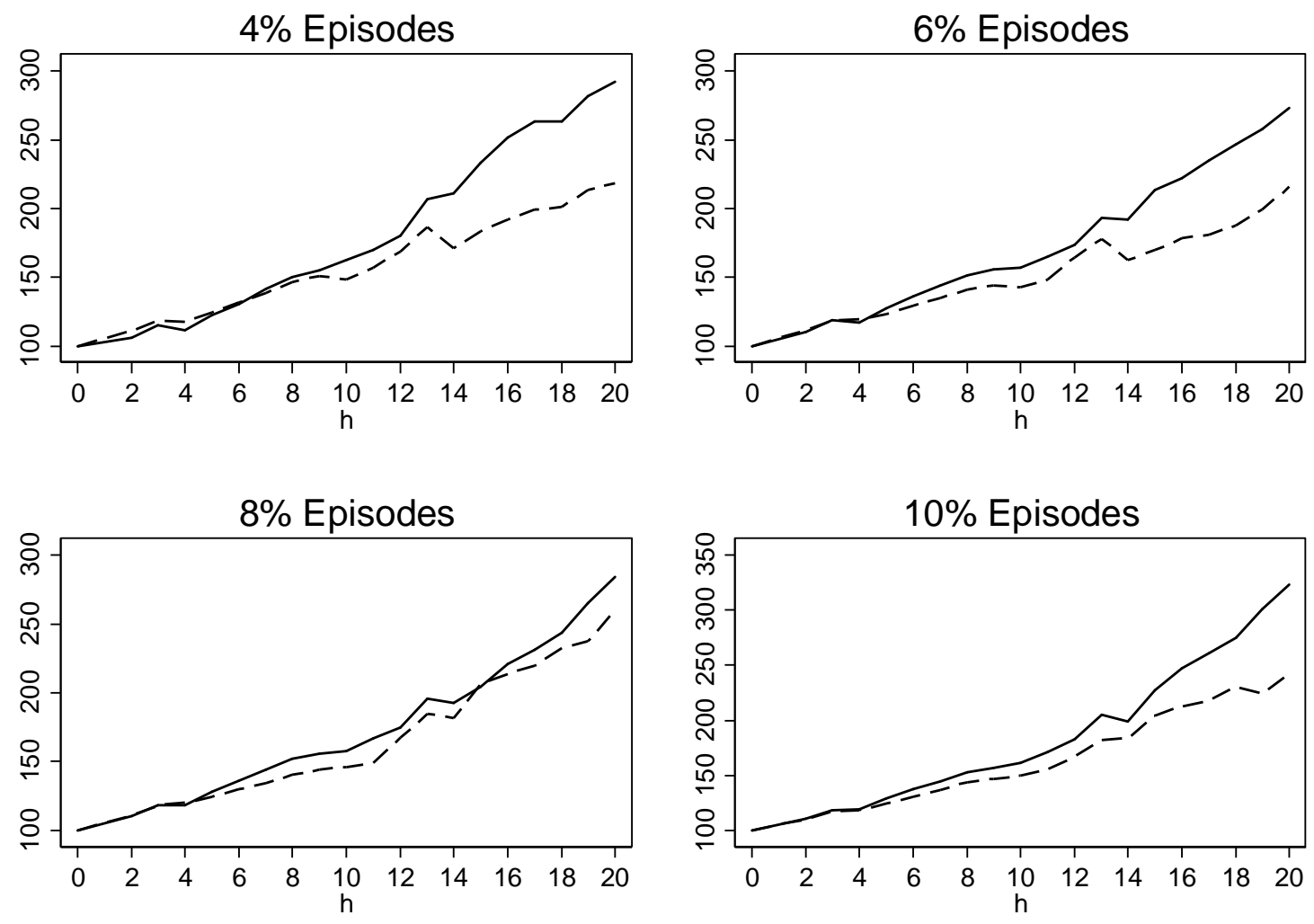


\section{Appendix}

Table A1: Full list of episodes at various current account deficit thresholds

\begin{tabular}{|c|c|c|c|c|c|c|c|c|c|c|c|c|c|}
\hline \multicolumn{5}{|c|}{$4 \%$} & \multicolumn{5}{|c|}{$6 \%$} & \multicolumn{2}{|l|}{$8 \%$} & \multicolumn{2}{|c|}{$10 \%$} \\
\hline AUS1981 & $\mathrm{AE}$ & ALB2004 & EME & BEN1979 & SSA & CYP1976 & $\mathrm{AE}$ & COG1987 & SSA & GRC2002 & $\mathrm{AE}$ & LBN2002 & MNA \\
\hline AUS2003 & $\mathrm{AE}$ & ARM1994 & EME & BEN1997 & SSA & ESP2001 & $\mathrm{AE}$ & CPV2003 & SSA & PRT2000 & $\mathrm{AE}$ & ARM1994 & EME \\
\hline CYP1976 & $\mathrm{AE}$ & BGR2000 & EME & CAF1983 & SSA & GRC2002 & $\mathrm{AE}$ & GHA2004 & SSA & SGP1973 & $\mathrm{AE}$ & BIH1999 & EME \\
\hline CYP2004 & $\mathrm{AE}$ & BIH1999 & EME & CMR1978 & SSA & IRL1977 & $\mathrm{AE}$ & GNB1987 & SSA & MDV2003 & Asia & EST1999 & EME \\
\hline ESP2001 & $\mathrm{AE}$ & CZE1995 & EME & COG1987 & SSA & NZL1979 & $\mathrm{AE}$ & LBR2004 & SSA & LBN2002 & MNA & GEO2003 & EME \\
\hline GRC1977 & $\mathrm{AE}$ & EST1999 & EME & CPV2003 & SSA & PRT2000 & $\mathrm{AE}$ & LSO1995 & SSA & ALB2004 & EME & BHS2004 & LAC \\
\hline GRC2002 & $\mathrm{AE}$ & GEO2003 & EME & GHA1992 & SSA & SGP1974 & $\mathrm{AE}$ & MDG1979 & SSA & ARM1994 & EME & BRB1970 & LAC \\
\hline IRL1977 & $\mathrm{AE}$ & HRV1997 & EME & GHA2004 & SSA & MDV2003 & Asia & MDG1990 & SSA & BIH1999 & EME & JAM2002 & LAC \\
\hline ISL2003 & $\mathrm{AE}$ & HUN1998 & EME & GNB1987 & SSA & NPL1988 & Asia & MLI1981 & SSA & EST1999 & EME & NIC1993 & LAC \\
\hline NZL1979 & $\mathrm{AE}$ & LTU1999 & EME & KEN1990 & SSA & PNG1980 & Asia & MOZ2004 & SSA & GEO2003 & EME & COG1990 & SSA \\
\hline NZL1999 & $\mathrm{AE}$ & LVA1999 & EME & LBR2004 & SSA & VUT1987 & Asia & MRT1979 & SSA & LTU1999 & EME & CPV1995 & SSA \\
\hline PRT2000 & $\mathrm{AE}$ & ROU2003 & EME & LSO1995 & SSA & LBN2002 & MNA & MWI1979 & SSA & LVA1999 & EME & GNB1987 & SSA \\
\hline SGP1975 & $\mathrm{AE}$ & BHS2004 & LAC & MDG1979 & SSA & SAU1983 & MNA & NER1986 & SSA & BHS2004 & LAC & LBR2004 & SSA \\
\hline USA1999 & $\mathrm{AE}$ & BLZ1999 & LAC & MDG1990 & SSA & TUN1977 & MNA & SDN2000 & SSA & BRB1970 & LAC & LSO1995 & SSA \\
\hline KHM2004 & Asia & BOL1984 & LAC & MLI1981 & SSA & ALB2004 & EME & SEN1979 & SSA & GUY1992 & LAC & MLI1981 & SSA \\
\hline LAO1988 & Asia & BRB1970 & LAC & MOZ2004 & SSA & ARM1994 & EME & SEN2002 & SSA & HND1976 & LAC & MOZ2004 & SSA \\
\hline LKA1985 & Asia & CHL1978 & LAC & MRT1979 & SSA & BIH1999 & EME & SLE2003 & SSA & JAM2002 & LAC & MRT1979 & SSA \\
\hline MDV2003 & Asia & CRI1999 & LAC & MWI1979 & SSA & EST1999 & EME & SYC1980 & SSA & NIC1993 & LAC & SEN1979 & SSA \\
\hline NPL1988 & Asia & DOM1974 & LAC & NER1986 & SSA & GEO2003 & EME & SYC2004 & SSA & COG1990 & SSA & SLE2003 & SSA \\
\hline PAK1988 & Asia & GTM1998 & LAC & RWA1980 & SSA & HUN1998 & EME & TG01997 & SSA & CPV2003 & SSA & SYC1980 & SSA \\
\hline PNG1980 & Asia & GUY1992 & LAC & SDN2000 & SSA & LTU1999 & EME & TZA1990 & SSA & GHA2004 & SSA & SYC2004 & SSA \\
\hline THA1976 & Asia & GUY2004 & LAC & SEN1979 & SSA & LVA1999 & EME & TZA2004 & SSA & GNB1987 & SSA & TGO1997 & SSA \\
\hline THA1988 & Asia & HND1979 & LAC & SEN2002 & SSA & ROU2003 & EME & ZMB1981 & SSA & LBR2004 & SSA & TZA1990 & SSA \\
\hline VUT1987 & Asia & HND2003 & LAC & SLE2003 & SSA & BHS2004 & LAC & & & LSO1995 & SSA & ZMB1981 & SSA \\
\hline ISR1975 & MNA & HTI1977 & LAC & SYC1980 & SSA & BRB1970 & LAC & & & MDG1979 & SSA & & \\
\hline LBN2002 & MNA & JAM2002 & LAC & SYC2004 & SSA & CHL1978 & LAC & & & MLI1981 & SSA & & \\
\hline SAU1983 & MNA & NIC1993 & LAC & TGO1997 & SSA & GUY1992 & LAC & & & MOZ2004 & SSA & & \\
\hline TUN1977 & MNA & PER1986 & LAC & TZA1990 & SSA & HND1978 & LAC & & & MRT1979 & SSA & & \\
\hline TUN1992 & MNA & PRY1979 & LAC & TZA2004 & SSA & HND2003 & LAC & & & NER1986 & SSA & & \\
\hline & & & & UGA1994 & SSA & JAM2002 & LAC & & & SEN1979 & SSA & & \\
\hline & & & & ZMB1981 & SSA & NIC1993 & LAC & & & SEN2002 & SSA & & \\
\hline & & & & & & PRY1979 & LAC & & & SLE2003 & SSA & & \\
\hline & & & & & & & & & & SYC1980 & SSA & & \\
\hline & & & & & & & & & & SYC2004 & SSA & & \\
\hline & & & & & & & & & & TG01997 & SSA & & \\
\hline & & & & & & & & & & TZA1990 & SSA & & \\
\hline & & & & & & & & & & ZMB1981 & SSA & & \\
\hline
\end{tabular}


Table A2: Correlates of current account episodes (return differentials)

\begin{tabular}{lcccccc}
\hline & $(1)$ & $(2)$ & $(3)$ & $(4)$ & $(5)$ & $(6)$ \\
\hline Ln(GDP) & $-0.0778^{* * *}$ & -0.0589 & $-0.0362^{* * *}$ & -0.0221 & $-0.0245^{* *}$ & -0.00663 \\
Ln(GDPPC) & $(0.0263)$ & $(0.0367)$ & $(0.0121)$ & $(0.0199)$ & $(0.0103)$ & $(0.0112)$ \\
& $0.0866^{* *}$ & $0.154^{* *}$ & $0.0422^{* *}$ & $0.0753^{* *}$ & $0.0304^{* *}$ & 0.0229 \\
CA Open & $(0.0364)$ & $(0.0602)$ & $(0.0182)$ & $(0.0321)$ & $(0.0126)$ & $(0.0262)$ \\
& $0.352^{* * *}$ & $0.533^{* * *}$ & $0.151^{* *}$ & $0.168^{*}$ & 0.0691 & 0.0280 \\
Saving rate & $(0.121)$ & $(0.172)$ & $(0.0687)$ & $(0.0927)$ & $(0.0429)$ & $(0.0382)$ \\
& $-0.0305^{* * *}$ & $-0.0375^{* * *}$ & $-0.00963^{* * *}$ & $-0.0125^{* * *}$ & $-0.00464^{* *}$ & -0.00261 \\
Tot & $(0.00692)$ & $(0.00934)$ & $(0.00292)$ & $(0.00415)$ & $(0.00215)$ & $(0.00300)$ \\
& $-0.779^{* *}$ & $-0.943^{* * *}$ & $-0.203^{*}$ & $-0.234^{*}$ & -0.0834 & -0.0385 \\
Off & $(0.317)$ & $(0.364)$ & $(0.109)$ & $(0.131)$ & $(0.0573)$ & $(0.0530)$ \\
& $0.164 * * *$ & $0.235^{* * *}$ & $0.0578^{* * *}$ & $0.0930^{* * *}$ & $0.0240^{*}$ & 0.0172 \\
FDI & $(0.0506)$ & $(0.0665)$ & $(0.0213)$ & $(0.0332)$ & $(0.0126)$ & $(0.0202)$ \\
& $0.00335^{* * *}$ & $0.00496^{* * *}$ & $0.00121^{* *}$ & $0.00152 *$ & 0.000307 & 0.000166 \\
PORT & $(0.00116)$ & $(0.00183)$ & $(0.000545)$ & $(0.000793)$ & $(0.000287)$ & $(0.000225)$ \\
& $-0.00375 * * *$ & $-0.0109^{* * *}$ & $-0.00167 * *$ & $-0.00378^{* *}$ & $-0.00142^{*}$ & $-0.00309 *$ \\
Ret. Diff. & $(0.00141)$ & $(0.00252)$ & $(0.000749)$ & $(0.00184)$ & $(0.000779)$ & $(0.00174)$ \\
& 0.0134 & 0.00737 & $0.0112^{*}$ & 0.0126 & $0.00873 *$ & 0.00513 \\
& $(0.0151)$ & $(0.0178)$ & $(0.00677)$ & $(0.00979)$ & $(0.00484)$ & $(0.00598)$ \\
\hline N. Obs. & 172 & 118 & 187 & 132 & 199 & 140 \\
Sample & All & Dev & All & Dev & All & Dev \\
Threshold & $4 \%$ & $4 \%$ & $6 \%$ & $6 \%$ & $8 \%$ & $8 \%$ \\
\hline
\end{tabular}

Robust standard errors in parentheses

*** $\mathrm{p}<0.01,{ }^{* *} \mathrm{p}<0.05,{ }^{*} \mathrm{p}<0.1$ 
Table A3: Correlates of current account episodes (imports)

\begin{tabular}{lcccccc}
\hline & $(1)$ & $(2)$ & $(3)$ & $(4)$ & $(5)$ & $(6)$ \\
\hline Ln(GDP) & $-0.0521^{*}$ & -0.0152 & -0.0136 & -0.00190 & -0.0105 & -0.00241 \\
Ln(GDPPC) & $(0.0315)$ & $(0.0419)$ & $(0.0141)$ & $(0.0228)$ & $(0.00794)$ & $(0.00723)$ \\
& $0.0687^{*}$ & $0.113^{*}$ & 0.0241 & $0.0522^{*}$ & $0.0174^{*}$ & 0.0172 \\
CA Open & $(0.0390)$ & $(0.0616)$ & $(0.0193)$ & $(0.0308)$ & $(0.0101)$ & $(0.0174)$ \\
& $0.311^{* *}$ & $0.462^{* * *}$ & $0.123^{*}$ & 0.136 & 0.0510 & 0.0246 \\
Saving rate & $(0.125)$ & $(0.170)$ & $(0.0727)$ & $(0.0955)$ & $(0.0368)$ & $(0.0323)$ \\
& $-0.0368^{* * *}$ & $-0.0473^{* * *}$ & $-0.0128^{* * *}$ & $-0.0149^{* * *}$ & $-0.00566^{* *}$ & -0.00322 \\
Tot & $(0.00855)$ & $(0.0118)$ & $(0.00343)$ & $(0.00468)$ & $(0.00267)$ & $(0.00320)$ \\
& $-0.818^{* *}$ & $-1.015^{* * *}$ & $-0.223^{* *}$ & $-0.261^{* *}$ & $-0.0883^{*}$ & -0.0504 \\
Off & $(0.337)$ & $(0.373)$ & $(0.0975)$ & $(0.119)$ & $(0.0512)$ & $(0.0594)$ \\
& $0.157^{* * *}$ & $0.225^{* * *}$ & $0.0519^{* *}$ & $0.0825 * * *$ & 0.0185 & 0.0156 \\
FDI & $(0.0515)$ & $(0.0649)$ & $(0.0217)$ & $(0.0313)$ & $(0.0115)$ & $(0.0164)$ \\
& $0.00293^{* *}$ & $0.00458^{* * *}$ & 0.000711 & 0.00113 & $4.49 \mathrm{e}-05$ & $9.56 \mathrm{e}-05$ \\
PORT & $(0.00120)$ & $(0.00174)$ & $(0.000491)$ & $(0.000731)$ & $(0.000227)$ & $(0.000180)$ \\
& $-0.00344^{* *}$ & $-0.0111^{* * *}$ & -0.00113 & $-0.00352^{*}$ & -0.000980 & $-0.00275^{*}$ \\
Ret. Diff. & $(0.00149)$ & $(0.00296)$ & $(0.000744)$ & $(0.00206)$ & $(0.000667)$ & $(0.00141)$ \\
& 0.00812 & -0.00241 & 0.00937 & 0.00920 & 0.00581 & 0.00394 \\
M/GDP & $(0.0154)$ & $(0.0187)$ & $(0.00618)$ & $(0.00916)$ & $(0.00360)$ & $(0.00415)$ \\
& $0.00357^{*}$ & $0.00518^{* *}$ & $0.00233^{* * *}$ & $0.00234^{*}$ & $0.00118^{* *}$ & 0.000582 \\
& $(0.00196)$ & $(0.00249)$ & $(0.000795)$ & $(0.00127)$ & $(0.000592)$ & $(0.000676)$ \\
\hline N. Obs. & 172 & 118 & 187 & 132 & 199 & 140 \\
Sample & All & Dev & All & Dev & All & Dev \\
Threshold & $4 \%$ & $4 \%$ & $6 \%$ & $6 \%$ & $8 \%$ & $8 \%$ \\
\hline Robust sta & & & & &
\end{tabular}

Robust standard errors in parentheses

$* * * \mathrm{p}<0.01, * * \mathrm{p}<0.05, * \mathrm{p}<0.1$

Table A4: Correlates of current account episodes (Exports)

\begin{tabular}{lcccccc}
\hline & $(1)$ & $(2)$ & $(3)$ & $(4)$ & $(5)$ & $(6)$ \\
\hline Ln(GDP) & $-0.0720^{* *}$ & -0.0316 & -0.0210 & -0.00788 & $-0.0151^{*}$ & -0.00379 \\
Ln(GDPPC) & $(0.0315)$ & $(0.0390)$ & $(0.0130)$ & $(0.0214)$ & $(0.00842)$ & $(0.00790)$ \\
& $0.0821^{* *}$ & $0.133^{* *}$ & 0.0270 & $0.0596^{*}$ & $0.0191^{*}$ & 0.0189 \\
CA Open & $(0.0402)$ & $(0.0616)$ & $(0.0194)$ & $(0.0320)$ & $(0.0109)$ & $(0.0179)$ \\
& $0.342^{* * *}$ & $0.483^{* * *}$ & $0.143^{* *}$ & $0.163^{*}$ & $0.0640^{*}$ & 0.0304 \\
Saving rate & $(0.127)$ & $(0.172)$ & $(0.0713)$ & $(0.0940)$ & $(0.0389)$ & $(0.0358)$ \\
& $-0.0319^{* * *}$ & $-0.0424^{* * *}$ & $-0.0120^{* * *}$ & $-0.0140^{* * *}$ & $-0.00548^{* *}$ & -0.00305 \\
Tot & $(0.00784)$ & $(0.0102)$ & $(0.00336)$ & $(0.00456)$ & $(0.00251)$ & $(0.00294)$ \\
& $-0.782^{* *}$ & $-0.947^{* * *}$ & $-0.208^{* *}$ & $-0.247^{*}$ & -0.0714 & -0.0411 \\
Off & $(0.318)$ & $(0.363)$ & $(0.106)$ & $(0.128)$ & $(0.0511)$ & $(0.0503)$ \\
& $0.168^{* * *}$ & $0.246^{* * *}$ & $0.0639^{* * *}$ & $0.0973^{* * *}$ & $0.0245^{*}$ & 0.0189 \\
FDI & $(0.0515)$ & $(0.0690)$ & $(0.0223)$ & $(0.0331)$ & $(0.0130)$ & $(0.0186)$ \\
& $0.0328^{* * *}$ & $0.00463^{* *}$ & $0.000859^{*}$ & $0.00126^{*}$ & $9.98 \mathrm{e}-05$ & 0.000116 \\
PORT & $(0.00118)$ & $(0.002)$ & $(0.0005)$ & $(0.0008)$ & $(0.0003)$ & $(0.0002)$ \\
& $-0.00362^{* *}$ & $-0.0107^{* * *}$ & -0.00112 & $-0.00361^{*}$ & -0.000935 & $-0.00297 *$ \\
Ret. Diff. & $(0.00149)$ & $(0.00275)$ & $(0.000763)$ & $(0.00204)$ & $(0.000714)$ & $(0.00140)$ \\
& 0.0151 & 0.0160 & $0.0164^{* *}$ & $0.0173^{*}$ & $0.0104^{* *}$ & 0.00626 \\
X/GDP & $(0.0164)$ & $(0.0200)$ & $(0.00721)$ & $(0.0105)$ & $(0.00513)$ & $(0.00611)$ \\
& 0.00114 & 0.00414 & $0.00215^{* * *}$ & 0.00205 & $0.00119^{* *}$ & 0.000553 \\
& $(0.00300)$ & $(0.00267)$ & $(0.000760)$ & $(0.00140)$ & $(0.000560)$ & $(0.000677)$ \\
\hline N. Obs. & 172 & 118 & 187 & 132 & 199 & 140 \\
Sample & All & Dev & All & Dev & All & Dev \\
Threshold & $4 \%$ & $4 \%$ & $6 \%$ & $6 \%$ & $8 \%$ & $8 \%$ \\
\hline R & & & & &
\end{tabular}

Robust standard errors in parentheses

$* * * \mathrm{p}<0.01,{ }^{* *} \mathrm{p}<0.05,{ }^{*} \mathrm{p}<0.1$ 
Table A5: Correlates of current account episodes (developing country dummy)

\begin{tabular}{lccc}
\hline & $(1)$ & $(2)$ & $(3)$ \\
\hline IGDP & $-0.0652^{* *}$ & $-0.0353^{* *}$ & $-0.0240^{* *}$ \\
IGDP_PC & $(0.0294)$ & $(0.0144)$ & $0.0332^{* *}$ \\
& $0.123^{* *}$ & $0.0439^{*}$ & $(0.0158)$ \\
ka_open & $(0.0499)$ & $(0.0240)$ & $0.0707^{*}$ \\
& $0.376^{* * *}$ & $0.153^{* *}$ & $(0.0428)$ \\
save_rate & $(0.122)$ & $(0.0692)$ & $-0.00480^{* *}$ \\
& $-0.0326^{* * *}$ & $-0.00972^{* * *}$ & $(0.00221)$ \\
terms_trade & $(0.00729)$ & $(0.00301)$ & -0.0832 \\
& $-0.747^{* *}$ & $-0.203^{*}$ & $(0.0581)$ \\
Off & $(0.320)$ & $(0.110)$ & $0.0254^{*}$ \\
& $0.181^{* * *}$ & $0.0589^{* *}$ & $(0.0134)$ \\
FDI & $(0.0539)$ & $(0.0229)$ & 0.000303 \\
& $0.00310^{* * *}$ & $0.00120^{* *}$ & $(0.000298)$ \\
PORT & $(0.00118)$ & $(0.000560)$ & $-0.00144^{*}$ \\
& $-0.00394^{* * *}$ & $-0.00169^{* *}$ & $(0.000785)$ \\
DM & $(0.00142)$ & $(0.000740)$ & $0.00900^{*}$ \\
& 0.0164 & $0.0114^{*}$ & $(0.00489)$ \\
Dev & $(0.0152)$ & $(0.00683)$ & 0.0122 \\
& 0.155 & 0.0102 & $(0.0449)$ \\
\hline Observations & $(0.134)$ & $(0.0830)$ & 199 \\
Sample & 172 & 187 & All \\
Threshold & All & $6 \%$ & $8 \%$ \\
\hline
\end{tabular}

Robust standard errors in parentheses

$* * * \mathrm{p}<0.01, * * \mathrm{p}<0.05, * \mathrm{p}<0.1$ 
Table A6: Correlates of current account episodes and regional characteristics

\begin{tabular}{|c|c|c|c|c|}
\hline & $(1)$ & (2) & (3) & (4) \\
\hline \multirow[t]{2}{*}{ lGDP } & $-0.0705^{* *}$ & $-0.0353 * *$ & -0.0167 & $-1.51 \mathrm{e}-08$ \\
\hline & $(0.0307)$ & $(0.0142)$ & $(0.0102)$ & (9. 55e-08) \\
\hline \multirow[t]{2}{*}{ lGDP_PC } & 0. 0995* & 0.0421 & 0.0278 & 2. $31 \mathrm{e}-07$ \\
\hline & $(0.0554)$ & $(0.0281)$ & $(0.0182)$ & (9. 88e-07) \\
\hline \multirow[t]{2}{*}{ ka_open } & $0.381^{* * *}$ & $0.143^{* *}$ & 0.0486 & 1. $17 \mathrm{e}-07$ \\
\hline & $(0.131)$ & (0.0702) & $(0.0367)$ & (5. 52e-07) \\
\hline \multirow[t]{2}{*}{ save_rate } & $-0.0334 * * *$ & $-0.00903^{* * *}$ & $-0.00321 *$ & $-3.79 \mathrm{e}-08$ \\
\hline & $(0.00861)$ & $(0.00304)$ & (0. 00192) & (1. 61e-07) \\
\hline \multirow[t]{2}{*}{ terms_trade } & $-0.657 *$ & $-0.185^{*}$ & -0.0656 & $-3.75 e-07$ \\
\hline & $(0.350)$ & $(0.106)$ & $(0.0430)$ & $(1.61 \mathrm{e}-06)$ \\
\hline \multirow[t]{2}{*}{ Off } & $0.173^{* * *}$ & $0.0489 * *$ & 0.0141 & 5. $40 \mathrm{e}-08$ \\
\hline & $(0.0538)$ & $(0.0221)$ & $(0.00978)$ & (2. 33e-07) \\
\hline \multirow[t]{2}{*}{ FDI } & $0.00277^{*} *$ & $0.000903 *$ & 0.000116 & 2. $01 \mathrm{e}-09$ \\
\hline & $(0.00120)$ & (0. 000527) & (0. 000206) & (8. 12e-09) \\
\hline \multirow[t]{2}{*}{ PORT } & $-0.00339 * *$ & $-0.00146^{* *}$ & $-0.00105 *$ & $-2.47 \mathrm{e}-08$ \\
\hline & $(0.00143)$ & (0. 000733) & (0. 000588) & (9. 39e-08) \\
\hline \multirow[t]{2}{*}{ DM } & 0.00771 & 0.00838 & 0.00523 & 6. 33e-08 \\
\hline & $(0.0162)$ & $(0.00632)$ & (0. 00355) & (2. 69e-07) \\
\hline \multirow[t]{2}{*}{ SSA } & 0.150 & 0.0424 & 0.0800 & $0.724^{* *}$ \\
\hline & (0. 299) & $(0.168)$ & $(0.169)$ & $(0.312)$ \\
\hline \multirow[t]{2}{*}{ ASIA } & 0.0790 & -0.0174 & 0.00972 & \\
\hline & $(0.306)$ & $(0.110)$ & $(0.0827)$ & \\
\hline \multirow[t]{2}{*}{ MNA } & 0.218 & $-0.0517 *$ & -0.0131 & $0.532 *$ \\
\hline & $(0.294)$ & $(0.0310)$ & $(0.0172)$ & $(0.322)$ \\
\hline \multirow[t]{2}{*}{ LAC } & -0.0474 & -0.0358 & -0.0157 & 0.314 \\
\hline & $(0.182)$ & $(0.0670)$ & $(0.0252)$ & $(0.317)$ \\
\hline \multirow[t]{2}{*}{ EME } & $0.444 *$ & 0.151 & 0.151 & $0.902 * * *$ \\
\hline & $(0.257)$ & $(0.198)$ & $(0.181)$ & $(0.140)$ \\
\hline Observations & 172 & 187 & 199 & 177 \\
\hline Sample & All & All & All & All \\
\hline Threshold & $4 \%$ & $6 \%$ & $8 \%$ & $10 \%$ \\
\hline
\end{tabular}

Robust standard errors in parentheses

${ }^{* * *} \mathrm{p}<0.01,{ }^{* *} \mathrm{p}<0.05,{ }^{*} \mathrm{p}<0.1$ 
Table A7: Correlates of current account episodes LIC Dummy

\begin{tabular}{lcccccccc}
\hline & $(1)$ & $(2)$ & $(3)$ & $(4)$ & $(5)$ & $(6)$ & $(7)$ & $(8)$ \\
\hline Ln(GDP) & $-0.0781^{* * *}$ & -0.0585 & $-0.0364^{* * *}$ & -0.0172 & $-0.0245^{* *}$ & -0.00575 & -0.000357 & -0.000220 \\
& $(0.0262)$ & $(0.0365)$ & $(0.0120)$ & $(0.0187)$ & $(0.0103)$ & $(0.00987)$ & $(0.000687)$ & $(0.00115)$ \\
Ln(GDPPC) & $0.0817^{* *}$ & $0.155^{* *}$ & $0.0462^{* *}$ & $0.0899^{* *}$ & $0.0309^{* *}$ & 0.0257 & 0.000678 & 0.00285 \\
& $(0.0394)$ & $(0.0701)$ & $(0.0196)$ & $(0.0357)$ & $(0.0129)$ & $(0.0290)$ & $(0.00117)$ & $(0.00518)$ \\
CA Open & $0.357 * * *$ & $0.532^{* * *}$ & $0.149^{* *}$ & $0.165^{*}$ & 0.0690 & 0.0270 & $7.53 \mathrm{e}-05$ & 0.000457 \\
& $(0.120)$ & $(0.172)$ & $(0.0677)$ & $(0.0882)$ & $(0.0428)$ & $(0.0360)$ & $(0.00110)$ & $(0.00330)$ \\
Saving rate & $-0.0315^{* * *}$ & $-0.0374^{* * *}$ & $-0.00896^{* * *}$ & $-0.0105^{* * *}$ & $-0.00457 * *$ & -0.00229 & -0.000190 & -0.000608 \\
& $(0.00697)$ & $(0.00932)$ & $(0.00306)$ & $(0.00403)$ & $(0.00232)$ & $(0.00261)$ & $(0.000328)$ & $(0.00113)$ \\
Tot & $-0.766^{* *}$ & $-0.944^{* * *}$ & $-0.208^{*}$ & $-0.244 *$ & -0.0836 & -0.0387 & -0.00205 & -0.00544 \\
& $(0.313)$ & $(0.364)$ & $(0.112)$ & $(0.133)$ & $(0.0578)$ & $(0.0514)$ & $(0.00366)$ & $(0.0102)$ \\
Off & $0.163^{* * *}$ & $0.236^{* * *}$ & $0.0564^{* * *}$ & $0.0925^{* * *}$ & $0.0239 *$ & 0.0173 & 0.000223 & 0.000969 \\
& $(0.0507)$ & $(0.0657)$ & $(0.0206)$ & $(0.0316)$ & $(0.0126)$ & $(0.0198)$ & $(0.000385)$ & $(0.00169)$ \\
FDI & $0.00327 * * *$ & $0.00497 * * *$ & $0.00128^{* *}$ & $0.00169 * *$ & 0.000316 & 0.000203 & $1.19 \mathrm{e}-05$ & $3.61 \mathrm{e}-05$ \\
& $(0.00117)$ & $(0.00182)$ & $(0.000553)$ & $(0.000783)$ & $(0.000296)$ & $(0.000252)$ & $(2.06 \mathrm{e}-05)$ & $(6.34 \mathrm{e}-05)$ \\
PORT & $0.00378^{* * *}$ & $-0.0109 * * *$ & $-0.00165 * *$ & $-0.00386^{* *}$ & $-0.00141^{*}$ & $-0.00299 *$ & $-9.77 \mathrm{e}-05$ & -0.000408 \\
& $(0.00142)$ & $(0.00253)$ & $(0.000755)$ & $(0.00190)$ & $(0.000782)$ & $(0.00172)$ & $(0.000128)$ & $(0.000514)$ \\
Ret. Diff. & 0.0132 & 0.00743 & $0.0111^{*}$ & 0.0129 & $0.00870^{*}$ & 0.00494 & 0.000288 & 0.00104 \\
& $(0.0150)$ & $(0.0178)$ & $(0.00668)$ & $(0.00940)$ & $(0.00482)$ & $(0.00555)$ & $(0.000470)$ & $(0.00177)$ \\
LIC & -0.0532 & 0.00694 & 0.0445 & 0.140 & 0.00421 & 0.0271 & $2.85 \mathrm{e}-05$ & 0.00143 \\
& $(0.127)$ & $(0.184)$ & $(0.0963)$ & $(0.152)$ & $(0.0440)$ & $(0.0557)$ & $(0.00120)$ & $(0.00642)$ \\
\hline Observations & 172 & 118 & 187 & 132 & 199 & 140 & 205 & 146 \\
Sample & All & Dev & All & Dev & All & Dev & All & Dev \\
Threshold & $4 \%$ & $4 \%$ & $6 \%$ & $6 \%$ & $8 \%$ & $8 \%$ & $10 \%$ & $10 \%$ \\
\hline
\end{tabular}

Robust standard errors in parentheses

$* * * \mathrm{p}<0.01, * * \mathrm{p}<0.05, * \mathrm{p}<0.1$ 
Table A8: Evolution of growth post $4 \%$ episodes, no controls

\begin{tabular}{|c|c|c|c|c|c|c|c|c|c|c|}
\hline & (1) & (2) & (3) & (4) & (5) & (6) & (7) & (8) & (9) & (10) \\
\hline & GR1 & GR2 & GR3 & GR4 & GR5 & GR6 & GR7 & GR8 & GR9 & GR10 \\
\hline EPI & $0.0205^{* * *}$ & $0.0154^{* * *}$ & $0.0130 * * *$ & $0.00778 * *$ & 0.00395 & $-5.80 \mathrm{e}-05$ & -0.000682 & -0.000866 & -0.000186 & -0.00275 \\
\hline Constant & $\begin{array}{c}0.00742^{* * * *} \\
(0.00265)\end{array}$ & $\begin{array}{c}0.00762 * * * \\
(0.00237)\end{array}$ & $\begin{array}{c}0.00989 * * * \\
(0.00214)\end{array}$ & $\begin{array}{l}0.0130 * * * \\
(0.00205)\end{array}$ & $\begin{array}{l}0.0158 * * * \\
(0.00188)\end{array}$ & $\begin{array}{l}0.0190^{* * * *} \\
(0.00185)\end{array}$ & $\begin{array}{c}0.0200 * * * \\
(0.00179)\end{array}$ & $\begin{array}{c}0.0206 * * * \\
(0.00164)\end{array}$ & $\begin{array}{l}0.0190 * * * \\
(0.00155)\end{array}$ & $\begin{array}{l}0.0194^{* * *} \\
(0.00148)\end{array}$ \\
\hline $\begin{array}{l}\text { Observations } \\
\text { R-sagared }\end{array}$ & 365 & $\begin{array}{l}364 \\
0 \quad 034\end{array}$ & 361 & 361 & 356 & 349 & 343 & 349 & 348 & $\begin{array}{c}334 \\
0 \quad 003\end{array}$ \\
\hline & (11) & (12) & (13) & (14) & (15) & (16) & (17) & (18) & (19) & $(20)$ \\
\hline EPI & $\begin{array}{c}\text { GR11 } \\
-0.00329 \\
(0.00277)\end{array}$ & $\begin{array}{c}\text { GRI2 } \\
-0.00261 \\
(0.00270)\end{array}$ & $\begin{array}{c}\text { GR13 } \\
-0.00141 \\
(0.00271)\end{array}$ & $\begin{array}{c}\text { GR14 } \\
0.000515 \\
(0.00282)\end{array}$ & $\begin{array}{c}\text { GR15 } \\
-0.000874 \\
(0.00283)\end{array}$ & $\begin{array}{c}\text { GR16 } \\
-0.00145 \\
(0.00287)\end{array}$ & $\begin{array}{c}\text { GR17 } \\
-0.00214 \\
(0.00284)\end{array}$ & $\begin{array}{c}\text { GR18 } \\
-0.00149 \\
(0.00269)\end{array}$ & $\begin{array}{c}\text { GR19 } \\
-0.000419 \\
(0.00268)\end{array}$ & $\begin{array}{c}\text { GR20 } \\
-0.000913 \\
(0.00263)\end{array}$ \\
\hline Constant & $\begin{array}{c}0.0185^{* * *} \\
(0.00145)\end{array}$ & $\begin{array}{c}0.0180 * * * \\
(0.00140)\end{array}$ & $\begin{array}{c}0.0173^{* * *} \\
(0.00140)\end{array}$ & $\begin{array}{c}0.0144 * * * \\
(0.00169)\end{array}$ & $\begin{array}{c}0.0152^{* * *} \\
(0.00168)\end{array}$ & $\begin{array}{c}0.0163^{* * *} \\
(0.00168)\end{array}$ & $\begin{array}{c}0.0173^{* * *} \\
(0.00167)\end{array}$ & $\begin{array}{c}0.0174 * * * \\
(0.00155)\end{array}$ & $\begin{array}{c}0.0165^{* * *} \\
(0.00152)\end{array}$ & $\begin{array}{c}0.0168^{* * *} \\
(0.00148)\end{array}$ \\
\hline $\begin{array}{l}\text { Observations } \\
\text { R-squared }\end{array}$ & $\begin{array}{c}327 \\
0.004\end{array}$ & $\begin{array}{c}321 \\
0.003\end{array}$ & $\begin{array}{c}317 \\
0.001\end{array}$ & $\begin{array}{c}230 \\
0.000\end{array}$ & $\begin{array}{c}220 \\
0.000\end{array}$ & $\begin{array}{c}212 \\
0.001\end{array}$ & $\begin{array}{c}209 \\
0.003\end{array}$ & $\begin{array}{c}217 \\
0.001\end{array}$ & $\begin{array}{c}216 \\
0.000\end{array}$ & $\begin{array}{c}213 \\
0.001\end{array}$ \\
\hline
\end{tabular}

Standard errors in parentheses, ${ }^{* * *} \mathrm{p}<0.01,{ }^{* *} \mathrm{p}<0.05,{ }^{*} \mathrm{p}<0.1$

Table A9: Evolution of growth post $6 \%$ episodes, no controls

\begin{tabular}{|c|c|c|c|c|c|c|c|c|c|c|}
\hline & (1) & (2) & (3) & (4) & (5) & (6) & (7) & (8) & (9) & (10) \\
\hline & GR1 & GR2 & GR3 & GR4 & GR5 & GR6 & GR7 & GR8 & GR9 & GR10 \\
\hline EPI & $\begin{array}{c}0.0151^{* * *} \\
(0.00570)\end{array}$ & $\begin{array}{l}0.0127^{* *} \\
(0.00507)\end{array}$ & $\begin{array}{l}0.0115^{* *} \\
(0.00457)\end{array}$ & $\begin{array}{c}0.00569 \\
(0.00434)\end{array}$ & $\begin{array}{c}0.00158 \\
(0.00397)\end{array}$ & $\begin{array}{c}-0.00240 \\
(0.00386)\end{array}$ & $\begin{array}{c}-0.00222 \\
(0.00368)\end{array}$ & $\begin{array}{c}-0.00180 \\
(0.00342)\end{array}$ & $\begin{array}{c}-0.000880 \\
(0.00322)\end{array}$ & $\begin{array}{c}-0.00325 \\
(0.00332)\end{array}$ \\
\hline Constant & $\begin{array}{c}0.00970^{* * *} \\
(0.00243)\end{array}$ & $\begin{array}{c}0.00956^{* * *} \\
(0.00216)\end{array}$ & $\begin{array}{c}0.0113^{* * *} \\
(0.00196)\end{array}$ & $\begin{array}{c}0.0143 * * * \\
(0.00186)\end{array}$ & $\begin{array}{c}0.0169 * * * \\
(0.00171)\end{array}$ & $\begin{array}{l}0.0197 * * * \\
(0.00168)\end{array}$ & $\begin{array}{c}0.0205^{* * *} \\
(0.00162)\end{array}$ & $\begin{array}{c}0.0207 * * * \\
(0.00149)\end{array}$ & $\begin{array}{c}0.0191 * * * \\
(0.00141)\end{array}$ & $\begin{array}{c}0.0193 * * * \\
(0.00136)\end{array}$ \\
\hline $\begin{array}{l}\text { Observations } \\
\text { R-squared }\end{array}$ & $\begin{array}{c}370 \\
0.019\end{array}$ & $\begin{array}{c}369 \\
0.017\end{array}$ & $\begin{array}{c}366 \\
0.017\end{array}$ & $\begin{array}{c}366 \\
0.005\end{array}$ & $\begin{array}{c}360 \\
0.000\end{array}$ & $\begin{array}{c}353 \\
0.001\end{array}$ & $\begin{array}{c}346 \\
0.001\end{array}$ & $\begin{array}{c}353 \\
0.001\end{array}$ & $\begin{array}{c}352 \\
0.000\end{array}$ & $\begin{array}{c}342 \\
0.003\end{array}$ \\
\hline & $\begin{array}{c}\text { (11) } \\
\text { GR11 }\end{array}$ & $\begin{array}{c}(12) \\
\text { GR12 }\end{array}$ & $\begin{array}{c}\text { (13) } \\
\text { GR13 }\end{array}$ & $\begin{array}{c}(14) \\
\text { GR14 }\end{array}$ & $\begin{array}{c}\text { (15) } \\
\text { GR15 }\end{array}$ & $\begin{array}{c}(16) \\
\text { GR16 }\end{array}$ & $\begin{array}{c}(17) \\
\text { GR17 }\end{array}$ & $\begin{array}{c}(18) \\
\text { GR18 }\end{array}$ & $\begin{array}{c}\text { (19) } \\
\text { GR19 }\end{array}$ & $\begin{array}{c}(20) \\
\text { GR20 }\end{array}$ \\
\hline EPI & $\begin{array}{l}-0.00474 \\
(0.00337)\end{array}$ & $\begin{array}{l}-0.00479 \\
(0.00331)\end{array}$ & $\begin{array}{l}-0.00347 \\
(0.00333)\end{array}$ & $\begin{array}{c}-0.000848 \\
(0.00338)\end{array}$ & $\begin{array}{c}-0.00352 \\
(0.00330)\end{array}$ & $\begin{array}{l}-0.00419 \\
(0.00332)\end{array}$ & $\begin{array}{l}-0.00484 \\
(0.00325)\end{array}$ & $\begin{array}{l}-0.00399 \\
(0.00307)\end{array}$ & $\begin{array}{l}-0.00314 \\
(0.00304)\end{array}$ & $\begin{array}{l}-0.00388 \\
(0.00299)\end{array}$ \\
\hline Constant & $\begin{array}{c}0.0184 * * * \\
(0.00133)\end{array}$ & $\begin{array}{c}0.0179 * * * \\
(0.00127)\end{array}$ & $\begin{array}{c}0.0174 * * * \\
(0.00128)\end{array}$ & $\begin{array}{c}0.0146 * * * \\
(0.00154)\end{array}$ & $\begin{array}{c}0.0157 * * * \\
(0.00150)\end{array}$ & $\begin{array}{c}0.0168 * * * \\
(0.00149)\end{array}$ & $\begin{array}{c}0.0179 * * * \\
(0.00147)\end{array}$ & $\begin{array}{c}0.0179 * * * \\
(0.00136)\end{array}$ & $\begin{array}{c}0.0170^{* * * *} \\
(0.00133)\end{array}$ & $\begin{array}{c}0.0173^{* * *} \\
(0.00129)\end{array}$ \\
\hline $\begin{array}{l}\text { Observations } \\
\text { R-squared }\end{array}$ & $\begin{array}{c}336 \\
0.006 \\
\end{array}$ & $\begin{array}{c}331 \\
0.006 \\
\end{array}$ & $\begin{array}{c}326 \\
0.003 \\
\end{array}$ & $\begin{array}{c}226 \\
0.000 \\
\end{array}$ & $\begin{array}{c}219 \\
0.005 \\
\end{array}$ & $\begin{array}{c}213 \\
0.007 \\
\end{array}$ & $\begin{array}{c}210 \\
0.011 \\
\end{array}$ & $\begin{array}{c}218 \\
0.008 \\
\end{array}$ & $\begin{array}{c}219 \\
0.005 \\
\end{array}$ & $\begin{array}{c}217 \\
0.004 \\
\end{array}$ \\
\hline
\end{tabular}


Table A10: Evolution of growth post $8 \%$ episodes, no controls

\begin{tabular}{|c|c|c|c|c|c|c|c|c|c|c|}
\hline & $\begin{array}{c}(1) \\
\text { GR1 } \\
\end{array}$ & $\begin{array}{c}(2) \\
\text { GR2 } \\
\end{array}$ & $\begin{array}{c}\text { (3) } \\
\text { GR3 }\end{array}$ & $\begin{array}{c}\text { (4) } \\
\text { GR4 }\end{array}$ & $\begin{array}{c}(5) \\
\text { GR5 }\end{array}$ & $\begin{array}{c}\text { (6) } \\
\text { GR6 }\end{array}$ & $\begin{array}{c}\text { (7) } \\
\text { GR7 }\end{array}$ & $\begin{array}{c}\text { (8) } \\
\text { GR8 }\end{array}$ & $\begin{array}{c}\text { (9) } \\
\text { GR9 }\end{array}$ & $\begin{array}{c}\text { (10) } \\
\text { GR10 }\end{array}$ \\
\hline \multirow[t]{2}{*}{ EPI } & $0.0142^{* *}$ & $0.0137 * *$ & $0.0133^{* *}$ & 0.00832 & 0.00489 & 0.000799 & 0.000638 & 0.00125 & 0.00225 & 1. 31e- 05 \\
\hline & $(0.00687)$ & $(0.00619)$ & $(0.00562)$ & $(0.00534)$ & $(0.00490)$ & $(0.00474)$ & $(0.00452)$ & $(0.00418)$ & $(0.00395)$ & $(0.00415)$ \\
\hline \multirow[t]{2}{*}{ Constant } & $0.0103^{* * *}$ & $0.00960^{* * *}$ & $0.0110^{* * *}$ & $0.0140^{* * *}$ & $0.0165^{* * *}$ & 0. $0191 * * *$ & 0. $0199 * * *$ & $0.0201^{* * *}$ & $0.0185^{* * *}$ & 0. $0188^{* * *}$ \\
\hline & $(0.00233)$ & $(0.00210)$ & $(0.00192)$ & $(0.00182)$ & $(0.00169)$ & $(0.00165)$ & $(0.00159)$ & $(0.00145)$ & $(0.00137)$ & $(0.00131)$ \\
\hline Observations & 365 & 364 & 361 & 361 & 355 & 348 & 341 & 348 & 347 & 339 \\
\hline \multirow[t]{3}{*}{ R-squared } & 0.012 & 0.013 & 0.015 & 0.007 & 0.003 & 0.000 & 0.000 & 0.000 & 0.001 & 0.000 \\
\hline & (11) & (12) & (13) & (14) & (15) & (16) & (17) & (18) & (19) & (20) \\
\hline & GR11 & GR12 & GR13 & GR14 & GR15 & GR16 & GR17 & GR18 & GR19 & GR20 \\
\hline \multirow[t]{2}{*}{ EPI } & -0.00340 & -0.00359 & -0.00361 & -0.000908 & -0.00479 & -0.00651 & $-0.00694 *$ & -0.00467 & -0.00461 & -0.00635 \\
\hline & (0. 00433) & $(0.00436)$ & $(0.00434)$ & $(0.00429)$ & (0. 00424) & $(0.00415)$ & $(0.00408)$ & $(0.00403)$ & $(0.00403)$ & $(0.00406)$ \\
\hline \multirow[t]{2}{*}{ Constant } & $0.0180^{* * *}$ & $0.0175^{* * *}$ & 0. $0171^{* * *}$ & $0.0142^{* * * *}$ & $0.0153^{* * *}$ & 0. $0166^{* * * *}$ & $0.0176^{* * * *}$ & $0.0175^{* * * *}$ & $0.0166^{* * *}$ & $0.0170^{* * *}$ \\
\hline & $(0.00128)$ & (0. 00123) & (0. 00123) & (0. 00148) & $(0.00142)$ & $(0.00141)$ & $(0.00140)$ & $(0.00130)$ & $(0.00127)$ & $(0.00122)$ \\
\hline Observations & 333 & 328 & 323 & 220 & 213 & 208 & 205 & 212 & 213 & 211 \\
\hline R-squared & 0.002 & 0.002 & 0.002 & 0.000 & 0.006 & 0.012 & 0.014 & 0.006 & 0.006 & 0.012 \\
\hline
\end{tabular}

Standard errors in parentheses, ${ }^{* * *} \mathrm{p}<0.01,{ }^{* *} \mathrm{p}<0.05,{ }^{*} \mathrm{p}<0.1$

Table A11: Evolution of growth post $10 \%$ episodes, no controls

\begin{tabular}{|c|c|c|c|c|c|c|c|c|c|c|}
\hline & (1) & (2) & (3) & (4) & (5) & (6) & (7) & (8) & (9) & (10) \\
\hline & GR1 & GR2 & GR3 & GR4 & GR5 & GR6 & GR7 & GR8 & GR9 & GR10 \\
\hline EPI & $\begin{array}{c}0.00274 \\
(0.00841)\end{array}$ & $\begin{array}{c}0.00724 \\
(0.00759)\end{array}$ & $\begin{array}{c}0.00703 \\
(0.00691)\end{array}$ & $\begin{array}{l}0.000365 \\
(0.00654)\end{array}$ & $\begin{array}{c}-0.00224 \\
(0.00598)\end{array}$ & $\begin{array}{c}-0.00420 \\
(0.00579)\end{array}$ & $\begin{array}{c}-0.00338 \\
(0.00552)\end{array}$ & $\begin{array}{c}-0.00164 \\
(0.00505)\end{array}$ & $\begin{array}{c}0.00117 \\
(0.00476)\end{array}$ & $\begin{array}{c}0.00109 \\
(0.00502)\end{array}$ \\
\hline Constant & $\begin{array}{c}0.0105^{* * *} \\
(0.00230)\end{array}$ & $\begin{array}{c}0.00937^{* * *} \\
(0.00208)\end{array}$ & $\begin{array}{c}0.0108 * * * \\
(0.00190)\end{array}$ & $\begin{array}{c}0.0137 * * * \\
(0.00180)\end{array}$ & $\begin{array}{c}0.0162 * * * \\
(0.00166)\end{array}$ & $\begin{array}{c}0.0187 * * * \\
(0.00162)\end{array}$ & $\begin{array}{c}0.0195^{* * *} \\
(0.00156)\end{array}$ & $\begin{array}{c}0.0199 * * * \\
(0.00142)\end{array}$ & $\begin{array}{c}0.0182^{* * *} \\
(0.00133)\end{array}$ & $\begin{array}{c}0.0184^{* * * *} \\
(0.00128)\end{array}$ \\
\hline $\begin{array}{l}\text { Observations } \\
\text { R-squared }\end{array}$ & $\begin{array}{c}361 \\
0.000\end{array}$ & $\begin{array}{c}360 \\
0.003\end{array}$ & $\begin{array}{c}357 \\
0.003\end{array}$ & $\begin{array}{c}357 \\
0.000\end{array}$ & $\begin{array}{c}351 \\
0.000\end{array}$ & $\begin{array}{c}344 \\
0.002\end{array}$ & $\begin{array}{c}337 \\
0.001\end{array}$ & $\begin{array}{c}344 \\
0.000\end{array}$ & $\begin{array}{c}343 \\
0.000\end{array}$ & $\begin{array}{c}338 \\
0.000\end{array}$ \\
\hline & $\begin{array}{c}\text { (11) } \\
\text { GR11 }\end{array}$ & $\begin{array}{c}\text { (12) } \\
\text { GR12 }\end{array}$ & $\begin{array}{c}\text { (13) } \\
\text { GR13 }\end{array}$ & $\begin{array}{c}(14) \\
\text { GR14 }\end{array}$ & $\begin{array}{c}\text { (15) } \\
\text { GR15 }\end{array}$ & $\begin{array}{c}\text { (16) } \\
\text { GR16 }\end{array}$ & $\begin{array}{c}\text { (17) } \\
\text { GR17 }\end{array}$ & $\begin{array}{c}\text { (18) } \\
\text { GR18 }\end{array}$ & $\begin{array}{c}(19) \\
\text { GR19 }\end{array}$ & $\begin{array}{c}(20) \\
\text { GR20 }\end{array}$ \\
\hline EPI & $\begin{array}{c}-0.00125 \\
(0.00527)\end{array}$ & $\begin{array}{c}-0.00225 \\
(0.00535)\end{array}$ & $\begin{array}{c}-0.00196 \\
(0.00533)\end{array}$ & $\begin{array}{l}0.000292 \\
(0.00506)\end{array}$ & $\begin{array}{l}-0.00294 \\
(0.00496)\end{array}$ & $\begin{array}{c}-0.00502 \\
(0.00485)\end{array}$ & $\begin{array}{l}-0.00589 \\
(0.00473)\end{array}$ & $\begin{array}{l}-0.00272 \\
(0.00473)\end{array}$ & $\begin{array}{l}-0.00612 \\
(0.00492)\end{array}$ & $\begin{array}{c}-0.00961^{*} \\
(0.00512)\end{array}$ \\
\hline Constant & $\begin{array}{c}0.0176^{* * *} \\
(0.00126)\end{array}$ & $\begin{array}{c}0.0171 * * * \\
(0.00122)\end{array}$ & $\begin{array}{c}0.0168 * * * \\
(0.00122)\end{array}$ & $\begin{array}{c}0.0134 * * * \\
(0.00146)\end{array}$ & $\begin{array}{c}0.0146 * * * \\
(0.00141)\end{array}$ & $\begin{array}{c}0.0158 * * * \\
(0.00140)\end{array}$ & $\begin{array}{c}0.0169 * * * \\
(0.00138)\end{array}$ & $\begin{array}{c}0.0168 * * * \\
(0.00127)\end{array}$ & $\begin{array}{c}0.0159 * * * \\
(0.00123)\end{array}$ & $\begin{array}{c}0.0163 * * * \\
(0.00118)\end{array}$ \\
\hline $\begin{array}{l}\text { Observations } \\
\text { R-squared }\end{array}$ & $\begin{array}{c}334 \\
0.000\end{array}$ & $\begin{array}{c}329 \\
0.001\end{array}$ & $\begin{array}{c}324 \\
0.000\end{array}$ & $\begin{array}{c}216 \\
0.000\end{array}$ & $\begin{array}{c}210 \\
0.002 \\
\end{array}$ & $\begin{array}{c}205 \\
0.005\end{array}$ & $\begin{array}{c}201 \\
0.008 \\
\end{array}$ & $\begin{array}{c}209 \\
0.002\end{array}$ & $\begin{array}{c}209 \\
0.007\end{array}$ & $\begin{array}{c}207 \\
0.017\end{array}$ \\
\hline
\end{tabular}


Table A12: Evolution of growth post $4 \%$ episodes, controlling for education and saving rate

\begin{tabular}{|c|c|c|c|c|c|c|c|c|c|c|}
\hline & (1) & (2) & (3) & (4) & (5) & (6) & (7) & (8) & (9) & (10) \\
\hline & GR1 & GR2 & GR3 & GR4 & GR5 & GR6 & GR7 & GR8 & GR9 & GR10 \\
\hline EPI & $\begin{array}{c}0.0284^{* * *} \\
(0.00521)\end{array}$ & $\begin{array}{c}0.0203^{* * *} \\
(0.00485)\end{array}$ & $\begin{array}{c}0.0168^{* * *} \\
(0.00430)\end{array}$ & $\begin{array}{c}0.0152^{* * *} \\
(0.00417)\end{array}$ & $\begin{array}{c}0.0112 * * * \\
(0.00363)\end{array}$ & $\begin{array}{c}0.00743^{* *} \\
(0.00341)\end{array}$ & $\begin{array}{c}0.00531 \\
(0.00331)\end{array}$ & $\begin{array}{c}0.00441 \\
(0.00316)\end{array}$ & 0. 00411 & $\begin{array}{c}0.00129 \\
0.00301)\end{array}$ \\
\hline LY & $\begin{array}{c}-0.00694^{* * *} \\
(0.00220)\end{array}$ & $\begin{array}{c}-0.00805 * * * \\
(0.00204)\end{array}$ & $\begin{array}{c}-0.00870^{* * *} \\
(0.00180)\end{array}$ & $\begin{array}{c}-0.00736 * * * \\
(0.00175)\end{array}$ & $\begin{array}{c}-0.00793 * * * \\
(0.00153)\end{array}$ & $\begin{array}{c}-0.00811^{* * *} \\
(0.00144)\end{array}$ & $\begin{array}{c}-0.00808^{* * *} \\
(0.00140)\end{array}$ & $\begin{array}{c}-0.00812 * * * \\
(0.00134)\end{array}$ & $\begin{array}{c}-0.00733^{* * *} \\
(0.00127)\end{array}$ & $\begin{array}{c}-0.00679 * * * \\
(0.00127)\end{array}$ \\
\hline iyr_sch & $\begin{array}{c}0.00308^{* * *} \\
(0.00114)\end{array}$ & $\begin{array}{c}0.00377^{* * *} \\
(0.00105)\end{array}$ & $\begin{array}{c}0.00408^{* * *} \\
(0.000930)\end{array}$ & $\begin{array}{l}0.00467 * * * \\
(0.000902)\end{array}$ & $\begin{array}{c}0.00493^{* * *} \\
(0.000789)\end{array}$ & $\begin{array}{c}0.00477^{* * *} \\
(0.000741)\end{array}$ & $\begin{array}{c}0.00481^{* * *} \\
(0.000719)\end{array}$ & $\begin{array}{c}0.00454^{* * *} \\
(0.000690)\end{array}$ & $\begin{array}{c}0.00378^{* * *} \\
(0.000656)\end{array}$ & $\begin{array}{r}0.00346 * * * \\
(0.000647)\end{array}$ \\
\hline save_rate & $\begin{array}{c}0.00126^{* * * *} \\
(0.000288)\end{array}$ & $\begin{array}{c}0.000973^{* * *} \\
(0.000268)\end{array}$ & $\begin{array}{c}0.000871^{* * *} \\
(0.000239)\end{array}$ & $\begin{array}{c}0.000938^{* * *} \\
(0.000231)\end{array}$ & $\begin{array}{c}0.000877^{* * *} \\
(0.000202)\end{array}$ & $\begin{array}{c}0.000861 * * * \\
(0.000192)\end{array}$ & $\begin{array}{c}0.000772^{* * *} \\
(0.000187)\end{array}$ & $\begin{array}{c}0.000760^{* * *} \\
(0.000178)\end{array}$ & $\begin{array}{c}0.000698^{* * *} \\
(0.000169)\end{array}$ & $\begin{array}{c}0.000674^{* * * *} \\
(0.000167)\end{array}$ \\
\hline Constant & $\begin{array}{c}0.0229 \\
(0.0142)\end{array}$ & $\begin{array}{c}0.0339 * * \\
(0.0132)\end{array}$ & $\begin{array}{c}0.0412^{* * *} \\
(0.0118)\end{array}$ & $\begin{array}{c}0.0266 * * \\
(0.0114)\end{array}$ & $\begin{array}{c}0.0333^{* * *} \\
(0.00998)\end{array}$ & $\begin{array}{c}0.0390^{* * *} \\
(0.00937)\end{array}$ & $\begin{array}{c}0.0420^{* * *} \\
(0.00913)\end{array}$ & $\begin{array}{c}0.0446^{* * *} \\
(0.00862)\end{array}$ & $\begin{array}{c}0.0426^{* * *} \\
(0.00819)\end{array}$ & $\begin{array}{c}0.0414 * * * \\
(0.00809)\end{array}$ \\
\hline Observations & 227 & 228 & 227 & 227 & 226 & 225 & 223 & 224 & 223 & 214 \\
\hline R-squared & 0.187 & 0.159 & 0.181 & 0.191 & 0.225 & 0.227 & 0.226 & 0.224 & 0.195 & 0.178 \\
\hline & $\begin{array}{l}\text { (11) } \\
\text { GR11 }\end{array}$ & $\begin{array}{l}(12) \\
\text { GR12 }\end{array}$ & $\begin{array}{c}(13) \\
\text { GR13 }\end{array}$ & $\begin{array}{c}(14) \\
\text { GR14 }\end{array}$ & $\begin{array}{c}\text { (15) } \\
\text { GR15 }\end{array}$ & $\begin{array}{c}(16) \\
\text { GR16 }\end{array}$ & $\begin{array}{c}\text { (17) } \\
\text { GR17 }\end{array}$ & $\begin{array}{c}(18) \\
\text { GR18 }\end{array}$ & $\begin{array}{l}(19) \\
\text { GR19 }\end{array}$ & $\begin{array}{c}\text { (20) } \\
\text { GR20 }\end{array}$ \\
\hline$\overline{\text { EPI }}$ & $\begin{array}{l}0.000480 \\
(0.00298)\end{array}$ & $\begin{array}{l}0.000986 \\
(0.00296)\end{array}$ & $\begin{array}{l}0.000789 \\
(0.00297)\end{array}$ & $\begin{array}{c}0.00217 \\
(0.00366)\end{array}$ & $\begin{array}{c}0.00203 \\
(0.00365)\end{array}$ & $\begin{array}{c}0.00129 \\
(0.00368)\end{array}$ & $\begin{array}{c}0.00105 \\
(0.00369)\end{array}$ & $\begin{array}{c}0.00133 \\
(0.00347)\end{array}$ & $\begin{array}{c}0.00323 \\
(0.00357)\end{array}$ & $\begin{array}{c}0.00341 \\
(0.00356)\end{array}$ \\
\hline LY & $\begin{array}{c}-0.00688 * * * \\
(0.00123)\end{array}$ & $\begin{array}{c}-0.00697 * * * \\
(0.00121)\end{array}$ & $\begin{array}{c}-0.00748^{* * *} \\
(0.00121)\end{array}$ & $\begin{array}{c}-0.00364^{* *} \\
(0.00182)\end{array}$ & $\begin{array}{c}-0.00406^{* *} \\
(0.00180)\end{array}$ & $\begin{array}{c}-0.00433^{* *} \\
(0.00182)\end{array}$ & $\begin{array}{c}-0.00417^{* *} \\
(0.00181)\end{array}$ & $\begin{array}{c}-0.00415^{* *} \\
(0.00169)\end{array}$ & $\begin{array}{c}-0.00458^{* * *} \\
(0.00170)\end{array}$ & $\begin{array}{c}-0.00443^{* *} \\
(0.00171)\end{array}$ \\
\hline iyr_sch & $\begin{array}{c}0.00357^{* * * *} \\
(0.000629)\end{array}$ & $\begin{array}{c}0.00338 * * * \\
(0.000624)\end{array}$ & $\begin{array}{c}0.00350^{* * *} * \\
(0.000614)\end{array}$ & $\begin{array}{c}0.00244 * * * \\
(0.000870)\end{array}$ & $\begin{array}{c}0.00254 * * * \\
(0.000891)\end{array}$ & $\begin{array}{c}0.00257 * * * \\
(0.000915)\end{array}$ & $\begin{array}{c}0.00250^{* * *} \\
(0.000905)\end{array}$ & $\begin{array}{c}0.00232 * * * \\
(0.000864)\end{array}$ & $\begin{array}{c}0.00251 * * * \\
(0.000881)\end{array}$ & $\begin{array}{c}0.00242 * * * \\
(0.000883)\end{array}$ \\
\hline save_rate & $\begin{array}{c}0.000594 * * * \\
(0.000166)\end{array}$ & $\begin{array}{c}0.000562^{* * *} \\
(0.000163)\end{array}$ & $\begin{array}{c}0.000518^{* * *} \\
(0.000162)\end{array}$ & $\begin{array}{l}0.000509 * * \\
(0.000209)\end{array}$ & $\begin{array}{l}0.000517 * * \\
(0.000206)\end{array}$ & $\begin{array}{c}0.000557^{* * *} \\
(0.000205)\end{array}$ & $\begin{array}{c}0.000545^{* * *} \\
(0.000202)\end{array}$ & $\begin{array}{c}0.000542 * * * \\
(0.000193)\end{array}$ & $\begin{array}{c}0.000579 * * * \\
(0.000198)\end{array}$ & $\begin{array}{c}0.000569 * * * \\
(0.000199)\end{array}$ \\
\hline Constant & $\begin{array}{c}0.0426^{* * *} \\
(0.00788)\end{array}$ & $\begin{array}{c}0.0445^{* * *} \\
(0.00772)\end{array}$ & $\begin{array}{c}0.0490 * * * \\
(0.00786)\end{array}$ & $\begin{array}{l}0.0227 * \\
(0.0123)\end{array}$ & $\begin{array}{l}0.0261^{* *} \\
(0.0121)\end{array}$ & $\begin{array}{l}0.0285^{* *} \\
(0.0122)\end{array}$ & $\begin{array}{l}0.0285^{* *} \\
(0.0122)\end{array}$ & $\begin{array}{c}0.0296^{* * *} \\
(0.0112)\end{array}$ & $\begin{array}{c}0.0301^{* * *} \\
(0.0111)\end{array}$ & $\begin{array}{c}0.0300^{* * *} \\
(0.0112)\end{array}$ \\
\hline Observations & 212 & 209 & 206 & 143 & 137 & 133 & 131 & 134 & 133 & 131 \\
\hline R-squared & 0.183 & 0.176 & 0.190 & 0.100 & 0.109 & 0.117 & 0.117 & 0.113 & 0.118 & 0.113 \\
\hline
\end{tabular}


Table A13: Evolution of growth post $6 \%$ episodes, controlling for education and saving rate

\begin{tabular}{|c|c|c|c|c|c|c|c|c|c|c|}
\hline & (1) & (2) & (3) & (4) & (5) & (6) & (7) & (8) & (9) & (10) \\
\hline & GR1 & GR2 & GR3 & GR4 & GR5 & GR6 & GR7 & GR8 & GR9 & GR10 \\
\hline EPI & $\begin{array}{c}0.0240 * * * \\
(0.00619)\end{array}$ & $\begin{array}{c}0.0198^{* * *} \\
(0.00561)\end{array}$ & $\begin{array}{l}0.0161 * * * \\
(0.00498)\end{array}$ & $\begin{array}{c}0.0144 * * * \\
(0.00485)\end{array}$ & $\begin{array}{l}0.0102 * * \\
(0.00429)\end{array}$ & $\begin{array}{l}0.00685 * \\
(0.00402)\end{array}$ & $\begin{array}{c}0.00579 \\
(0.00388)\end{array}$ & $\begin{array}{c}0.00518 \\
(0.00367)\end{array}$ & $\begin{array}{c}0.00442 \\
(0.00349)\end{array}$ & $\begin{array}{c}0.00302 \\
(0.00370)\end{array}$ \\
\hline LY & $\begin{array}{c}-0.00728^{* * *} \\
(0.00214)\end{array}$ & $\begin{array}{c}-0.00848^{* * *} \\
(0.00193)\end{array}$ & $\begin{array}{c}-0.00928 * * * \\
(0.00172)\end{array}$ & $\begin{array}{c}-0.00793 * * * \\
(0.00167)\end{array}$ & $\begin{array}{c}-0.00842 * * * \\
(0.00148)\end{array}$ & $\begin{array}{c}-0.00839 * * * \\
(0.00139)\end{array}$ & $\begin{array}{c}-0.00812 * * * \\
(0.00134)\end{array}$ & $\begin{array}{c}-0.00805 * * * \\
(0.00127)\end{array}$ & $\begin{array}{c}-0.00727 * * * \\
(0.00121)\end{array}$ & $\begin{array}{c}-0.00702^{* * *} \\
(0.00122)\end{array}$ \\
\hline iyr_sch & $\begin{array}{c}0.00247^{* *} \\
(0.00111)\end{array}$ & $\begin{array}{c}0.00370^{* * *} \\
(0.000999)\end{array}$ & $\begin{array}{l}0.00424^{* * *} \\
(0.000884)\end{array}$ & $\begin{array}{l}0.00477 * * * \\
(0.000860)\end{array}$ & $\begin{array}{l}0.00495 * * * \\
(0.000764)\end{array}$ & $\begin{array}{c}0.00476 * * * \\
(0.000717)\end{array}$ & $\begin{array}{l}0.00474^{* * *} \\
(0.000690)\end{array}$ & $\begin{array}{l}0.00448^{* * *} \\
(0.000656)\end{array}$ & $\begin{array}{l}0.00366 * * * \\
(0.000624)\end{array}$ & $\begin{array}{c}0.00345^{* * *} \\
(0.000622)\end{array}$ \\
\hline save_rate & $\begin{array}{c}0.00115 * * * \\
(0.000278)\end{array}$ & $\begin{array}{c}0.000859 * * * \\
(0.000252)\end{array}$ & $\begin{array}{c}0.000709 * * * \\
(0.000225)\end{array}$ & $\begin{array}{c}0.000801^{* * *} \\
(0.000219)\end{array}$ & $\begin{array}{c}0.000762^{* * *} \\
(0.000193)\end{array}$ & $\begin{array}{c}0.000759 * * * \\
(0.000184)\end{array}$ & $\begin{array}{c}0.000682^{* * *} \\
(0.000178)\end{array}$ & $\begin{array}{c}0.000689 * * * \\
(0.000168)\end{array}$ & $\begin{array}{c}0.000653^{* * * *} \\
(0.000160)\end{array}$ & $\begin{array}{c}0.000687^{* * *} \\
(0.000159)\end{array}$ \\
\hline Constant & $\begin{array}{c}0.0356^{* * *} \\
(0.0133)\end{array}$ & $\begin{array}{c}0.0431^{* * *} \\
(0.0121)\end{array}$ & $\begin{array}{c}0.0509 * * * \\
(0.0108)\end{array}$ & $\begin{array}{c}0.0359 * * * \\
(0.0105)\end{array}$ & $\begin{array}{c}0.0414^{* * *} \\
(0.00932)\end{array}$ & $\begin{array}{c}0.0449 * * * \\
(0.00875)\end{array}$ & $\begin{array}{c}0.0454^{* * *} \\
(0.00848)\end{array}$ & $\begin{array}{c}0.0463 * * * \\
(0.00792)\end{array}$ & $\begin{array}{c}0.0441^{* * *} \\
(0.00754)\end{array}$ & $\begin{array}{c}0.0432^{* * *} \\
(0.00747)\end{array}$ \\
\hline $\begin{array}{l}\text { Observations } \\
\text { R-squared }\end{array}$ & $\begin{array}{c}234 \\
0.133 \\
\end{array}$ & $\begin{array}{c}235 \\
0.136 \\
\end{array}$ & $\begin{array}{c}234 \\
0.161 \\
\end{array}$ & $\begin{array}{c}234 \\
0.171 \\
\end{array}$ & $\begin{array}{c}233 \\
0.203 \\
\end{array}$ & $\begin{array}{c}232 \\
0.211 \\
\end{array}$ & $\begin{array}{c}229 \\
0.215 \\
\end{array}$ & $\begin{array}{c}231 \\
0.218 \\
\end{array}$ & $\begin{array}{c}230 \\
0.188 \\
\end{array}$ & $\begin{array}{c}223 \\
0.184 \\
\end{array}$ \\
\hline & $\begin{array}{c}(11) \\
\text { GR11 } \\
\end{array}$ & $\begin{array}{c}(12) \\
\text { GR12 } \\
\end{array}$ & $\begin{array}{c}(13) \\
\text { GR13 } \\
\end{array}$ & $\begin{array}{c}(14) \\
\text { GR14 }\end{array}$ & $\begin{array}{c}(15) \\
\text { GR15 }\end{array}$ & $\begin{array}{c}(16) \\
\text { GR16 }\end{array}$ & $\begin{array}{c}(17) \\
\text { GR17 }\end{array}$ & $\begin{array}{c}(18) \\
\text { GR18 } \\
\end{array}$ & $\begin{array}{c}\text { (19) } \\
\text { GR19 }\end{array}$ & $\begin{array}{c}(20) \\
\text { GR20 }\end{array}$ \\
\hline EPI & $\begin{array}{c}0.00172 \\
(0.00365)\end{array}$ & $\begin{array}{c}0.00212 \\
(0.00369)\end{array}$ & $\begin{array}{c}0.00245 \\
(0.00373)\end{array}$ & $\begin{array}{c}0.00489 \\
(0.00417)\end{array}$ & $\begin{array}{c}0.00294 \\
(0.00405)\end{array}$ & $\begin{array}{c}0.00202 \\
(0.00414)\end{array}$ & $\begin{array}{c}0.00118 \\
(0.00404)\end{array}$ & $\begin{array}{c}0.00176 \\
(0.00381)\end{array}$ & $\begin{array}{c}0.00285 \\
(0.00392)\end{array}$ & $\begin{array}{c}0.00371 \\
(0.00391)\end{array}$ \\
\hline LY & $\begin{array}{c}-0.00695^{* * *} \\
(0.00118)\end{array}$ & $\begin{array}{c}-0.00700^{* * *} \\
(0.00116)\end{array}$ & $\begin{array}{c}-0.00749 * * * \\
(0.00115)\end{array}$ & $\begin{array}{c}-0.00360^{* *} \\
(0.00167)\end{array}$ & $\begin{array}{c}-0.00392 * * \\
(0.00161)\end{array}$ & $\begin{array}{c}-0.00414^{* *} \\
(0.00163)\end{array}$ & $\begin{array}{c}-0.00387 * * \\
(0.00160)\end{array}$ & $\begin{array}{c}-0.00383^{* *} \\
(0.00148)\end{array}$ & $\begin{array}{c}-0.00414^{* * * *} \\
(0.00149)\end{array}$ & $\begin{array}{c}-0.00425^{* * * *} \\
(0.00147)\end{array}$ \\
\hline iyr_sch & $\begin{array}{l}0.00342 * * * \\
(0.000602)\end{array}$ & $\begin{array}{c}0.00323 * * * \\
(0.000596)\end{array}$ & $\begin{array}{c}0.00336 * * * \\
(0.000588)\end{array}$ & $\begin{array}{c}0.00258^{* * *} \\
(0.000829)\end{array}$ & $\begin{array}{c}0.00245 * * * \\
(0.000807)\end{array}$ & $\begin{array}{l}0.00246 * * * \\
(0.000831)\end{array}$ & $\begin{array}{c}0.00234^{* * *} \\
(0.000810)\end{array}$ & $\begin{array}{c}0.00221^{* * *} \\
(0.000766)\end{array}$ & $\begin{array}{c}0.00224^{* * *} \\
(0.000764)\end{array}$ & $\begin{array}{l}0.00222^{* * *} \\
(0.000756)\end{array}$ \\
\hline save_rate & $\begin{array}{c}0.000618^{* * * *} \\
(0.000157)\end{array}$ & $\begin{array}{c}0.000581^{* * *} \\
(0.000154)\end{array}$ & $\begin{array}{c}0.000552 * * * \\
(0.000153)\end{array}$ & $\begin{array}{c}0.000556 * * * \\
(0.000201)\end{array}$ & $\begin{array}{c}0.000560 * * * \\
(0.000191)\end{array}$ & $\begin{array}{c}\text { 0. } 000585^{* * *} \\
(0.000189)\end{array}$ & $\begin{array}{c}0.000555 * * * \\
(0.000184)\end{array}$ & $\begin{array}{c}0.000549 * * * \\
(0.000174)\end{array}$ & $\begin{array}{c}0.000553 * * * \\
(0.000178)\end{array}$ & $\begin{array}{c}0.000566^{* * *} \\
(0.000177)\end{array}$ \\
\hline Constant & $\begin{array}{c}0.0436 * * * \\
(0.00721)\end{array}$ & $\begin{array}{c}0.0454 * * * \\
(0.00704)\end{array}$ & $\begin{array}{c}0.0493 * * * \\
(0.00713)\end{array}$ & $\begin{array}{c}0.0206 * \\
(0.0106)\end{array}$ & $\begin{array}{c}0.0249^{* *} \\
(0.0102)\end{array}$ & $\begin{array}{c}0.0272^{* * *} \\
(0.0102)\end{array}$ & $\begin{array}{c}0.0269 * * * \\
(0.0100)\end{array}$ & $\begin{array}{c}0.0275 * * * \\
(0.00921)\end{array}$ & $\begin{array}{c}0.0287 * * * \\
(0.00915)\end{array}$ & $\begin{array}{c}0.0299 * * * \\
(0.00905)\end{array}$ \\
\hline $\begin{array}{l}\text { Observations } \\
\text { R-squared }\end{array}$ & $\begin{array}{c}222 \\
0.184\end{array}$ & $\begin{array}{c}219 \\
0.178\end{array}$ & $\begin{array}{c}215 \\
0.194\end{array}$ & $\begin{array}{c}141 \\
0.121\end{array}$ & $\begin{array}{c}139 \\
0.122\end{array}$ & $\begin{array}{c}136 \\
0.129\end{array}$ & $\begin{array}{c}135 \\
0.127\end{array}$ & $\begin{array}{c}138 \\
0.125\end{array}$ & $\begin{array}{c}138 \\
0.120\end{array}$ & $\begin{array}{c}137 \\
0.122\end{array}$ \\
\hline
\end{tabular}


Table A14: Evolution of growth post $8 \%$ episodes, controlling for education and saving rate

\begin{tabular}{|c|c|c|c|c|c|c|c|c|c|c|}
\hline & (1) & (2) & (3) & (4) & (5) & (6) & (7) & (8) & (9) & (10) \\
\hline & GR1 & GR2 & GR3 & GR4 & GR5 & GR6 & GR7 & GR8 & GR9 & GR10 \\
\hline LY & $\begin{array}{c}-0.00774^{* * *} \\
(0.00215)\end{array}$ & $\begin{array}{c}-0.00878^{* * *} \\
(0.00195)\end{array}$ & $\begin{array}{c}-0.00945 * * * \\
(0.00174)\end{array}$ & $\begin{array}{c}-0.00828 * * * \\
(0.00168)\end{array}$ & $\begin{array}{c}-0.00878^{* * *} \\
(0.00148)\end{array}$ & $\begin{array}{c}-0.00872^{* * *} \\
(0.00138)\end{array}$ & $\begin{array}{c}-0.00838^{* * *} \\
(0.00133)\end{array}$ & $\begin{array}{c}-0.00822 * * * \\
(0.00126)\end{array}$ & $\begin{array}{c}-0.00738^{* * * *} \\
(0.00120)\end{array}$ & $\begin{array}{c}-0.00718^{* * *} \\
(0.00120)\end{array}$ \\
\hline iyr_sch & $\begin{array}{c}0.00262^{* *} \\
(0.00113)\end{array}$ & $\begin{array}{c}0.00377^{* * *} \\
(0.00102)\end{array}$ & $\begin{array}{c}0.00439 * * * \\
(0.000907)\end{array}$ & $\begin{array}{c}0.00491 * * * \\
(0.000872)\end{array}$ & $\begin{array}{c}0.00506^{* * *} \\
(0.000768)\end{array}$ & $\begin{array}{c}0.00487 * * * \\
(0.000717)\end{array}$ & $\begin{array}{c}0.00483^{* * *} \\
(0.000690)\end{array}$ & $\begin{array}{c}0.00453^{* * *} \\
(0.000656)\end{array}$ & $\begin{array}{c}0.00367^{* * *} \\
(0.000625)\end{array}$ & $\begin{array}{c}0.00349^{* * *} \\
(0.000622)\end{array}$ \\
\hline save_rate & $\begin{array}{l}0.00107 * * * \\
(0.000278)\end{array}$ & $\begin{array}{c}0.000833^{* * * *} \\
(0.000252)\end{array}$ & $\begin{array}{c}0.000721^{* * *} \\
(0.000227)\end{array}$ & $\begin{array}{c}0.000802^{* * *} \\
(0.000218)\end{array}$ & $\begin{array}{c}0.000756^{* * *} \\
(0.000191)\end{array}$ & $\begin{array}{c}0.000754^{* * *} \\
(0.000181)\end{array}$ & $\begin{array}{c}0.000677^{* * *} \\
(0.000175)\end{array}$ & $\begin{array}{c}0.000683^{* * *} \\
(0.000165)\end{array}$ & $\begin{array}{c}0.000635 * * * \\
(0.000157)\end{array}$ & $\begin{array}{c}0.000664 * * * \\
(0.000157)\end{array}$ \\
\hline Constant & $\begin{array}{c}0.0411 * * * \\
(0.0132)\end{array}$ & $\begin{array}{c}0.0460 * * * \\
(0.0120)\end{array}$ & $\begin{array}{c}0.0509^{* * *} \\
(0.0108)\end{array}$ & $\begin{array}{c}0.0381^{* * *} \\
(0.0104)\end{array}$ & $\begin{array}{c}0.0441^{* * *} \\
(0.00914)\end{array}$ & $\begin{array}{c}0.0471^{* * *} \\
(0.00853)\end{array}$ & $\begin{array}{c}0.0473^{* * *} \\
(0.00826)\end{array}$ & $\begin{array}{c}0.0477 * * * \\
(0.00773)\end{array}$ & $\begin{array}{c}0.0456^{* * *} \\
(0.00736)\end{array}$ & $\begin{array}{c}0.0449 * * * \\
(0.00723)\end{array}$ \\
\hline Observations & 233 & 234 & 233 & 233 & 232 & 231 & 228 & 230 & 229 & 224 \\
\hline R-squared & 0.109 & 0.121 & 0.154 & 0.172 & 0.207 & 0.219 & 0.222 & 0.223 & 0.189 & 0.184 \\
\hline & $\begin{array}{l}(11) \\
\text { GR11 }\end{array}$ & $\begin{array}{l}(12) \\
\text { GR12 }\end{array}$ & $\begin{array}{l}\text { (13) } \\
\text { GR13 }\end{array}$ & $\begin{array}{l}(14) \\
\text { GR14 }\end{array}$ & $\begin{array}{c}\text { (15) } \\
\text { GR15 }\end{array}$ & $\begin{array}{c}(16) \\
\text { GR16 }\end{array}$ & $\begin{array}{l}(17) \\
\text { GR17 }\end{array}$ & $\begin{array}{c}(18) \\
\text { GR18 }\end{array}$ & $\begin{array}{c}(19) \\
\text { GR19 }\end{array}$ & $\begin{array}{c}(20) \\
\text { GR20 }\end{array}$ \\
\hline$\overline{\mathrm{EPI}}$ & $\begin{array}{c}0.00184 \\
(0.00455)\end{array}$ & $\begin{array}{c}0.00251 \\
(0.00475)\end{array}$ & $\begin{array}{c}0.00150 \\
(0.00467)\end{array}$ & $\begin{array}{c}0.00559 \\
(0.00522)\end{array}$ & $\begin{array}{c}0.00252 \\
(0.00519)\end{array}$ & $\begin{array}{l}0.000544 \\
(0.00516)\end{array}$ & $\begin{array}{l}-0.000185 \\
(0.00503)\end{array}$ & $\begin{array}{l}0.000455 \\
(0.00476)\end{array}$ & $\begin{array}{l}0.000867 \\
(0.00502)\end{array}$ & $\begin{array}{c}0.00193 \\
(0.00507)\end{array}$ \\
\hline LY & $\begin{array}{c}-0.00713^{* * *} \\
(0.00116)\end{array}$ & $\begin{array}{c}-0.00712^{* * * *} \\
(0.00115)\end{array}$ & $\begin{array}{c}-0.00772^{* * *} \\
(0.00113)\end{array}$ & $\begin{array}{c}-0.00433^{* *} \\
(0.00166)\end{array}$ & $\begin{array}{c}-0.00460^{* * *} \\
(0.00161)\end{array}$ & $\begin{array}{c}-0.00463^{* * * *} \\
(0.00162)\end{array}$ & $\begin{array}{c}-0.00434^{* * *} \\
(0.00159)\end{array}$ & $\begin{array}{c}-0.00425^{* * *} \\
(0.00148)\end{array}$ & $\begin{array}{c}-0.00457^{* * * *} \\
(0.00149)\end{array}$ & $\begin{array}{c}-0.00468^{* * *} \\
(0.00147)\end{array}$ \\
\hline iyr_sch & $\begin{array}{l}0.00346 * * * \\
(0.000603)\end{array}$ & $\begin{array}{l}0.00327 * * * \\
(0.000601)\end{array}$ & $\begin{array}{c}0.00339 * * * \\
(0.000589)\end{array}$ & $\begin{array}{c}0.00291^{* * *} \\
(0.000855)\end{array}$ & $\begin{array}{l}0.00278^{* * * *} \\
(0.000841)\end{array}$ & $\begin{array}{l}0.00266 * * * \\
(0.000844)\end{array}$ & $\begin{array}{c}0.00252^{* * *} \\
(0.000823)\end{array}$ & $\begin{array}{c}0.00236 * * * \\
(0.000780)\end{array}$ & $\begin{array}{l}0.00241^{* * *} \\
(0.000780)\end{array}$ & $\begin{array}{c}0.00238 * * * \\
(0.000770)\end{array}$ \\
\hline save_rate & $\begin{array}{c}0.000594^{* * *} \\
(0.000156)\end{array}$ & $\begin{array}{c}0.000558^{* * * *} \\
(0.000152)\end{array}$ & $\begin{array}{c}0.000520^{* * *} \\
(0.000151)\end{array}$ & $\begin{array}{l}0.000520^{* *} \\
(0.000199)\end{array}$ & $\begin{array}{c}0.000505^{* * *} \\
(0.000190)\end{array}$ & $\begin{array}{c}0.000516^{* * *} \\
(0.000187)\end{array}$ & $\begin{array}{c}0.000487^{* * *} \\
(0.000183)\end{array}$ & $\begin{array}{c}0.000481^{* * *} \\
(0.000173)\end{array}$ & $\begin{array}{c}0.000472^{* * *} \\
(0.000178)\end{array}$ & $\begin{array}{c}0.000490^{* * *} \\
(0.000176)\end{array}$ \\
\hline Constant & $\begin{array}{l}0.0455^{* * *} \\
(0.00701)\end{array}$ & $\begin{array}{l}0.0468 * * * \\
(0.00685)\end{array}$ & $\begin{array}{l}0.0519 * * * \\
(0.00688)\end{array}$ & $\begin{array}{c}0.0260 * * \\
(0.0103)\end{array}$ & $\begin{array}{l}0.0302^{* * *} \\
(0.00990)\end{array}$ & $\begin{array}{l}0.0320 * * * \\
(0.00988)\end{array}$ & $\begin{array}{l}0.0316 * * * \\
(0.00972)\end{array}$ & $\begin{array}{l}0.0318 * * * \\
(0.00896)\end{array}$ & $\begin{array}{l}0.0334^{* * *} \\
(0.00888)\end{array}$ & $\begin{array}{c}0.0346 * * * \\
(0.00877)\end{array}$ \\
\hline Observations & 223 & 220 & 216 & 139 & 137 & 135 & 134 & 137 & 137 & 136 \\
\hline R-squared & 0.185 & 0.178 & 0.201 & 0.121 & 0.122 & 0.128 & 0.125 & 0.122 & 0.118 & 0.118 \\
\hline
\end{tabular}


Table A15: Evolution of growth post 10\% episodes, controlling for education and saving rate

\begin{tabular}{|c|c|c|c|c|c|c|c|c|c|c|}
\hline & $\begin{array}{c}\text { (1) } \\
\text { GR1 }\end{array}$ & $\begin{array}{c}\text { (2) } \\
\text { GR2 }\end{array}$ & $\begin{array}{l}\text { (3) } \\
\text { GR3 }\end{array}$ & $\begin{array}{c}\text { (4) } \\
\text { GR4 }\end{array}$ & $\begin{array}{l}\text { (5) } \\
\text { GR5 }\end{array}$ & $\begin{array}{c}\text { (6) } \\
\text { GR6 }\end{array}$ & $\begin{array}{c}\text { (7) } \\
\text { GR7 }\end{array}$ & $\begin{array}{c}\text { (8) } \\
\text { GR8 }\end{array}$ & $\begin{array}{c}\text { (9) } \\
\text { GR9 }\end{array}$ & $\begin{array}{c}(10) \\
\text { GR10 }\end{array}$ \\
\hline EPI & 0.000376 & 0.00352 & 0.000108 & -0.00133 & -0.00617 & -0.00614 & -0.00644 & -0.00553 & -0.00492 & -0.00644 \\
\hline & (0. 0101) & $(0.00926)$ & $(0.00830)$ & $(0.00795)$ & $(0.00689)$ & $(0.00651)$ & $(0.00624)$ & (0. 00583) & $(0.00551)$ & $(0.00608)$ \\
\hline LY & $\begin{array}{c}-0.00914^{* * * *} \\
(0.00213)\end{array}$ & $\begin{array}{c}-0.00975^{* * *} \\
(0.00195)\end{array}$ & $\begin{array}{c}-0.0105 * * * \\
(0.00175)\end{array}$ & $\begin{array}{c}-0.00920^{* * * *} \\
(0.00168)\end{array}$ & $\begin{array}{c}-0.00983^{* * * *} \\
(0.00146)\end{array}$ & $\begin{array}{c}-0.00959 * * * \\
(0.00138)\end{array}$ & $\begin{array}{c}-0.00910^{* * * *} \\
(0.00133)\end{array}$ & $\begin{array}{c}-0.00885^{* * * *} \\
(0.00124)\end{array}$ & $\begin{array}{c}-0.00786 * * * \\
(0.00117)\end{array}$ & $\begin{array}{c}-0.00763 * * * \\
(0.00115)\end{array}$ \\
\hline iyr_sch & $\begin{array}{c}0.00341^{* * *} \\
(0.00113)\end{array}$ & $\begin{array}{c}0.00427^{* * *} \\
(0.00103)\end{array}$ & $\begin{array}{c}0.00506^{* * *} \\
(0.000922)\end{array}$ & $\begin{array}{c}0.00548^{* * *} \\
(0.000883)\end{array}$ & $\begin{array}{l}0.00565 * * * \\
(0.000769)\end{array}$ & $\begin{array}{c}0.00536^{* * *} \\
(0.000727)\end{array}$ & $\begin{array}{c}0.00525 * * * \\
(0.000696)\end{array}$ & $\begin{array}{c}0.00492^{* * *} \\
(0.000653)\end{array}$ & $\begin{array}{c}0.00397 * * * \\
(0.000618)\end{array}$ & $\begin{array}{c}0.00388^{* * *} \\
(0.000600)\end{array}$ \\
\hline save_rate & $\begin{array}{c}0.000936 * * * \\
(0.000280)\end{array}$ & $\begin{array}{c}0.000871^{* * * *} \\
(0.000258)\end{array}$ & $\begin{array}{c}0.000645^{* * * *} \\
(0.000232)\end{array}$ & $\begin{array}{c}0.000699 * * * \\
(0.000222)\end{array}$ & $\begin{array}{c}0.000636 * * * \\
(0.000193)\end{array}$ & $\begin{array}{c}0.000657 * * * \\
(0.000185)\end{array}$ & $\begin{array}{c}0.000548^{* * * *} \\
(0.000178)\end{array}$ & $\begin{array}{c}0.000547^{* * *} \\
(0.000166)\end{array}$ & $\begin{array}{c}0.000511^{* * * *} \\
(0.000157)\end{array}$ & $\begin{array}{c}0.000531^{* * *} \\
(0.000156)\end{array}$ \\
\hline Constant & $\begin{array}{c}0.0510^{* * *} \\
(0.0131)\end{array}$ & $\begin{array}{c}0.0503^{* * *} \\
(0.0120)\end{array}$ & $\begin{array}{c}0.0568^{* * *} \\
(0.0109)\end{array}$ & $\begin{array}{c}0.0445^{* * *} \\
(0.0104)\end{array}$ & $\begin{array}{c}0.0518^{* * *} \\
(0.00905)\end{array}$ & $\begin{array}{c}0.0535^{* * *} \\
(0.00856)\end{array}$ & $\begin{array}{c}0.0535 * * * \\
(0.00825)\end{array}$ & $\begin{array}{c}0.0536^{* * *} \\
(0.00761)\end{array}$ & $\begin{array}{l}0.0503 * * * \\
(0.00721)\end{array}$ & $\begin{array}{c}0.0490^{* * *} \\
(0.00700)\end{array}$ \\
\hline Observations & 228 & 229 & 228 & 228 & 227 & 226 & 223 & 225 & 224 & 221 \\
\hline R-squared & 0.099 & 0.124 & 0.159 & 0.182 & 0.237 & 0.246 & 0.247 & 0.249 & 0.210 & 0.218 \\
\hline & $\begin{array}{c}(11) \\
\text { GR11 }\end{array}$ & $\begin{array}{l}(12) \\
\text { GR12 }\end{array}$ & $\begin{array}{c}\text { (13) } \\
\text { GR13 }\end{array}$ & $\begin{array}{c}\text { (14) } \\
\text { GR14 }\end{array}$ & $\begin{array}{c}\text { (15) } \\
\text { GR15 }\end{array}$ & $\begin{array}{c}\text { (16) } \\
\text { GR16 }\end{array}$ & $\begin{array}{c}\text { (17) } \\
\text { GR17 }\end{array}$ & $\begin{array}{c}(18) \\
\text { GR18 }\end{array}$ & $\begin{array}{c}\text { (19) } \\
\text { GR19 }\end{array}$ & $\begin{array}{c}(20) \\
\text { GR20 }\end{array}$ \\
\hline EPI & $\begin{array}{c}-0.00697 \\
(0.00586)\end{array}$ & $\begin{array}{c}-0.00876 \\
(0.00613)\end{array}$ & $\begin{array}{l}-0.00998^{*} \\
(0.00603)\end{array}$ & $\begin{array}{l}-0.00606 \\
(0.00654)\end{array}$ & $\begin{array}{l}-0.00707 \\
(0.00619)\end{array}$ & $\begin{array}{c}-0.00953 \\
(0.00608)\end{array}$ & $\begin{array}{l}-0.0105^{*} \\
(0.00590)\end{array}$ & $\begin{array}{l}-0.00905 \\
(0.00558)\end{array}$ & $\begin{array}{l}-0.0106^{*} \\
(0.00608)\end{array}$ & $\begin{array}{l}-0.00929 * \\
(0.00634)\end{array}$ \\
\hline LY & $\begin{array}{c}-0.00776^{* * *} \\
(0.00111)\end{array}$ & $\begin{array}{c}-0.00777 * * * \\
(0.00109)\end{array}$ & $\begin{array}{c}-0.00834^{* * * *} \\
(0.00107)\end{array}$ & $\begin{array}{c}-0.00488 * * * \\
(0.00164)\end{array}$ & $\begin{array}{c}-0.00555^{* * *} \\
(0.00157)\end{array}$ & $\begin{array}{c}-0.00559 * * * \\
(0.00156)\end{array}$ & $\begin{array}{c}-0.00532 * * * \\
(0.00152)\end{array}$ & $\begin{array}{c}-0.00514 * * * \\
(0.00142)\end{array}$ & $\begin{array}{c}-0.00522^{* * *} \\
(0.00142)\end{array}$ & $\begin{array}{c}-0.00532 * * * \\
(0.00140)\end{array}$ \\
\hline iyr_sch & $\begin{array}{c}0.00389^{* * *} \\
(0.000580)\end{array}$ & $\begin{array}{c}0.00357^{* * *} \\
(0.000576)\end{array}$ & $\begin{array}{c}0.00366^{* * *} \\
(0.000565)\end{array}$ & $\begin{array}{c}0.00308^{* * *} \\
(0.000855)\end{array}$ & $\begin{array}{c}0.00326^{* * *} \\
(0.000817)\end{array}$ & $\begin{array}{c}0.00315^{* * *} \\
(0.000813)\end{array}$ & $\begin{array}{c}0.00303^{* * *} \\
(0.000789)\end{array}$ & $\begin{array}{c}0.00283^{* * *} \\
(0.000746)\end{array}$ & $\begin{array}{c}0.00279^{* * *} \\
(0.000741)\end{array}$ & $\begin{array}{c}0.00275^{* * *} \\
(0.000733)\end{array}$ \\
\hline save_rate & $\begin{array}{c}0.000504^{* * *} \\
(0.000151)\end{array}$ & $\begin{array}{c}0.000484^{* * *} \\
(0.000148)\end{array}$ & $\begin{array}{c}0.000447 * * * \\
(0.000146)\end{array}$ & $\begin{array}{l}0.000385^{*} \\
(0.000197)\end{array}$ & $\begin{array}{l}0.000364^{*} \\
(0.000187)\end{array}$ & $\begin{array}{l}0.000365^{* *} \\
(0.000183)\end{array}$ & $\begin{array}{l}0.000334^{*} \\
(0.000178)\end{array}$ & $\begin{array}{c}0.000336^{* *} \\
(0.000168)\end{array}$ & $\begin{array}{c}0.000286 \\
(0.000174)\end{array}$ & $\begin{array}{l}0.000319 * \\
(0.000173)\end{array}$ \\
\hline Constant & $\begin{array}{c}0.0499 * * * \\
(0.00675)\end{array}$ & $\begin{array}{c}0.0518^{* * *} \\
(0.00659)\end{array}$ & $\begin{array}{c}0.0568^{* * *} \\
(0.00663)\end{array}$ & $\begin{array}{c}0.0323^{* * *} \\
(0.0102)\end{array}$ & $\begin{array}{c}0.0381^{* * *} \\
(0.00976)\end{array}$ & $\begin{array}{c}0.0401^{* * *} \\
(0.00965)\end{array}$ & $\begin{array}{c}0.0399 * * * \\
(0.00944)\end{array}$ & $\begin{array}{c}0.0394^{* * *} \\
(0.00869)\end{array}$ & $\begin{array}{c}0.0404^{* * *} \\
(0.00856)\end{array}$ & $\begin{array}{l}0.0412^{* * *} \\
(0.00847)\end{array}$ \\
\hline Observations & 221 & 219 & 215 & 134 & 133 & 131 & 130 & 133 & 133 & 132 \\
\hline R-squared & 0.228 & 0.227 & 0.252 & 0.137 & 0.158 & 0.172 & 0.175 & 0.168 & 0.168 & 0.160 \\
\hline
\end{tabular}


Table A16: Evolution of growth post 4\% episodes, controlling for education and saving rate, interaction with official flows dummy

\begin{tabular}{|c|c|c|c|c|c|c|c|c|c|c|}
\hline & $\begin{array}{c}1) \\
\text { GR1 }\end{array}$ & $\begin{array}{c}\text { (2) } \\
\text { GR2 }\end{array}$ & $\begin{array}{c}\text { (3) } \\
\text { GR3 }\end{array}$ & $\begin{array}{c}(4) \\
\text { GR4 }\end{array}$ & $\begin{array}{c}\text { (5) } \\
\text { GR5 }\end{array}$ & $\begin{array}{c}\text { (6) } \\
\text { GR6 }\end{array}$ & $\begin{array}{c}\text { (7) } \\
\text { GR7 }\end{array}$ & $\begin{array}{c}\text { (8) } \\
\text { GR8 }\end{array}$ & $\begin{array}{c}\text { (9) } \\
\text { GR9 }\end{array}$ & $\begin{array}{c}(10) \\
\text { GR10 }\end{array}$ \\
\hline EPI*NOFF & $0.0195^{* * *}$ & 0.00986 & 0.00627 & 0.000475 & $-5.38 \mathrm{e}-05$ & -0.00246 & -0.00278 & -0.00111 & -0.000142 & -0.00280 \\
\hline & $(0.00749)$ & $(0.00683)$ & $(0.00625)$ & $(0.00565)$ & $(0.00511)$ & $(0.00474)$ & $(0.00451)$ & $(0.00423)$ & $(0.00402)$ & $(0.00393)$ \\
\hline EPI*OFF & $\begin{array}{l}0.0232 * \\
(0.0124)\end{array}$ & $\begin{array}{c}0.0138 \\
(0.0114)\end{array}$ & $\begin{array}{c}0.0153 \\
(0.0104)\end{array}$ & $\begin{array}{c}0.00953 \\
(0.00938)\end{array}$ & $\begin{array}{c}0.00737 \\
(0.00849)\end{array}$ & $\begin{array}{c}0.00424 \\
(0.00787)\end{array}$ & $\begin{array}{c}0.00253 \\
(0.00749)\end{array}$ & $\begin{array}{c}0.00384 \\
(0.00702)\end{array}$ & $\begin{array}{c}0.00164 \\
(0.00668)\end{array}$ & $\begin{array}{c}-0.00303 \\
(0.00662)\end{array}$ \\
\hline OFF & $\begin{array}{l}-0.0116 \\
(0.0121)\end{array}$ & $\begin{array}{c}-0.0105 \\
(0.0110)\end{array}$ & $\begin{array}{l}-0.0143 \\
(0.0101)\end{array}$ & $\begin{array}{l}-0.0154^{*} \\
(0.00909)\end{array}$ & $\begin{array}{l}-0.0148^{*} \\
(0.00823)\end{array}$ & $\begin{array}{c}-0.0153 * * \\
(0.00763)\end{array}$ & $\begin{array}{c}-0.0158^{* *} \\
(0.00726)\end{array}$ & $\begin{array}{c}-0.0175^{* *} \\
(0.00681)\end{array}$ & $\begin{array}{l}-0.0143^{* *} \\
(0.00648)\end{array}$ & $\begin{array}{c}-0.0123^{* *} \\
(0.00620)\end{array}$ \\
\hline LY & $\begin{array}{c}-0.00774 * * * \\
(0.00285)\end{array}$ & $\begin{array}{c}-0.00904 * * * \\
(0.00260)\end{array}$ & $\begin{array}{c}-0.00835 * * * \\
(0.00238)\end{array}$ & $\begin{array}{c}-0.00848^{* * * *} \\
(0.00215)\end{array}$ & $\begin{array}{c}-0.00829 * * * \\
(0.00195)\end{array}$ & $\begin{array}{c}-0.00900 * * * * \\
(0.00180)\end{array}$ & $\begin{array}{c}-0.00930 * * * \\
(0.00172)\end{array}$ & $\begin{array}{c}-0.00925^{* * *} \\
(0.00161)\end{array}$ & $\begin{array}{c}-0.00854 * * * \\
(0.00153)\end{array}$ & $\begin{array}{c}-0.00767 * * * \\
(0.00151)\end{array}$ \\
\hline iyr_sch & $\begin{array}{c}0.00390^{* * *} \\
(0.00138)\end{array}$ & $\begin{array}{c}0.00450 * * * \\
(0.00126)\end{array}$ & $\begin{array}{c}0.00400^{* * *} \\
(0.00116)\end{array}$ & $\begin{array}{c}0.00447^{* * *} \\
(0.00104)\end{array}$ & $\begin{array}{l}0.00451^{* * *} \\
(0.000945)\end{array}$ & $\begin{array}{l}0.00453^{* * *} \\
(0.000875)\end{array}$ & $\begin{array}{c}0.00463^{* * *} \\
(0.000833)\end{array}$ & $\begin{array}{c}0.00430 * * * \\
(0.000781)\end{array}$ & $\begin{array}{c}0.00370^{* * *} \\
(0.000743)\end{array}$ & $\begin{array}{c}0.00336 * * * \\
(0.000736)\end{array}$ \\
\hline Constant & $\begin{array}{c}0.0538^{* * *} \\
(0.0206)\end{array}$ & $\begin{array}{c}0.0604^{* * *} \\
(0.0188)\end{array}$ & $\begin{array}{c}0.0606 * * * \\
(0.0172)\end{array}$ & $\begin{array}{c}0.0629 * * * \\
(0.0155)\end{array}$ & $\begin{array}{c}0.0620^{* * *} \\
(0.0140)\end{array}$ & $\begin{array}{c}0.0706^{* * *} \\
(0.0130)\end{array}$ & $\begin{array}{c}0.0740^{* * *} \\
(0.0124)\end{array}$ & $\begin{array}{c}0.0753^{* * *} \\
(0.0116)\end{array}$ & $\begin{array}{c}0.0706^{* * *} \\
(0.0110)\end{array}$ & $\begin{array}{c}0.0658 * * * \\
(0.0109)\end{array}$ \\
\hline Observations & 207 & 207 & 207 & 207 & 207 & 207 & 207 & 207 & 207 & 196 \\
\hline R-squared & 0.109 & 0.098 & 0.092 & 0.106 & 0.126 & 0.149 & 0.172 & 0.184 & 0.163 & 0.159 \\
\hline & $\begin{array}{l}\text { (11) } \\
\text { GR11 }\end{array}$ & $\begin{array}{l}\text { (12) } \\
\text { GR12 }\end{array}$ & $\begin{array}{l}\text { (13) } \\
\text { GR13 }\end{array}$ & $\begin{array}{l}\text { (14) } \\
\text { GR14 }\end{array}$ & $\begin{array}{c}\text { (15) } \\
\text { GR15 }\end{array}$ & $\begin{array}{c}\text { (16) } \\
\text { GR16 }\end{array}$ & $\begin{array}{c}\text { (17) } \\
\text { GR17 }\end{array}$ & $\begin{array}{l}\text { (18) } \\
\text { GR18 }\end{array}$ & $\begin{array}{c}\text { (19) } \\
\text { GR19 }\end{array}$ & $\begin{array}{c}(20) \\
\text { GR20 }\end{array}$ \\
\hline EPI*NOFF & $\begin{array}{c}-0.00204 \\
(0.00396)\end{array}$ & $\begin{array}{c}-0.00123 \\
(0.00379)\end{array}$ & $\begin{array}{l}-0.000295 \\
(0.00381)\end{array}$ & $\begin{array}{c}-0.00188 \\
(0.00451)\end{array}$ & $\begin{array}{l}-0.00241 \\
(0.00468)\end{array}$ & $\begin{array}{c}-0.00254 \\
(0.00476)\end{array}$ & $\begin{array}{c}-0.00275 \\
(0.00475)\end{array}$ & $\begin{array}{l}-0.00213 \\
(0.00456)\end{array}$ & $\begin{array}{l}0.000193 \\
(0.00467)\end{array}$ & $\begin{array}{l}0.000617 \\
(0.00459)\end{array}$ \\
\hline EPI*OFF & $\begin{array}{c}-0.00551 \\
(0.00649)\end{array}$ & $\begin{array}{l}-0.00505 \\
(0.00608)\end{array}$ & $\begin{array}{c}-0.00573 \\
(0.00601)\end{array}$ & $\begin{array}{c}-0.00369 \\
(0.00671)\end{array}$ & $\begin{array}{l}-0.00332 \\
(0.00659)\end{array}$ & $\begin{array}{c}-0.00391 \\
(0.00645)\end{array}$ & $\begin{array}{l}-0.00425 \\
(0.00627)\end{array}$ & $\begin{array}{l}-0.00558 \\
(0.00628)\end{array}$ & $\begin{array}{l}-0.00516 \\
(0.00630)\end{array}$ & $\begin{array}{l}-0.00492 \\
(0.00627)\end{array}$ \\
\hline OFF & $\begin{array}{l}-0.0103^{*} \\
(0.00609)\end{array}$ & $\begin{array}{l}-0.00978^{*} \\
(0.00568)\end{array}$ & $\begin{array}{l}-0.00878 \\
(0.00561)\end{array}$ & $\begin{array}{c}-0.0111 \\
(0.00673)\end{array}$ & $\begin{array}{l}-0.0112 * \\
(0.00661)\end{array}$ & $\begin{array}{c}-0.0133^{* *} \\
(0.00646)\end{array}$ & $\begin{array}{c}-0.0132 * * \\
(0.00635)\end{array}$ & $\begin{array}{l}-0.0116^{*} \\
(0.00621)\end{array}$ & $\begin{array}{l}-0.0108^{*} \\
(0.00620)\end{array}$ & $\begin{array}{l}-0.0109 * \\
(0.00610)\end{array}$ \\
\hline LY & $\begin{array}{c}-0.00838^{* * *} \\
(0.00150)\end{array}$ & $\begin{array}{c}-0.00812^{* * *} \\
(0.00141)\end{array}$ & $\begin{array}{c}-0.00835 * * * \\
(0.00139)\end{array}$ & $\begin{array}{c}-0.00647 * * * \\
(0.00198)\end{array}$ & $\begin{array}{c}-0.00681^{* * *} \\
(0.00196)\end{array}$ & $\begin{array}{c}-0.00752^{* * *} \\
(0.00193)\end{array}$ & $\begin{array}{c}-0.00755^{* * *} \\
(0.00192)\end{array}$ & $\begin{array}{c}-0.00745^{* * *} \\
(0.00180)\end{array}$ & $\begin{array}{c}-0.00766^{* * *} \\
(0.00182)\end{array}$ & $\begin{array}{c}-0.00738^{* * *} \\
(0.00181)\end{array}$ \\
\hline iyr_sch & $\begin{array}{c}0.00374 * * * \\
(0.000725)\end{array}$ & $\begin{array}{c}0.00325^{* * *} \\
(0.000692)\end{array}$ & $\begin{array}{c}0.00329 * * * \\
(0.000683)\end{array}$ & $\begin{array}{l}0.00277^{* * *} \\
(0.000892)\end{array}$ & $\begin{array}{c}0.00294^{* * *} \\
(0.000913)\end{array}$ & $\begin{array}{l}0.00294 * * * \\
(0.000920)\end{array}$ & $\begin{array}{c}0.00293^{* * *} \\
(0.000895)\end{array}$ & $\begin{array}{c}0.00268 * * * \\
(0.000865)\end{array}$ & $\begin{array}{c}0.00278 * * * \\
(0.000888)\end{array}$ & $\begin{array}{c}0.00258 * * * \\
(0.000880)\end{array}$ \\
\hline Constant & $\begin{array}{c}0.0681^{* * *} \\
(0.0108)\end{array}$ & $\begin{array}{c}0.0689 * * * \\
(0.0101)\end{array}$ & $\begin{array}{c}0.0703^{* * *} \\
(0.0100)\end{array}$ & $\begin{array}{c}0.0591^{* * *} \\
(0.0147)\end{array}$ & $\begin{array}{c}0.0613^{* * *} \\
(0.0145)\end{array}$ & $\begin{array}{c}0.0689 * * * \\
(0.0142)\end{array}$ & $\begin{array}{c}0.0700^{* * *} \\
(0.0143)\end{array}$ & $\begin{array}{c}0.0707^{* * *} \\
(0.0133)\end{array}$ & $\begin{array}{c}0.0707^{* * *} \\
(0.0133)\end{array}$ & $\begin{array}{c}0.0701^{* * *} \\
(0.0132)\end{array}$ \\
\hline Observations & 194 & 189 & 188 & 135 & 129 & 125 & 123 & 123 & 121 & 119 \\
\hline R-squared & 0.183 & 0.176 & 0.186 & 0.122 & 0.130 & 0.156 & 0.162 & 0.161 & 0.162 & 0.158 \\
\hline
\end{tabular}

Standard errors in parentheses, $* * * \mathrm{p}<0.01, * * \mathrm{p}<0.05, * \mathrm{p}<0.1$ 
Table A17: Evolution of growth post 6\% episodes, controlling for education and saving rate, interaction with official flows dummy

\begin{tabular}{|c|c|c|c|c|c|c|c|c|c|c|}
\hline & $\begin{array}{l}\text { (1) } \\
\text { GR1 }\end{array}$ & $\begin{array}{c}(2) \\
\text { GR2 }\end{array}$ & $\begin{array}{c}\text { (3) } \\
\text { GR3 }\end{array}$ & $\begin{array}{c}\text { (4) } \\
\text { GR4 }\end{array}$ & $\begin{array}{c}\text { (5) } \\
\text { GR5 }\end{array}$ & $\begin{array}{c}6) \\
\text { GR6 }\end{array}$ & $\begin{array}{c}\text { (7) } \\
\text { GR7 }\end{array}$ & $\begin{array}{c}8) \\
\text { GR8 }\end{array}$ & $\begin{array}{c}\text { (9) } \\
\text { GR9 }\end{array}$ & $\begin{array}{l}\text { (10) } \\
\text { GR10 }\end{array}$ \\
\hline EPI*NOFF & $\begin{array}{l}0.0265 * * \\
(0.0104)\end{array}$ & $\begin{array}{l}0.0219 * * \\
(0.00905)\end{array}$ & $\begin{array}{c}0.0184^{* *} \\
(0.00824)\end{array}$ & $\begin{array}{c}0.00947 \\
(0.00775)\end{array}$ & $\begin{array}{c}0.00853 \\
(0.00676)\end{array}$ & $\begin{array}{c}0.00679 \\
(0.00620)\end{array}$ & $\begin{array}{c}0.00735 \\
(0.00578)\end{array}$ & $\begin{array}{c}0.00785 \\
(0.00536)\end{array}$ & $\begin{array}{c}0.00762 \\
(0.00513)\end{array}$ & $\begin{array}{c}0.00384 \\
(0.00516)\end{array}$ \\
\hline EPI*OFF & $\begin{array}{l}0.00901 \\
(0.0107)\end{array}$ & $\begin{array}{l}-0.00312 \\
(0.00934)\end{array}$ & $\begin{array}{c}0.00225 \\
(0.00850)\end{array}$ & $\begin{array}{c}0.00447 \\
(0.00800)\end{array}$ & $\begin{array}{l}-0.00132 \\
(0.00698)\end{array}$ & $\begin{array}{c}-0.00372 \\
(0.00640)\end{array}$ & $\begin{array}{l}-0.00400 \\
(0.00597)\end{array}$ & $\begin{array}{c}-0.00212 \\
(0.00554)\end{array}$ & $\begin{array}{l}-0.00231 \\
(0.00529)\end{array}$ & $\begin{array}{l}-0.00461 \\
(0.00592)\end{array}$ \\
\hline OFF & $\begin{array}{c}-0.00364 \\
(0.00873)\end{array}$ & $\begin{array}{l}0.000716 \\
(0.00762)\end{array}$ & $\begin{array}{l}-0.00589 \\
(0.00694)\end{array}$ & $\begin{array}{l}-0.0124^{*} \\
(0.00653)\end{array}$ & $\begin{array}{c}-0.00964^{*} \\
(0.00569)\end{array}$ & $\begin{array}{c}-0.00992^{*} \\
(0.00523)\end{array}$ & $\begin{array}{c}-0.0112^{* *} \\
(0.00487)\end{array}$ & $\begin{array}{c}-0.0126^{* * * *} \\
(0.00452)\end{array}$ & $\begin{array}{c}-0.0116^{* * *} \\
(0.00432)\end{array}$ & $\begin{array}{c}-0.0124^{* * * *} \\
(0.00426)\end{array}$ \\
\hline LY & $\begin{array}{c}-0.00738 * * * \\
(0.00280)\end{array}$ & $\begin{array}{c}-0.00921^{* * *} \\
(0.00245)\end{array}$ & $\begin{array}{c}-0.00879 * * * \\
(0.00223)\end{array}$ & $\begin{array}{c}-0.00823^{* * *} \\
(0.00210)\end{array}$ & $\begin{array}{c}-0.00870^{* * *} \\
(0.00183)\end{array}$ & $\begin{array}{c}-0.00924^{* * * *} \\
(0.00168)\end{array}$ & $\begin{array}{c}-0.00940^{* * *} \\
(0.00156)\end{array}$ & $\begin{array}{c}-0.00921^{* * * *} \\
(0.00145)\end{array}$ & $\begin{array}{c}-0.00859 * * * \\
(0.00139)\end{array}$ & $\begin{array}{c}-0.00810 * * * \\
(0.00140)\end{array}$ \\
\hline iyr_sch & $\begin{array}{c}0.00338^{* *} \\
(0.00133)\end{array}$ & $\begin{array}{c}0.00444 * * * \\
(0.00116)\end{array}$ & $\begin{array}{c}0.00406^{* * *} \\
(0.00106)\end{array}$ & $\begin{array}{l}0.00435^{* * *} \\
(0.000998)\end{array}$ & $\begin{array}{c}0.00464 * * * \\
(0.000870)\end{array}$ & $\begin{array}{l}0.00466^{* * *} \\
(0.000798)\end{array}$ & $\begin{array}{l}0.00468 * * * \\
(0.000744)\end{array}$ & $\begin{array}{l}0.00440^{* * *} \\
(0.000690)\end{array}$ & $\begin{array}{c}0.00378^{* * *} \\
(0.000660)\end{array}$ & $\begin{array}{c}0.00350 * * * \\
(0.000667)\end{array}$ \\
\hline Constant & $\begin{array}{c}0.0518^{* *} \\
(0.0202)\end{array}$ & $\begin{array}{c}0.0606 * * * \\
(0.0177)\end{array}$ & $\begin{array}{c}0.0627^{* * *} \\
(0.0161)\end{array}$ & $\begin{array}{c}0.0599 * * * \\
(0.0151)\end{array}$ & $\begin{array}{c}0.0634^{* * *} \\
(0.0132)\end{array}$ & $\begin{array}{c}0.0703^{* * *} \\
(0.0121)\end{array}$ & $\begin{array}{c}0.0731^{* * *} \\
(0.0113)\end{array}$ & $\begin{array}{c}0.0730 * * * \\
(0.0105)\end{array}$ & $\begin{array}{c}0.0695 * * * \\
(0.0100)\end{array}$ & $\begin{array}{c}0.0677^{* * *} \\
(0.0101)\end{array}$ \\
\hline $\begin{array}{l}\text { Observations } \\
\text { R-squared }\end{array}$ & $\begin{array}{c}230 \\
0.069\end{array}$ & $\begin{array}{c}230 \\
0.100\end{array}$ & $\begin{array}{c}230 \\
0.100\end{array}$ & $\begin{array}{c}230 \\
0.112\end{array}$ & $\begin{array}{c}230 \\
0.148\end{array}$ & $\begin{array}{c}230 \\
0.174\end{array}$ & $\begin{array}{c}230 \\
0.205\end{array}$ & $\begin{array}{c}230 \\
0.219\end{array}$ & $\begin{array}{c}230 \\
0.196\end{array}$ & $\begin{array}{c}222 \\
0.188\end{array}$ \\
\hline & $\begin{array}{c}(11) \\
\text { GR11 } \\
\end{array}$ & $\begin{array}{c}(12) \\
\text { GR12 } \\
\end{array}$ & $\begin{array}{c}\text { (13) } \\
\text { GR13 }\end{array}$ & $\begin{array}{c}\text { (14) } \\
\text { GR14 }\end{array}$ & $\begin{array}{l}\text { (15) } \\
\text { GR15 }\end{array}$ & $\begin{array}{c}\text { (16) } \\
\text { GR16 }\end{array}$ & $\begin{array}{l}\text { (17) } \\
\text { GR17 }\end{array}$ & $\begin{array}{l}\text { (18) } \\
\text { GR18 }\end{array}$ & $\begin{array}{l}\text { (19) } \\
\text { GR19 }\end{array}$ & $\begin{array}{l}(20) \\
\text { GR20 }\end{array}$ \\
\hline EPI*NOFF & $\begin{array}{c}0.00323 \\
(0.00505)\end{array}$ & $\begin{array}{c}0.00477 \\
(0.00501)\end{array}$ & $\begin{array}{c}0.00752 \\
(0.00515)\end{array}$ & $\begin{array}{c}0.00733 \\
(0.00602)\end{array}$ & $\begin{array}{c}0.00466 \\
(0.00601)\end{array}$ & $\begin{array}{c}0.00650 \\
(0.00618)\end{array}$ & $\begin{array}{c}0.00593 \\
(0.00600)\end{array}$ & $\begin{array}{c}0.00664 \\
(0.00563)\end{array}$ & $\begin{array}{c}0.00858 \\
(0.00558)\end{array}$ & $\begin{array}{c}0.00827 \\
(0.00553)\end{array}$ \\
\hline EPI*OFF & $\begin{array}{l}-0.00811 \\
(0.00593)\end{array}$ & $\begin{array}{l}-0.00845 \\
(0.00549)\end{array}$ & $\begin{array}{l}-0.00816 \\
(0.00541)\end{array}$ & $\begin{array}{l}-0.00267 \\
(0.00573)\end{array}$ & $\begin{array}{l}-0.00475 \\
(0.00548)\end{array}$ & $\begin{array}{c}-0.00601 \\
(0.00530)\end{array}$ & $\begin{array}{c}-0.00704 \\
(0.00517)\end{array}$ & $\begin{array}{l}-0.00725 \\
(0.00486)\end{array}$ & $\begin{array}{c}-0.00802 \\
(0.00495)\end{array}$ & $\begin{array}{l}-0.00639 \\
(0.00505)\end{array}$ \\
\hline OFF & $\begin{array}{c}-0.0114 * * * \\
(0.00416)\end{array}$ & $\begin{array}{c}-0.0108 * * * \\
(0.00387)\end{array}$ & $\begin{array}{c}-0.0107 * * * \\
(0.00382)\end{array}$ & $\begin{array}{c}-0.0151 * * * \\
(0.00482)\end{array}$ & $\begin{array}{c}-0.0142 * * * \\
(0.00459)\end{array}$ & $\begin{array}{c}-0.0155 * * * \\
(0.00445)\end{array}$ & $\begin{array}{c}-0.0149 * * * \\
(0.00442)\end{array}$ & $\begin{array}{c}-0.0141 * * * \\
(0.00407)\end{array}$ & $\begin{array}{c}-0.0133 * * * \\
(0.00404)\end{array}$ & $\begin{array}{c}-0.0139 * * * \\
(0.00400)\end{array}$ \\
\hline LY & $\begin{array}{c}-0.00861 * * * \\
(0.00137)\end{array}$ & $\begin{array}{c}-0.00849 * * * \\
(0.00128)\end{array}$ & $\begin{array}{c}-0.00870^{* * *} \\
(0.00126)\end{array}$ & $\begin{array}{c}-0.00707^{* * *} \\
(0.00180)\end{array}$ & $\begin{array}{c}-0.00752^{* * * *} \\
(0.00173)\end{array}$ & $\begin{array}{c}-0.00833^{* * *} \\
(0.00170)\end{array}$ & $\begin{array}{c}-0.00834^{* * *} \\
(0.00169)\end{array}$ & $\begin{array}{c}-0.00823^{* * *} \\
(0.00156)\end{array}$ & $\begin{array}{c}-0.00827^{* * *} \\
(0.00155)\end{array}$ & $\begin{array}{c}-0.00819 * * * \\
(0.00153)\end{array}$ \\
\hline iyr_sch & $\begin{array}{c}0.00375^{* * *} \\
(0.000651)\end{array}$ & $\begin{array}{c}0.00340 * * * \\
(0.000617)\end{array}$ & $\begin{array}{c}0.00342 * * * \\
(0.000609)\end{array}$ & $\begin{array}{c}0.00297 * * * \\
(0.000831)\end{array}$ & $\begin{array}{c}0.00298 * * * \\
(0.000804)\end{array}$ & $\begin{array}{c}0.00314 * * * \\
(0.000802)\end{array}$ & $\begin{array}{c}0.00313 * * * \\
(0.000781)\end{array}$ & $\begin{array}{c}0.00298 * * * \\
(0.000734)\end{array}$ & $\begin{array}{c}0.00289 * * * \\
(0.000731)\end{array}$ & $\begin{array}{c}0.00277 * * * \\
(0.000725)\end{array}$ \\
\hline Constant & $\begin{array}{c}0.0692 * * * \\
(0.00986)\end{array}$ & $\begin{array}{c}0.0704 * * * \\
(0.00916)\end{array}$ & $\begin{array}{c}0.0718 * * * \\
(0.00905)\end{array}$ & $\begin{array}{c}0.0627 * * * \\
(0.0133)\end{array}$ & $\begin{array}{c}0.0672 * * * \\
(0.0128)\end{array}$ & $\begin{array}{c}0.0746 * * * \\
(0.0124)\end{array}$ & $\begin{array}{c}0.0754 * * * \\
(0.0124)\end{array}$ & $\begin{array}{c}0.0753 * * * \\
(0.0113)\end{array}$ & $\begin{array}{c}0.0749 * * * \\
(0.0112)\end{array}$ & $\begin{array}{c}0.0756 * * * \\
(0.0111)\end{array}$ \\
\hline $\begin{array}{l}\text { Observations } \\
\text { R-squared }\end{array}$ & $\begin{array}{c}221 \\
0209\end{array}$ & $\begin{array}{c}217 \\
0215\end{array}$ & $\begin{array}{c}216 \\
0227\end{array}$ & $\begin{array}{c}146 \\
0195\end{array}$ & 143 & $\begin{array}{c}140 \\
0231\end{array}$ & $\begin{array}{c}137 \\
0237\end{array}$ & $\begin{array}{c}139 \\
0247\end{array}$ & $\begin{array}{c}139 \\
0247\end{array}$ & $\begin{array}{c}138 \\
0240\end{array}$ \\
\hline
\end{tabular}

Standard errors in parentheses, $* * * \mathrm{p}<0.01, * * \mathrm{p}<0.05, * \mathrm{p}<0.1$ 
Table A18: Evolution of growth post $8 \%$ episodes, controlling for education and saving rate, interaction with official flows dummy

\begin{tabular}{|c|c|c|c|c|c|c|c|c|c|c|}
\hline & $\begin{array}{c}1) \\
\text { GR1 }\end{array}$ & $\begin{array}{c}\text { (2) } \\
\text { GR2 }\end{array}$ & $\begin{array}{c}\text { (3) } \\
\text { GR3 }\end{array}$ & $\begin{array}{l}\text { (4) } \\
\text { GR4 }\end{array}$ & $\begin{array}{c}\text { (5) } \\
\text { GR5 }\end{array}$ & $\begin{array}{c}\text { (6) } \\
\text { GR6 }\end{array}$ & $\begin{array}{c}\text { (7) } \\
\text { GR7 }\end{array}$ & $\begin{array}{c}8) \\
\text { GR8 }\end{array}$ & $\begin{array}{c}\text { (9) } \\
\text { GR9 }\end{array}$ & $\begin{array}{c}(10) \\
\text { GR10 }\end{array}$ \\
\hline EPI*NOFF & $0.0253^{*}$ & $0.0219 *$ & 0.0166 & 0.0104 & 0.0107 & 0.00949 & 0.00918 & 0.00974 & 0.00690 & $0.0253^{*}$ \\
\hline & $(0.0136)$ & $(0.0118)$ & (0.0109) & $(0.0101)$ & $(0.00892)$ & $(0.00824)$ & $(0.00774)$ & $(0.00716)$ & $(0.00693)$ & $(0.0136)$ \\
\hline EPI*OFF & 0.00429 & 0.00110 & 0.00909 & 0.00700 & 0.00250 & 0.000425 & 0.000409 & 0.00166 & 0.00154 & 0.00429 \\
\hline & $(0.0112)$ & $(0.00981)$ & $(0.00901)$ & $(0.00836)$ & (0.00739) & $(0.00683)$ & $(0.00641)$ & $(0.00594)$ & $(0.00574)$ & $(0.0112)$ \\
\hline OFF & $\begin{array}{c}-0.00103 \\
(0.00740)\end{array}$ & $\begin{array}{c}-0.00454 \\
(0.00646)\end{array}$ & $\begin{array}{l}-0.0111^{*} \\
(0.00593)\end{array}$ & $\begin{array}{c}-0.0133 * * \\
(0.00551)\end{array}$ & $\begin{array}{c}-0.0121^{* *} \\
(0.00487)\end{array}$ & $\begin{array}{c}-0.0124 * * * \\
(0.00449)\end{array}$ & $\begin{array}{c}-0.0138 * * * \\
(0.00422)\end{array}$ & $\begin{array}{c}-0.0143^{* * * *} \\
(0.00391)\end{array}$ & $\begin{array}{c}-0.0133^{* * * *} \\
(0.00378)\end{array}$ & $\begin{array}{l}-0.00103 \\
(0.00740)\end{array}$ \\
\hline LY & $\begin{array}{c}-0.00630^{* *} \\
(0.00262)\end{array}$ & $\begin{array}{c}-0.00867 * * * \\
(0.00229)\end{array}$ & $\begin{array}{c}-0.00833 * * * \\
(0.00210)\end{array}$ & $\begin{array}{c}-0.00759 * * * \\
(0.00195)\end{array}$ & $\begin{array}{c}-0.00823 * * * \\
(0.00172)\end{array}$ & $\begin{array}{c}-0.00873 * * * \\
(0.00159)\end{array}$ & $\begin{array}{c}-0.00921^{* * * *} \\
(0.00149)\end{array}$ & $\begin{array}{c}-0.00895^{* * *} \\
(0.00138)\end{array}$ & $\begin{array}{c}-0.00832 * * * \\
(0.00134)\end{array}$ & $\begin{array}{c}-0.00630^{* *} \\
(0.00262)\end{array}$ \\
\hline iyr_sch & $\begin{array}{c}0.00252^{* *} \\
(0.00125)\end{array}$ & $\begin{array}{c}0.00379 * * * \\
(0.00109)\end{array}$ & $\begin{array}{c}0.00380^{* * *} \\
(0.00100)\end{array}$ & $\begin{array}{l}0.00404^{* * *} \\
(0.000932)\end{array}$ & $\begin{array}{l}0.00437 * * * \\
(0.000824)\end{array}$ & $\begin{array}{l}0.00446 * * * \\
(0.000761)\end{array}$ & $\begin{array}{c}0.00462^{* * *} \\
(0.000715)\end{array}$ & $\begin{array}{c}0.00428 * * * \\
(0.000662)\end{array}$ & $\begin{array}{c}0.00368 * * * \\
(0.000640)\end{array}$ & $\begin{array}{c}0.00252^{* *} \\
(0.00125)\end{array}$ \\
\hline Constant & $\begin{array}{c}0.0496 * * * \\
(0.0189)\end{array}$ & $\begin{array}{c}0.0614^{* * *} \\
(0.0165)\end{array}$ & $\begin{array}{c}0.0608 * * * \\
(0.0151)\end{array}$ & $\begin{array}{c}0.0566 * * * \\
(0.0140)\end{array}$ & $\begin{array}{c}0.0613^{* * *} \\
(0.0124)\end{array}$ & $\begin{array}{c}0.0670^{* * *} \\
(0.0115)\end{array}$ & $\begin{array}{c}0.0718^{* * *} \\
(0.0108)\end{array}$ & $\begin{array}{c}0.0714^{* * *} \\
(0.00996)\end{array}$ & $\begin{array}{c}0.0678^{* * *} \\
(0.00963)\end{array}$ & $\begin{array}{c}0.0496 * * * \\
(0.0189)\end{array}$ \\
\hline Observations & 252 & 252 & 252 & 252 & 252 & 252 & 252 & 252 & 252 & 252 \\
\hline R-squared & 0.043 & 0.077 & 0.090 & 0.109 & 0.144 & 0.170 & 0.204 & 0.215 & 0.183 & 0.043 \\
\hline & $\begin{array}{l}\text { (11) } \\
\text { GR11 }\end{array}$ & $\begin{array}{l}\text { (12) } \\
\text { GR12 }\end{array}$ & $\begin{array}{l}\text { (13) } \\
\text { GR13 }\end{array}$ & $\begin{array}{l}\text { (14) } \\
\text { GR14 }\end{array}$ & $\begin{array}{c}\text { (15) } \\
\text { GR15 }\end{array}$ & $\begin{array}{c}\text { (16) } \\
\text { GR16 }\end{array}$ & $\begin{array}{c}\text { (17) } \\
\text { GR17 }\end{array}$ & $\begin{array}{c}\text { (18) } \\
\text { GR18 }\end{array}$ & $\begin{array}{c}\text { (19) } \\
\text { GR19 }\end{array}$ & $\begin{array}{c}(20) \\
\text { GR20 }\end{array}$ \\
\hline EPI*NOFF & $\begin{array}{c}0.00198 \\
(0.00722)\end{array}$ & $\begin{array}{c}0.00127 \\
(0.00706)\end{array}$ & $\begin{array}{c}0.00369 \\
(0.00775)\end{array}$ & $\begin{array}{c}0.00310 \\
(0.00763)\end{array}$ & $\begin{array}{c}0.00659 \\
(0.00869)\end{array}$ & $\begin{array}{c}0.00162 \\
(0.00948)\end{array}$ & $\begin{array}{c}0.00273 \\
(0.00920)\end{array}$ & $\begin{array}{c}0.00110 \\
(0.00897)\end{array}$ & $\begin{array}{c}0.0166 \\
(0.0101)\end{array}$ & $\begin{array}{l}0.0181 * \\
(0.0100)\end{array}$ \\
\hline EPI*OFF & $\begin{array}{l}-0.00100 \\
(0.00677)\end{array}$ & $\begin{array}{l}-0.00687 \\
(0.00695)\end{array}$ & $\begin{array}{l}-0.00577 \\
(0.00647)\end{array}$ & $\begin{array}{l}-0.00511 \\
(0.00639)\end{array}$ & $\begin{array}{c}-0.00134 \\
(0.00651)\end{array}$ & $\begin{array}{l}-0.00291 \\
(0.00619)\end{array}$ & $\begin{array}{l}-0.00517 \\
(0.00600)\end{array}$ & $\begin{array}{l}-0.00620 \\
(0.00586)\end{array}$ & $\begin{array}{c}-0.00655 \\
(0.00544)\end{array}$ & $\begin{array}{c}-0.00842 \\
(0.00570)\end{array}$ \\
\hline OFF & $\begin{array}{c}-0.0134^{* * *} \\
(0.00374)\end{array}$ & $\begin{array}{c}-0.0122^{* * *} \\
(0.00366)\end{array}$ & $\begin{array}{c}-0.0122^{* * *} \\
(0.00342)\end{array}$ & $\begin{array}{c}-0.0124^{* * *} \\
(0.00339)\end{array}$ & $\begin{array}{c}-0.0142^{* * *} \\
(0.00417)\end{array}$ & $\begin{array}{c}-0.0139 * * * \\
(0.00396)\end{array}$ & $\begin{array}{c}-0.0151^{* * *} \\
(0.00384)\end{array}$ & $\begin{array}{c}-0.0144^{* * *} \\
(0.00382)\end{array}$ & $\begin{array}{c}-0.0145^{* * *} \\
(0.00349)\end{array}$ & $\begin{array}{c}-0.0138^{* * *} \\
(0.00345)\end{array}$ \\
\hline LY & $\begin{array}{c}-0.00784^{* * *} \\
(0.00134)\end{array}$ & $\begin{array}{c}-0.00826^{* * *} \\
(0.00132)\end{array}$ & $\begin{array}{c}-0.00813^{* * *} \\
(0.00124)\end{array}$ & $\begin{array}{c}-0.00847^{* * *} \\
(0.00122)\end{array}$ & $\begin{array}{c}-0.00641^{* * *} \\
(0.00164)\end{array}$ & $\begin{array}{c}-0.00681^{* * *} \\
(0.00157)\end{array}$ & $\begin{array}{c}-0.00719^{* * *} \\
(0.00153)\end{array}$ & $\begin{array}{c}-0.00707^{* * *} \\
(0.00152)\end{array}$ & $\begin{array}{c}-0.00735^{* * *} \\
(0.00140)\end{array}$ & $\begin{array}{c}-0.00755^{* * * *} \\
(0.00138)\end{array}$ \\
\hline iyr_sch & $\begin{array}{c}0.00341^{* * *} * \\
(0.000644)\end{array}$ & $\begin{array}{c}0.00360 * * * \\
(0.000630)\end{array}$ & $\begin{array}{c}0.00328^{* * *} * \\
(0.000600)\end{array}$ & $\begin{array}{l}0.00336 * * * \\
(0.000592)\end{array}$ & $\begin{array}{c}0.00301 * * * \\
(0.000770)\end{array}$ & $\begin{array}{l}0.00295 * * * \\
(0.000746)\end{array}$ & $\begin{array}{c}0.00289 * * * \\
(0.000731)\end{array}$ & $\begin{array}{c}0.00283^{* * *} * \\
(0.000716)\end{array}$ & $\begin{array}{c}0.00273^{* * *} \\
(0.000666)\end{array}$ & $\begin{array}{c}0.00273 * * * * \\
(0.000660)\end{array}$ \\
\hline Constant & $\begin{array}{l}0.0660 * * * \\
(0.00966)\end{array}$ & $\begin{array}{l}0.0671^{* * *} \\
(0.00948)\end{array}$ & $\begin{array}{l}0.0680^{* * *} \\
(0.00885)\end{array}$ & $\begin{array}{l}0.0703^{* * *} \\
(0.00872)\end{array}$ & $\begin{array}{c}0.0565^{* * *} \\
(0.0121)\end{array}$ & $\begin{array}{c}0.0610^{* * *} \\
(0.0115)\end{array}$ & $\begin{array}{c}0.0660^{* * *} \\
(0.0112)\end{array}$ & $\begin{array}{c}0.0661^{* * *} \\
(0.0112)\end{array}$ & $\begin{array}{c}0.0692^{* * *} \\
(0.0102)\end{array}$ & $\begin{array}{c}0.0695^{* * *} \\
(0.0101)\end{array}$ \\
\hline Observations & 246 & 245 & 241 & 240 & 165 & 162 & 160 & 157 & 158 & 158 \\
\hline R-squared & 0.171 & 0.185 & 0.188 & 0.200 & 0.179 & 0.178 & 0.203 & 0.201 & 0.233 & 0.237 \\
\hline
\end{tabular}

Standard errors in parentheses, *** $\mathrm{p}<0.01, * * \mathrm{p}<0.05, * \mathrm{p}<0.1$ 
Table A19: Evolution of growth post $10 \%$ episodes, controlling for education and saving rate, interaction with official flows dummy

\begin{tabular}{|c|c|c|c|c|c|c|c|c|c|c|}
\hline & $\begin{array}{c}(1) \\
\text { GR1 }\end{array}$ & $\begin{array}{c}(2) \\
\text { GR2 }\end{array}$ & $\begin{array}{c}\text { (3) } \\
\text { GR3 }\end{array}$ & $\begin{array}{c}\text { (4) } \\
\text { GR4 }\end{array}$ & $\begin{array}{c}\text { (5) } \\
\text { GR5 }\end{array}$ & $\begin{array}{c}6) \\
\text { GR6 }\end{array}$ & $\begin{array}{c}\text { (7) } \\
\text { GR7 }\end{array}$ & $\begin{array}{c}8) \\
\text { GR8 }\end{array}$ & $\begin{array}{c}9) \\
\text { GR9 }\end{array}$ & $\begin{array}{c}(10) \\
\text { GR10 }\end{array}$ \\
\hline EPI*NOFF & 0.0152 & 0.0113 & 0.00502 & -0.00742 & -0.00708 & -0.00764 & $\begin{array}{l}-0.00684 \\
\end{array}$ & -0.00387 & -0.00318 & -0.00899 \\
\hline & $(0.0188)$ & $(0.0166)$ & $(0.0151)$ & $(0.0139)$ & $(0.0121)$ & $(0.0113)$ & $(0.0106)$ & $(0.00983)$ & $(0.00956)$ & $(0.0107)$ \\
\hline EPI*OFF & -0.0179 & -0.00902 & -0.00496 & -0.00686 & -0.0108 & -0.00819 & -0.00734 & -0.00650 & -0.00616 & -0.00615 \\
\hline & $(0.0131)$ & $(0.0116)$ & $(0.0105)$ & (0.00973) & $(0.00848)$ & $(0.00791)$ & $(0.00744)$ & $(0.00686)$ & $(0.00668)$ & $(0.00734)$ \\
\hline OFF & $\begin{array}{c}-0.00419 \\
(0.00699)\end{array}$ & $\begin{array}{c}-0.00794 \\
(0.00617)\end{array}$ & $\begin{array}{c}-0.0136^{* *} \\
(0.00562)\end{array}$ & $\begin{array}{c}-0.0161^{* * *} \\
(0.00519)\end{array}$ & $\begin{array}{c}-0.0146^{* * * *} \\
(0.00452)\end{array}$ & $\begin{array}{c}-0.0147^{* * * *} \\
(0.00422)\end{array}$ & $\begin{array}{c}-0.0156^{* * *} \\
(0.00397)\end{array}$ & $\begin{array}{c}-0.0159 * * * \\
(0.00366)\end{array}$ & $\begin{array}{c}-0.0145^{* * *} \\
(0.00356)\end{array}$ & $\begin{array}{c}-0.0142 * * * \\
(0.00352)\end{array}$ \\
\hline LY & $\begin{array}{c}-0.00671^{* * * *} \\
(0.00247)\end{array}$ & $\begin{array}{c}-0.00797 * * * \\
(0.00218)\end{array}$ & $\begin{array}{c}-0.00858 * * * \\
(0.00199)\end{array}$ & $\begin{array}{c}-0.00800^{* * *} \\
(0.00183)\end{array}$ & $\begin{array}{c}-0.00872^{* * *} \\
(0.00160)\end{array}$ & $\begin{array}{c}-0.00886 * * * \\
(0.00149)\end{array}$ & $\begin{array}{c}-0.00913^{* * *} \\
(0.00140)\end{array}$ & $\begin{array}{c}-0.00896 * * * \\
(0.00129)\end{array}$ & $\begin{array}{c}-0.00814 * * * \\
(0.00126)\end{array}$ & $\begin{array}{c}-0.00790 * * * \\
(0.00125)\end{array}$ \\
\hline iyr_sch & $\begin{array}{c}0.00251^{* *} \\
(0.00119)\end{array}$ & $\begin{array}{c}0.00345 * * * \\
(0.00105)\end{array}$ & $\begin{array}{c}0.00383^{* * *} \\
(0.000960)\end{array}$ & $\begin{array}{c}0.00410 * * * \\
(0.000885)\end{array}$ & $\begin{array}{c}0.00442 * * * \\
(0.000771)\end{array}$ & $\begin{array}{c}0.00443^{* * *} \\
(0.000720)\end{array}$ & $\begin{array}{c}0.00455 * * * \\
(0.000677)\end{array}$ & $\begin{array}{c}0.00428 * * * \\
(0.000625)\end{array}$ & $\begin{array}{c}0.00362 * * * \\
(0.000608)\end{array}$ & $\begin{array}{c}0.00361^{* * *} \\
(0.000599)\end{array}$ \\
\hline Constant & $\begin{array}{c}0.0538 * * * \\
(0.0180)\end{array}$ & $\begin{array}{c}0.0579 * * * \\
(0.0159)\end{array}$ & $\begin{array}{c}0.0630 * * * \\
(0.0145)\end{array}$ & $\begin{array}{c}0.0601 * * * \\
(0.0133)\end{array}$ & $\begin{array}{c}0.0655 * * * \\
(0.0116)\end{array}$ & $\begin{array}{c}0.0687 * * * \\
(0.0109)\end{array}$ & $\begin{array}{c}0.0717^{* * *} \\
(0.0102)\end{array}$ & $\begin{array}{c}0.0718 * * * \\
(0.00942)\end{array}$ & $\begin{array}{c}0.0668 * * * \\
(0.00916)\end{array}$ & $\begin{array}{c}0.0653 * * * \\
(0.00912)\end{array}$ \\
\hline Observations & 260 & 260 & 260 & 260 & 260 & 260 & 260 & 260 & 260 & 257 \\
\hline R-squared & 0.042 & 0.066 & 0.097 & 0.132 & 0.176 & 0.193 & 0.225 & 0.239 & 0.201 & 0.201 \\
\hline & $\begin{array}{c}\text { (11) } \\
\text { GR11 }\end{array}$ & $\begin{array}{c}\text { (12) } \\
\text { GR12 }\end{array}$ & $\begin{array}{c}\text { (13) } \\
\text { GR13 }\end{array}$ & $\begin{array}{c}\text { (14) } \\
\text { GR14 }\end{array}$ & $\begin{array}{c}\text { (15) } \\
\text { GR15 }\end{array}$ & $\begin{array}{c}\text { (16) } \\
\text { GR16 }\end{array}$ & $\begin{array}{c}\text { (17) } \\
\text { GR17 }\end{array}$ & $\begin{array}{c}\text { (18) } \\
\text { GR18 }\end{array}$ & $\begin{array}{c}\text { (19) } \\
\text { GR19 }\end{array}$ & $\begin{array}{c}\text { (20) } \\
\text { GR20 }\end{array}$ \\
\hline EPI*NOFF & $\begin{array}{l}-0.00926 \\
(0.0106)\end{array}$ & $\begin{array}{c}-0.0185 \\
(0.0122)\end{array}$ & $\begin{array}{c}-0.0190 \\
(0.0121)\end{array}$ & $\begin{array}{l}-0.0186 \\
(0.0123)\end{array}$ & $\begin{array}{c}-0.0181 \\
(0.0116)\end{array}$ & $\begin{array}{l}-0.0161 \\
(0.0113)\end{array}$ & $\begin{array}{l}-0.0183^{*} \\
(0.0109)\end{array}$ & $\begin{array}{l}-0.00580 \\
(0.0145)\end{array}$ & $\begin{array}{l}-0.00324 \\
(0.0144)\end{array}$ & $\begin{array}{l}-0.00711 \\
(0.0143)\end{array}$ \\
\hline EPI*OFF & $\begin{array}{c}-0.00644 \\
(0.00726)\end{array}$ & $\begin{array}{l}-0.00501 \\
(0.00687)\end{array}$ & $\begin{array}{l}-0.00482 \\
(0.00679)\end{array}$ & $\begin{array}{l}0.000290 \\
(0.00696)\end{array}$ & $\begin{array}{c}-0.00141 \\
(0.00657)\end{array}$ & $\begin{array}{l}-0.00418 \\
(0.00636)\end{array}$ & $\begin{array}{l}-0.00528 \\
(0.00619)\end{array}$ & $\begin{array}{l}-0.00507 \\
(0.00583)\end{array}$ & $\begin{array}{l}-0.00751 \\
(0.00618)\end{array}$ & $\begin{array}{c}-0.00382 \\
(0.00666)\end{array}$ \\
\hline OFF & $\begin{array}{c}-0.0127^{* * *} \\
(0.00348)\end{array}$ & $\begin{array}{c}-0.0130^{* * *} \\
(0.00332)\end{array}$ & $\begin{array}{c}-0.0131^{* * *} \\
(0.00329)\end{array}$ & $\begin{array}{c}-0.0148^{* * *} \\
(0.00410)\end{array}$ & $\begin{array}{c}-0.0144^{* * *} \\
(0.00388)\end{array}$ & $\begin{array}{c}-0.0155^{* * *} \\
(0.00376)\end{array}$ & $\begin{array}{c}-0.0148^{* * *} \\
(0.00373)\end{array}$ & $\begin{array}{c}-0.0146^{* * *} \\
(0.00346)\end{array}$ & $\begin{array}{c}-0.0140^{* * *} \\
(0.00342)\end{array}$ & $\begin{array}{c}-0.0149 * * * \\
(0.00340)\end{array}$ \\
\hline LY & $\begin{array}{c}-0.00814^{* * * *} \\
(0.00123)\end{array}$ & $\begin{array}{c}-0.00793^{* * *} \\
(0.00117)\end{array}$ & $\begin{array}{c}-0.00841^{* * *} \\
(0.00116)\end{array}$ & $\begin{array}{c}-0.00569 * * * \\
(0.00162)\end{array}$ & $\begin{array}{c}-0.00634^{* * * *} \\
(0.00154)\end{array}$ & $\begin{array}{c}-0.00673^{* * *} \\
(0.00150)\end{array}$ & $\begin{array}{c}-0.00665^{* * *} \\
(0.00148)\end{array}$ & $\begin{array}{c}-0.00663^{* * *} \\
(0.00137)\end{array}$ & $\begin{array}{c}-0.00686^{* * * *} \\
(0.00136)\end{array}$ & $\begin{array}{c}-0.00701^{* * * *} \\
(0.00135)\end{array}$ \\
\hline iyr_sch & $\begin{array}{c}0.00377^{* * *} * \\
(0.000592)\end{array}$ & $\begin{array}{c}0.00335^{* * *} \\
(0.000569)\end{array}$ & $\begin{array}{c}0.00344 * * * \\
(0.000562)\end{array}$ & $\begin{array}{c}0.00286 * * * * \\
(0.000777)\end{array}$ & $\begin{array}{c}0.00296 * * * \\
(0.000740)\end{array}$ & $\begin{array}{c}0.00290 * * * \\
(0.000723)\end{array}$ & $\begin{array}{c}0.00286 * * * \\
(0.000706)\end{array}$ & $\begin{array}{c}0.00270 * * * \\
(0.000666)\end{array}$ & $\begin{array}{c}0.00270 * * * * \\
(0.000661)\end{array}$ & $\begin{array}{c}0.00266 * * * \\
(0.000657)\end{array}$ \\
\hline Constant & $\begin{array}{l}0.0650 * * * \\
(0.00902)\end{array}$ & $\begin{array}{l}0.0659 * * * \\
(0.00853)\end{array}$ & $\begin{array}{l}0.0692 * * * \\
(0.00843)\end{array}$ & $\begin{array}{c}0.0511^{* * *} \\
(0.0119)\end{array}$ & $\begin{array}{c}0.0569 * * * \\
(0.0113)\end{array}$ & $\begin{array}{c}0.0619 * * * \\
(0.0109)\end{array}$ & $\begin{array}{c}0.0621^{* * *} \\
(0.0109)\end{array}$ & $\begin{array}{l}0.0629 * * * \\
(0.01000)\end{array}$ & $\begin{array}{l}0.0636 * * * \\
(0.00989)\end{array}$ & $\begin{array}{l}0.0658 * * * \\
(0.00983)\end{array}$ \\
\hline Observations & 257 & 253 & 251 & 169 & 167 & 165 & 162 & 164 & 164 & 163 \\
\hline R-squared & 0.203 & 0.202 & 0.217 & 0.181 & 0.191 & 0.211 & 0.215 & 0.214 & 0.213 & 0.213 \\
\hline
\end{tabular}

Standard errors in parentheses, *** $\mathrm{p}<0.01, * * \mathrm{p}<0.05, * \mathrm{p}<0.1$ 
Table A20: Evolution of growth post 4\% episodes, controlling for education and saving rate, interaction with FDI dummy

\begin{tabular}{|c|c|c|c|c|c|c|c|c|c|c|}
\hline & (1) & (2) & (3) & (4) & (5) & (6) & (7) & (8) & (9) & (10) \\
\hline & GR1 & GR2 & GR3 & GR4 & GR5 & GR6 & GR7 & GR8 & GR9 & GR10 \\
\hline \multirow[t]{2}{*}{ EPI*NFDI } & 0.00909 & 0.000753 & -0.00241 & $-0.0113^{*}$ & $-0.0101^{*}$ & $-0.0118^{* *}$ & $-0.0137 * * *$ & $-0.0126^{* * *}$ & $-0.0127 * * *$ & $-0.0140 * * *$ \\
\hline & $(0.00843)$ & $(0.00764)$ & $(0.00696)$ & $(0.00619)$ & $(0.00564)$ & $(0.00525)$ & $(0.00498)$ & $(0.00471)$ & $(0.00449)$ & $(0.00436)$ \\
\hline \multirow[t]{2}{*}{ EPI*FDI } & $0.0229 * * *$ & $0.0123 *$ & 0.0103 & 0.00622 & 0.00355 & $6.50 \mathrm{e}-05$ & -0.000481 & 0.000742 & 0.00221 & -0.000448 \\
\hline & $(0.00778)$ & $(0.00705)$ & $(0.00642)$ & $(0.00571)$ & $(0.00520)$ & $(0.00484)$ & $(0.00459)$ & $(0.00435)$ & $(0.00414)$ & $(0.00420)$ \\
\hline \multirow[t]{2}{*}{ FDI } & 0.00537 & 0.00916 & $0.00956 *$ & $0.00945^{* *}$ & $0.00908 * *$ & $0.00822^{* *}$ & $0.00822^{* *}$ & $0.00691^{*}$ & 0.00427 & $0.00552 *$ \\
\hline & $(0.00653)$ & $(0.00591)$ & $(0.00539)$ & $(0.00479)$ & $(0.00436)$ & $(0.00407)$ & $(0.00386)$ & $(0.00365)$ & $(0.00348)$ & $(0.00330)$ \\
\hline LY & $\begin{array}{c}-0.00579 * * \\
(0.00274)\end{array}$ & $\begin{array}{c}-0.00689 * * * \\
(0.00248)\end{array}$ & $\begin{array}{c}-0.00604^{* * *} \\
(0.00226)\end{array}$ & $\begin{array}{c}-0.00593^{* * *} * \\
(0.00201)\end{array}$ & $\begin{array}{c}-0.00585^{* * *} \\
(0.00183)\end{array}$ & $\begin{array}{c}-0.00661 * * * \\
(0.00171)\end{array}$ & $\begin{array}{c}-0.00673^{* * *} \\
(0.00162)\end{array}$ & $\begin{array}{c}-0.00660^{* * *} \\
(0.00153)\end{array}$ & $\begin{array}{c}-0.00626^{* * *} \\
(0.00146)\end{array}$ & $\begin{array}{c}-0.00586^{* * *} \\
(0.00144)\end{array}$ \\
\hline \multirow[t]{2}{*}{ iyr_sch } & $0.00313^{* *}$ & $0.00345^{* * *}$ & $0.00286^{* *}$ & $0.00320^{* * *}$ & $0.00342^{* * *}$ & $0.00360 * * *$ & $0.00369 * * *$ & $0.00352 * * *$ & $0.00309^{* * *}$ & $0.00287^{* * *}$ \\
\hline & $(0.00144)$ & $(0.00131)$ & $(0.00119)$ & $(0.00106)$ & $(0.000965)$ & $(0.000899)$ & $(0.000853)$ & $(0.000807)$ & $(0.000769)$ & $(0.000746)$ \\
\hline \multirow[t]{2}{*}{ Constant } & $0.0385 * *$ & $0.0438^{* * *}$ & $0.0425^{* * *}$ & $0.0436^{* * *}$ & $0.0427 * * *$ & $0.0509 * * *$ & $0.0527 * * *$ & $0.0528 * * *$ & $0.0516^{* * *}$ & $0.0497 * * *$ \\
\hline & $(0.0185)$ & $(0.0167)$ & $(0.0152)$ & $(0.0135)$ & $(0.0123)$ & $(0.0115)$ & $(0.0109)$ & $(0.0103)$ & $(0.00983)$ & $(0.00973)$ \\
\hline Observations & 207 & 207 & 207 & 207 & 207 & 207 & 207 & 207 & 207 & 196 \\
\hline \multirow[t]{3}{*}{ R-squared } & 0.123 & 0.127 & 0.129 & 0.168 & 0.178 & 0.189 & 0.218 & 0.214 & 0.191 & 0.195 \\
\hline & (11) & (12) & (13) & (14) & (15) & (16) & $(17)$ & (18) & (19) & (20) \\
\hline & GR11 & GR12 & GR13 & GR14 & GR15 & GR16 & GR17 & GR18 & GR19 & GR20 \\
\hline \multirow[t]{2}{*}{ EPI*NFDI } & $-0.0126^{* * *}$ & $-0.0106^{* *}$ & $-0.0102 * *$ & $-0.00948^{* *}$ & $-0.00882 *$ & $-0.00997^{* *}$ & $-0.00933^{* *}$ & $-0.00994 * *$ & $-0.00899 * *$ & $-0.00883^{* *}$ \\
\hline & $(0.00430)$ & $(0.00415)$ & $(0.00410)$ & $(0.00450)$ & $(0.00451)$ & $(0.00455)$ & $(0.00448)$ & $(0.00434)$ & $(0.00437)$ & $(0.00431)$ \\
\hline \multirow[t]{2}{*}{ EPI*FDI } & -0.000166 & -0.00113 & -0.000292 & -0.00182 & -0.00287 & -0.00422 & -0.00472 & -0.00417 & -0.000459 & 0.00129 \\
\hline & $(0.00436)$ & $(0.00414)$ & $(0.00416)$ & $(0.00541)$ & $(0.00555)$ & $(0.00553)$ & $(0.00549)$ & $(0.00533)$ & $(0.00557)$ & $(0.00567)$ \\
\hline \multirow[t]{2}{*}{ FDI } & 0.00338 & 0.00425 & 0.00410 & $0.00710^{*}$ & $0.00780^{*}$ & $0.00782^{*}$ & $0.00794^{* *}$ & 0.00613 & 0.00401 & 0.00372 \\
\hline & $(0.00329)$ & $(0.00309)$ & $(0.00305)$ & $(0.00427)$ & $(0.00417)$ & $(0.00411)$ & $(0.00401)$ & $(0.00388)$ & $(0.00391)$ & $(0.00384)$ \\
\hline LY & $-0.00660 * * *$ & $-0.00643^{* * *}$ & $-0.00667^{* * * *}$ & $\begin{array}{c}-0.00378^{* *} \\
0.0018 ?\end{array}$ & $-0.00433^{* * *}$ & $-0.00493^{* * *}$ & $-0.00480^{* * *}$ & $-0.00509 * * *$ & $-0.00552^{* * *}$ & $-0.00535 * * *$ \\
\hline \multirow[t]{2}{*}{ iyr_sch } & $0.00339 * * *$ & $0.00293 * * *$ & $0.00297 * * *$ & $0.00221^{* *}$ & $0.00246^{* * * *}$ & $0.00266^{* * *}$ & $0.00265 * * *$ & $0.00248^{* * * *}$ & $0.00271^{* * *}$ & $0.00250^{* * *}$ \\
\hline & $(0.000753)$ & $(0.000720)$ & $(0.000711)$ & $(0.000904)$ & $(0.000921)$ & $(0.000926)$ & $(0.000903)$ & $(0.000879)$ & $(0.000904)$ & $(0.000896)$ \\
\hline \multirow[t]{2}{*}{ Constant } & $0.0524^{* * *}$ & $0.0535^{* * *}$ & $0.0551^{* * *}$ & $0.0355^{* * *}$ & $0.0389 * * *$ & $0.0440^{* * *}$ & $0.0435^{* * *}$ & $0.0480^{* * *}$ & $0.0497 * * *$ & $0.0503^{* * *}$ \\
\hline & $(0.00978)$ & $(0.00919)$ & $(0.00912)$ & $(0.0124)$ & $(0.0122)$ & $(0.0121)$ & $(0.0120)$ & $(0.0115)$ & $(0.0116)$ & $(0.0116)$ \\
\hline Observations & 194 & 189 & 188 & 135 & 129 & 125 & 123 & 123 & 121 & 119 \\
\hline R-squared & 0.194 & 0.182 & 0.192 & 0.151 & 0.159 & 0.169 & 0.171 & 0.158 & 0.148 & 0.144 \\
\hline
\end{tabular}

Standard errors in parentheses, *** $\mathrm{p}<0.01,{ }^{* *} \mathrm{p}<0.05,{ }^{*} \mathrm{p}<0.1$ 
Table A21: Evolution of growth post 6\% episodes, controlling for education and saving rate, interaction with FDI dummy

\begin{tabular}{|c|c|c|c|c|c|c|c|c|c|c|}
\hline & (1) & (2) & (3) & (4) & (5) & (6) & (7) & (8) & (9) & (10) \\
\hline & GR1 & GR2 & GR3 & GR4 & GR5 & GR6 & GR7 & GR8 & GR9 & GR10 \\
\hline EPI*NFDI & $\begin{array}{l}0.00755 \\
(0.0114)\end{array}$ & -0.000175 & -0.000992 & $\begin{array}{c}-0.00941 \\
(0.00841)\end{array}$ & $\begin{array}{c}-0.0116 \\
(0.00734)\end{array}$ & $\begin{array}{c}-0.0129 * \\
(0.00677)\end{array}$ & $-0.0146 * *$ & $\begin{array}{c}-0.0133^{* *} \\
0.00591\end{array}$ & $\begin{array}{c}-0.0128^{* *} \\
0.00568\end{array}$ & $-0.0128^{* *}$ \\
\hline EPI*FDI & $0.0189^{* *}$ & 0.0107 & 0.0103 & 0.00785 & 0.00500 & 0.00265 & 0.00330 & 0.00430 & 0.00463 & 0.00297 \\
\hline FDI & $\begin{array}{c}(0.00912) \\
0.00615\end{array}$ & $\begin{array}{l}(0.00796) \\
0.00835 *\end{array}$ & $\begin{array}{l}(0.00721) \\
0.00933^{* *}\end{array}$ & $\begin{array}{l}(0.00673) \\
0.00988^{* *}\end{array}$ & $\begin{array}{l}(0.00587) \\
0.00843^{* *}\end{array}$ & $\begin{array}{l}(0.00542) \\
0.00735^{* *}\end{array}$ & $\begin{array}{l}(0.00505) \\
0.00729 * *\end{array}$ & $\begin{array}{l}(0.00473) \\
0.00605^{* *}\end{array}$ & $\begin{array}{c}(0.00454) \\
0.00422\end{array}$ & $\begin{array}{l}(0.00507) \\
0.00530^{*}\end{array}$ \\
\hline LY & $\begin{array}{c}-0.00578^{* *} \\
(0.00266)\end{array}$ & $\begin{array}{c}-0.00777^{* * *} \\
(0.00232)\end{array}$ & $\begin{array}{c}-0.00668^{* * * *} \\
(0.00210)\end{array}$ & $\begin{array}{c}-0.00546 * * * \\
(0.00196)\end{array}$ & $\begin{array}{c}-0.00628^{* * * *} \\
(0.00171)\end{array}$ & $\begin{array}{c}-0.00688^{* * *} \\
(0.00158)\end{array}$ & $\begin{array}{c}-0.00682 * * * \\
(0.00147)\end{array}$ & $\begin{array}{c}-0.00661^{* * *} \\
(0.00138)\end{array}$ & $\begin{array}{c}-0.00626 * * * \\
(0.00132)\end{array}$ & $\begin{array}{c}-0.00608^{* * *} \\
(0.00133)\end{array}$ \\
\hline iyr_sch & $\begin{array}{c}0.00294^{* *} \\
(0.00139)\end{array}$ & $\begin{array}{c}0.00380^{* * *} \\
(0.00121)\end{array}$ & $\begin{array}{c}0.00342 * * * \\
(0.00110)\end{array}$ & $\begin{array}{c}0.00365^{* * *} * \\
(0.00102)\end{array}$ & $\begin{array}{c}0.00404^{* * *} \\
(0.000893)\end{array}$ & $\begin{array}{c}0.00416 * * * \\
(0.000824)\end{array}$ & $\begin{array}{c}0.00419 * * * \\
(0.000768)\end{array}$ & $\begin{array}{c}0.00403^{* * *} \\
(0.000719)\end{array}$ & $\begin{array}{c}0.00354 * * * \\
(0.000691)\end{array}$ & $\begin{array}{c}0.00339 * * * \\
(0.000690)\end{array}$ \\
\hline Constant & $\begin{array}{l}0.0374 * * \\
(0.0174)\end{array}$ & $\begin{array}{c}0.0488 * * * \\
(0.0152)\end{array}$ & $\begin{array}{c}0.0435 * * * \\
(0.0137)\end{array}$ & $\begin{array}{c}0.0341 * * * \\
(0.0128)\end{array}$ & $\begin{array}{c}0.0411^{* * *} \\
(0.0112)\end{array}$ & $\begin{array}{c}0.0482 * * * \\
(0.0103)\end{array}$ & $\begin{array}{c}0.0490 * * * \\
(0.00962)\end{array}$ & $\begin{array}{c}0.0482 * * * \\
(0.00900)\end{array}$ & $\begin{array}{c}0.0472 * * * \\
(0.00865)\end{array}$ & $\begin{array}{c}0.0466 * * * \\
(0.00870)\end{array}$ \\
\hline Observations & 230 & 230 & 230 & 230 & 230 & 230 & 230 & 230 & 230 & 222 \\
\hline R-squared & 0.070 & 0.103 & 0.114 & 0.138 & 0.172 & 0.189 & 0.219 & 0.220 & 0.187 & 0.182 \\
\hline & $\begin{array}{c}\text { (11) } \\
\text { GR11 }\end{array}$ & $\begin{array}{c}\text { (12) } \\
\text { GR12 }\end{array}$ & $\begin{array}{c}\text { (13) } \\
\text { GR13 }\end{array}$ & $\begin{array}{c}\text { (14) } \\
\text { GR14 }\end{array}$ & $\begin{array}{c}\text { (15) } \\
\text { GR15 }\end{array}$ & $\begin{array}{c}\text { (16) } \\
\text { GR16 }\end{array}$ & $\begin{array}{c}\text { (17) } \\
\text { GR17 }\end{array}$ & $\begin{array}{c}\text { (18) } \\
\text { GR18 }\end{array}$ & $\begin{array}{c}\text { (19) } \\
\text { GR19 }\end{array}$ & $\begin{array}{c}(20) \\
\text { GR20 }\end{array}$ \\
\hline EPI*NFDI & $\begin{array}{c}-0.0115^{* *} \\
0.00567\end{array}$ & $\begin{array}{c}-0.00977^{*} \\
(0.00552)\end{array}$ & $\begin{array}{c}-0.00895 \\
(0.00547)\end{array}$ & $\begin{array}{l}-0.00479 \\
(0.00582)\end{array}$ & $\begin{array}{c}-0.00629 \\
(0.00554)\end{array}$ & $\begin{array}{c}-0.00713 \\
(0.00547)\end{array}$ & $\begin{array}{c}-0.00686 \\
(0.00530)\end{array}$ & $\begin{array}{l}-0.00664 \\
(0.00504)\end{array}$ & $\begin{array}{c}-0.00622 \\
(0.00502)\end{array}$ & $\begin{array}{c}-0.00609 \\
(0.00496)\end{array}$ \\
\hline EPI*FDI & $\begin{array}{l}0.000686 \\
(0.00515)\end{array}$ & $\begin{array}{r}-0.000717 \\
(0.00496)\end{array}$ & $\begin{array}{c}0.00109 \\
(0.00508)\end{array}$ & $\begin{array}{l}0.000890 \\
(0.00607)\end{array}$ & $\begin{array}{l}-0.00252 \\
(0.00599)\end{array}$ & $\begin{array}{c}-0.00292 \\
(0.00617)\end{array}$ & $\begin{array}{l}-0.00489 \\
(0.00600)\end{array}$ & $\begin{array}{l}-0.00424 \\
(0.00567)\end{array}$ & $\begin{array}{l}-0.00214 \\
(0.00593)\end{array}$ & $\begin{array}{l}0.000999 \\
(0.00617)\end{array}$ \\
\hline FDI & $\begin{array}{c}0.00381 \\
0.00277)\end{array}$ & $\begin{array}{c}0.00402 \\
(0.00260)\end{array}$ & $\begin{array}{c}0.00414 \\
(0.00257)\end{array}$ & $0.00619 *$ & $0.00597 *$ & $0.00574 *$ & $0.00666 * *$ & $0.00517^{*}$ & $\begin{array}{c}0.00404 \\
(0.00299)\end{array}$ & $\begin{array}{c}0.00386 \\
(0.00295)\end{array}$ \\
\hline LY & $-0.00663^{* * *}$ & $-0.00659 * * *$ & $-0.00676^{* * *}$ & $\begin{array}{c}-0.00378^{* *} \\
(0.00169)\end{array}$ & $-0.00440^{* * *}$ & $-0.00496 * * *$ & $-0.00482^{* * *}$ & $-0.00509^{* * *}$ & $-0.00539 * * *$ & $-0.00528 * * *$ \\
\hline iyr_sch & $\begin{array}{c}0.00370 * * * \\
(0.000686)\end{array}$ & $\begin{array}{l}0.00336 * * * \\
(0.000654)\end{array}$ & $\begin{array}{c}0.00337^{* * *} \\
(0.000648)\end{array}$ & $\begin{array}{c}0.00299 * * * \\
(0.000871)\end{array}$ & $\begin{array}{c}0.00297 * * * \\
(0.000841)\end{array}$ & $\begin{array}{c}0.00318^{* * *} \\
(0.000853)\end{array}$ & $\begin{array}{c}0.00305^{* * *} \\
(0.000832)\end{array}$ & $\begin{array}{c}0.00303^{* * *} \\
(0.000786)\end{array}$ & $\begin{array}{c}0.00305^{* * *} \\
(0.000788)\end{array}$ & $\begin{array}{c}0.00290 * * * \\
(0.000779)\end{array}$ \\
\hline Constant & $0.0489 * * *$ & $0.0507 * * *$ & $0.0517^{* * *}$ & $0.0290 * * *$ & $0.0354 * * *$ & $0.0401^{* * *}$ & $0.0402^{* * *}$ & $0.0432 * * *$ & $0.0450 * * *$ & $0.0456 * * *$ \\
\hline Observations & 221 & 217 & 216 & 146 & 143 & 140 & 137 & 139 & 139 & 138 \\
\hline R-squared & 0.183 & 0.177 & 0.184 & 0.142 & 0.140 & 0.150 & 0.163 & 0.154 & 0.143 & 0.139 \\
\hline
\end{tabular}

Standard errors in parentheses, *** $\mathrm{p}<0.01, * * \mathrm{p}<0.05, * \mathrm{p}<0.1$ 
Table A22: Evolution of growth post $8 \%$ episodes, controlling for education and saving rate, interaction with FDI dummy

\begin{tabular}{|c|c|c|c|c|c|c|c|c|c|c|}
\hline & (1) & (2) & (3) & (4) & (5) & (6) & (7) & (8) & (9) & (10) \\
\hline & GR1 & GR2 & GR3 & GR4 & GR5 & GR6 & GR7 & GR8 & GR9 & GR10 \\
\hline EPI*NFDI & $\begin{array}{c}0.0119 \\
(0.0157)\end{array}$ & $\begin{array}{c}0.0134 \\
(0.0137)\end{array}$ & $\begin{array}{c}0.0141 \\
(0.0125)\end{array}$ & $\begin{array}{l}0.00568 \\
(0.0116)\end{array}$ & $\begin{array}{l}0.00161 \\
(0.0103)\end{array}$ & $\begin{array}{c}-0.00150 \\
(0.00957)\end{array}$ & $\begin{array}{c}-0.00668 \\
(0.00902)\end{array}$ & $\begin{array}{c}-0.00684 \\
(0.00841)\end{array}$ & $\begin{array}{c}-0.00868 \\
(0.00813)\end{array}$ & $\begin{array}{c}-0.0103 \\
(0.00865)\end{array}$ \\
\hline EPI*FDI & $\begin{array}{l}0.00957 \\
(0.0102)\end{array}$ & $\begin{array}{c}0.00287 \\
(0.00890)\end{array}$ & $\begin{array}{c}0.00341 \\
(0.00813)\end{array}$ & $\begin{array}{l}0.000627 \\
(0.00754)\end{array}$ & $\begin{array}{c}-0.000730 \\
(0.00668)\end{array}$ & $\begin{array}{l}-0.00167 \\
(0.00620)\end{array}$ & $\begin{array}{c}-0.000197 \\
(0.00584)\end{array}$ & $\begin{array}{c}0.00148 \\
(0.00544)\end{array}$ & $\begin{array}{c}0.00151 \\
(0.00527)\end{array}$ & $\begin{array}{c}-0.00174 \\
(0.00601)\end{array}$ \\
\hline FDI & $\begin{array}{c}0.00642 \\
(0.00515)\end{array}$ & $\begin{array}{l}0.00794^{*} \\
(0.00449)\end{array}$ & $\begin{array}{c}0.0112 * * * \\
(0.00410)\end{array}$ & $\begin{array}{c}0.0118 * * * \\
(0.00381)\end{array}$ & $\begin{array}{c}0.0107 * * * \\
(0.00337)\end{array}$ & $\begin{array}{c}0.00965 * * * \\
(0.00313)\end{array}$ & $\begin{array}{c}0.00931 * * * \\
(0.00295)\end{array}$ & $\begin{array}{c}0.00828 * * * \\
(0.00275)\end{array}$ & $\begin{array}{c}0.00666 * * \\
(0.00266)\end{array}$ & $\begin{array}{c}0.00748^{* * * *} \\
(0.00260)\end{array}$ \\
\hline LY & $\begin{array}{c}-0.00555^{* *} \\
(0.00244)\end{array}$ & $\begin{array}{c}-0.00740^{* * * *} \\
(0.00213)\end{array}$ & $\begin{array}{c}-0.00606^{* * * *} \\
(0.00195)\end{array}$ & $\begin{array}{c}-0.00496 * * * \\
(0.00181)\end{array}$ & $\begin{array}{c}-0.00578 * * * \\
(0.00160)\end{array}$ & $\begin{array}{c}-0.00627^{* * *} \\
(0.00148)\end{array}$ & $\begin{array}{c}-0.00653^{* * * *} \\
(0.00140)\end{array}$ & $\begin{array}{c}-0.00626 * * * \\
(0.00130)\end{array}$ & $\begin{array}{c}-0.00588^{* * * *} \\
(0.00126)\end{array}$ & $\begin{array}{c}-0.00546^{* * *} \\
(0.00126)\end{array}$ \\
\hline iyr_sch & $\begin{array}{c}0.00218^{*} \\
(0.00129)\end{array}$ & $\begin{array}{c}0.00348 * * * \\
(0.00113)\end{array}$ & $\begin{array}{c}0.00336 * * * \\
(0.00103)\end{array}$ & $\begin{array}{c}0.00355^{* * *} \\
(0.000956)\end{array}$ & $\begin{array}{c}0.00393 * * * \\
(0.000846)\end{array}$ & $\begin{array}{c}0.00409 * * * \\
(0.000785)\end{array}$ & $\begin{array}{c}0.00426^{* * *} \\
(0.000740)\end{array}$ & $\begin{array}{c}0.00399 * * * \\
(0.000690)\end{array}$ & $\begin{array}{c}0.00346 * * * \\
(0.000668)\end{array}$ & $\begin{array}{c}0.00319 * * * \\
(0.000665)\end{array}$ \\
\hline Constant & $\begin{array}{c}0.0422 * * * \\
(0.0156)\end{array}$ & $\begin{array}{c}0.0479 * * * \\
(0.0136)\end{array}$ & $\begin{array}{c}0.0367 * * * \\
(0.0124)\end{array}$ & $\begin{array}{l}0.0290^{* *} \\
(0.0115)\end{array}$ & $\begin{array}{c}0.0357 * * * \\
(0.0102)\end{array}$ & $\begin{array}{c}0.0413^{* * *} \\
(0.00946)\end{array}$ & $\begin{array}{c}0.0439 * * * \\
(0.00892)\end{array}$ & $\begin{array}{c}0.0435^{* * *} \\
(0.00831)\end{array}$ & $\begin{array}{c}0.0425^{* * *} \\
(0.00804)\end{array}$ & $\begin{array}{c}0.0408 * * * \\
(0.00800)\end{array}$ \\
\hline $\begin{array}{l}\text { Observations } \\
\text { R-squared }\end{array}$ & $\begin{array}{c}252 \\
0.042\end{array}$ & $\begin{array}{c}252 \\
0.077\end{array}$ & $\begin{array}{c}252 \\
0.099\end{array}$ & $\begin{array}{c}252 \\
0.119\end{array}$ & $\begin{array}{c}252 \\
0.151\end{array}$ & $\begin{array}{c}252 \\
0.169\end{array}$ & $\begin{array}{c}252 \\
0.197\end{array}$ & $\begin{array}{c}252 \\
0.197\end{array}$ & $\begin{array}{c}252 \\
0.164\end{array}$ & $\begin{array}{c}246 \\
0.159\end{array}$ \\
\hline & $\begin{array}{l}\text { (11) } \\
\text { GR11 }\end{array}$ & $\begin{array}{l}\text { (12) } \\
\text { GR12 }\end{array}$ & $\begin{array}{c}\text { (13) } \\
\text { GR13 }\end{array}$ & $\begin{array}{l}\text { (14) } \\
\text { GR14 }\end{array}$ & $\begin{array}{l}\text { (15) } \\
\text { GR15 }\end{array}$ & $\begin{array}{c}\text { (16) } \\
\text { GR16 }\end{array}$ & $\begin{array}{l}\text { (17) } \\
\text { GR17 }\end{array}$ & $\begin{array}{c}18) \\
\text { GR18 }\end{array}$ & $\begin{array}{l}\text { (19) } \\
\text { GR19 }\end{array}$ & $\begin{array}{c}\text { (20) } \\
\text { GR20 }\end{array}$ \\
\hline EPI*NFDI & $\begin{array}{c}-0.0110 \\
(0.00851)\end{array}$ & $\begin{array}{c}-0.00582 \\
(0.00893)\end{array}$ & $\begin{array}{c}-0.00556 \\
(0.00880)\end{array}$ & $\begin{array}{c}-0.00183 \\
(0.00897)\end{array}$ & $\begin{array}{c}-0.00318 \\
(0.00851)\end{array}$ & $\begin{array}{c}-0.00483 \\
(0.00837)\end{array}$ & $\begin{array}{c}-0.00560 \\
(0.00810)\end{array}$ & $\begin{array}{c}-0.00593 \\
(0.00775)\end{array}$ & $\begin{array}{c}-0.00643 \\
(0.00772)\end{array}$ & $\begin{array}{c}-0.00521 \\
(0.00768)\end{array}$ \\
\hline EPI*FDI & $\begin{array}{l}-0.00414 \\
(0.00616)\end{array}$ & $\begin{array}{l}-0.00634 \\
(0.00608)\end{array}$ & $\begin{array}{l}-0.00638 \\
(0.00600)\end{array}$ & $\begin{array}{c}-0.00372 \\
(0.00664)\end{array}$ & $\begin{array}{l}-0.00812 \\
(0.00668)\end{array}$ & $\begin{array}{c}-0.00960 \\
(0.00657)\end{array}$ & $\begin{array}{l}-0.0110^{*} \\
(0.00638)\end{array}$ & $\begin{array}{c}-0.00758 \\
(0.00650)\end{array}$ & $\begin{array}{l}-0.00680 \\
(0.00702)\end{array}$ & $\begin{array}{l}-0.00211 \\
(0.00770)\end{array}$ \\
\hline FDI & $\begin{array}{c}0.00605^{* *} \\
(0.00256)\end{array}$ & $\begin{array}{c}0.00611^{* *} \\
(0.00240)\end{array}$ & $\begin{array}{c}0.00615^{* *} \\
(0.00237)\end{array}$ & $\begin{array}{c}0.00822 * * * \\
(0.00299)\end{array}$ & $\begin{array}{c}0.00796 * * * \\
(0.00284)\end{array}$ & $\begin{array}{c}0.00775^{* * *} \\
(0.00280)\end{array}$ & $\begin{array}{c}0.00820 * * * \\
(0.00276)\end{array}$ & $\begin{array}{c}0.00677 * * * \\
(0.00260)\end{array}$ & $\begin{array}{c}0.00543 * * \\
(0.00258)\end{array}$ & $\begin{array}{c}0.00516^{* *} \\
(0.00257)\end{array}$ \\
\hline LY & $\begin{array}{c}-0.00601 * * * \\
(0.00124)\end{array}$ & $\begin{array}{c}-0.00592^{* * *} \\
(0.00117)\end{array}$ & $\begin{array}{c}-0.00628 * * * \\
(0.00116)\end{array}$ & $\begin{array}{c}-0.00335^{* *} \\
(0.00149)\end{array}$ & $\begin{array}{c}-0.00384^{* * *} \\
(0.00143)\end{array}$ & $\begin{array}{c}-0.00402^{* * *} \\
(0.00142)\end{array}$ & $\begin{array}{c}-0.00389 * * * \\
(0.00139)\end{array}$ & $\begin{array}{c}-0.00414 * * * \\
(0.00132)\end{array}$ & $\begin{array}{c}-0.00454 * * * \\
(0.00131)\end{array}$ & $\begin{array}{c}-0.00455^{* * *} \\
(0.00131)\end{array}$ \\
\hline iyr_sch & $\begin{array}{l}0.00343 * * * \\
(0.000657)\end{array}$ & $\begin{array}{c}0.00312 * * * \\
(0.000629)\end{array}$ & $\begin{array}{c}0.00321^{* * *} \\
(0.000620)\end{array}$ & $\begin{array}{c}0.00293 * * * \\
(0.000793)\end{array}$ & $\begin{array}{c}0.00285 * * * \\
(0.000765)\end{array}$ & $\begin{array}{c}0.00284 * * * \\
(0.000759)\end{array}$ & $\begin{array}{c}0.00270 * * * \\
(0.000740)\end{array}$ & $\begin{array}{c}0.00267 * * * \\
(0.000706)\end{array}$ & $\begin{array}{c}0.00274 * * * \\
(0.000704)\end{array}$ & $\begin{array}{c}0.00268 * * * \\
(0.000702)\end{array}$ \\
\hline Constant & $\begin{array}{c}0.0436 * * * \\
(0.00786)\end{array}$ & $\begin{array}{c}0.0447 * * * \\
(0.00739)\end{array}$ & $\begin{array}{c}0.0470 * * * \\
(0.00730)\end{array}$ & $\begin{array}{l}0.0243 * * \\
(0.00937)\end{array}$ & $\begin{array}{c}0.0299 * * * \\
(0.00896)\end{array}$ & $\begin{array}{c}0.0326 * * * \\
(0.00886)\end{array}$ & $\begin{array}{c}0.0331 * * * \\
(0.00872)\end{array}$ & $\begin{array}{c}0.0360^{* * *} \\
(0.00824)\end{array}$ & $\begin{array}{c}0.0384 * * * \\
(0.00822)\end{array}$ & $\begin{array}{c}0.0393 * * * \\
(0.00819)\end{array}$ \\
\hline $\begin{array}{l}\text { Observations } \\
\text { R-squared }\end{array}$ & $\begin{array}{c}245 \\
0.163\end{array}$ & $\begin{array}{c}241 \\
0.158\end{array}$ & $\begin{array}{c}240 \\
0.170\end{array}$ & $\begin{array}{c}165 \\
0.149\end{array}$ & $\begin{array}{c}162 \\
0.148\end{array}$ & $\begin{array}{c}160 \\
0.152\end{array}$ & $\begin{array}{c}157 \\
0.163\end{array}$ & $\begin{array}{c}158 \\
0.148\end{array}$ & $\begin{array}{c}158 \\
0.139\end{array}$ & $\begin{array}{c}157 \\
0.131\end{array}$ \\
\hline
\end{tabular}

Standard errors in parentheses, *** $\mathrm{p}<0.01,{ }^{* *} \mathrm{p}<0.05,{ }^{*} \mathrm{p}<0.1$ 
Table A23: Evolution of growth post $10 \%$ episodes, controlling for education and saving rate, interaction with FDI dummy

\begin{tabular}{|c|c|c|c|c|c|c|c|c|c|c|}
\hline & (1) & (2) & (3) & (4) & (5) & (6) & (7) & (8) & (9) & (10) \\
\hline & GR1 & GR2 & GR3 & GR4 & GR5 & GR6 & GR7 & GR8 & GR9 & GR10 \\
\hline EPI*NFDI & $\begin{array}{l}-0.0220 \\
(0.0216)\end{array}$ & $\begin{array}{c}-0.0144 \\
(0.0190)\end{array}$ & $\begin{array}{l}-0.00356 \\
(0.0173)\end{array}$ & $\begin{array}{c}-0.0112 \\
(0.0160)\end{array}$ & $\begin{array}{l}-0.0141 \\
(0.0139)\end{array}$ & $\begin{array}{l}-0.0104 \\
(0.0131)\end{array}$ & $\begin{array}{l}-0.0117 \\
(0.0123)\end{array}$ & $\begin{array}{l}-0.00902 \\
(0.0115)\end{array}$ & $\begin{array}{l}-0.00859 \\
(0.0112)\end{array}$ & $\begin{array}{l}-0.00561 \\
(0.0109)\end{array}$ \\
\hline EPI*FDI & $\begin{array}{l}-0.00752 \\
(0.0123)\end{array}$ & $\begin{array}{l}-0.00516 \\
(0.0109)\end{array}$ & $\begin{array}{c}-0.00980 \\
(0.00989)\end{array}$ & $\begin{array}{c}-0.0152^{*} \\
(0.00912)\end{array}$ & $\begin{array}{c}-0.0167^{* *} \\
(0.00796)\end{array}$ & $\begin{array}{c}-0.0153^{* *} \\
(0.00747)\end{array}$ & $\begin{array}{c}-0.0139 * * \\
(0.00705)\end{array}$ & $\begin{array}{c}-0.0125^{*} \\
(0.00655)\end{array}$ & $\begin{array}{c}-0.0111^{*} \\
(0.00638)\end{array}$ & $\begin{array}{c}-0.0158^{* *} \\
(0.00726)\end{array}$ \\
\hline FDI & $\begin{array}{c}0.00742 \\
(0.00493)\end{array}$ & $\begin{array}{c}0.00934 * * \\
(0.00434)\end{array}$ & $\begin{array}{c}0.0120 * * * \\
(0.00395)\end{array}$ & $\begin{array}{c}0.0129 * * * \\
(0.00364)\end{array}$ & $\begin{array}{c}0.0113 * * * \\
(0.00318)\end{array}$ & $\begin{array}{c}0.0103 * * * \\
(0.00298)\end{array}$ & $\begin{array}{c}0.0102^{* * *} \\
(0.00281)\end{array}$ & $\begin{array}{c}0.00945 * * * \\
(0.00262)\end{array}$ & $\begin{array}{c}0.00807 * * * \\
(0.00255)\end{array}$ & $\begin{array}{c}0.00876 * * * \\
(0.00249)\end{array}$ \\
\hline LY & $\begin{array}{c}-0.00568 * * \\
(0.00227)\end{array}$ & $\begin{array}{c}-0.00626 * * * \\
(0.00200)\end{array}$ & $\begin{array}{c}-0.00592 * * * \\
(0.00182)\end{array}$ & $\begin{array}{c}-0.00489 * * * \\
(0.00168)\end{array}$ & $\begin{array}{c}-0.00593^{* * * *} \\
(0.00146)\end{array}$ & $\begin{array}{c}-0.00613^{* * *} \\
(0.00137)\end{array}$ & $\begin{array}{c}-0.00625^{* * *} \\
(0.00129)\end{array}$ & $\begin{array}{c}-0.00611^{* * * *} \\
(0.00120)\end{array}$ & $\begin{array}{c}-0.00556 * * * \\
(0.00117)\end{array}$ & $\begin{array}{c}-0.00527^{* * *} * \\
(0.00115)\end{array}$ \\
\hline iyr_sch & $\begin{array}{l}0.00219^{*} \\
(0.00123)\end{array}$ & $\begin{array}{c}0.00301 * * * \\
(0.00109)\end{array}$ & $\begin{array}{c}0.00332 * * * \\
(0.000990)\end{array}$ & $\begin{array}{c}0.00352 * * * \\
(0.000912)\end{array}$ & $\begin{array}{c}0.00394 * * * \\
(0.000796)\end{array}$ & $\begin{array}{c}0.00401^{* * *} \\
(0.000747)\end{array}$ & $\begin{array}{c}0.00416 * * * \\
(0.000705)\end{array}$ & $\begin{array}{c}0.00396 * * * \\
(0.000655)\end{array}$ & $\begin{array}{c}0.00338 * * * \\
(0.000638)\end{array}$ & $\begin{array}{c}0.00328 * * * \\
(0.000625)\end{array}$ \\
\hline Constant & $\begin{array}{c}0.0426 * * * \\
(0.0144)\end{array}$ & $\begin{array}{c}0.0399 * * * \\
(0.0127)\end{array}$ & $\begin{array}{c}0.0348 * * * \\
(0.0115)\end{array}$ & $\begin{array}{c}0.0277 * * * \\
(0.0106)\end{array}$ & $\begin{array}{c}0.0362 * * * \\
(0.00929)\end{array}$ & $\begin{array}{c}0.0398 * * * \\
(0.00871)\end{array}$ & $\begin{array}{c}0.0414^{* * *} \\
(0.00822)\end{array}$ & $\begin{array}{c}0.0414^{* * *} \\
(0.00765)\end{array}$ & $\begin{array}{c}0.0394 * * * \\
(0.00745)\end{array}$ & $\begin{array}{c}0.0377 * * * \\
(0.00731)\end{array}$ \\
\hline $\begin{array}{l}\text { Observations } \\
\text { R-squared }\end{array}$ & $\begin{array}{c}260 \\
0.042\end{array}$ & $\begin{array}{c}260 \\
0.072\end{array}$ & $\begin{array}{c}260 \\
0.104\end{array}$ & $\begin{array}{c}260 \\
0.141\end{array}$ & $\begin{array}{c}260 \\
0.181\end{array}$ & $\begin{array}{c}260 \\
0.191\end{array}$ & $\begin{array}{c}260 \\
0.216\end{array}$ & $\begin{array}{c}260 \\
0.219\end{array}$ & $\begin{array}{c}260 \\
0.177\end{array}$ & $\begin{array}{c}257 \\
0.189\end{array}$ \\
\hline & $\begin{array}{c}(11) \\
\text { GR11 }\end{array}$ & $\begin{array}{l}(12) \\
\text { GR12 }\end{array}$ & $\begin{array}{c}\text { (13) } \\
\text { GR13 }\end{array}$ & $\begin{array}{c}\text { (14) } \\
\text { GR14 }\end{array}$ & $\begin{array}{l}\text { (15) } \\
\text { GR15 }\end{array}$ & $\begin{array}{c}\text { (16) } \\
\text { GR16 }\end{array}$ & $\begin{array}{l}\text { (17) } \\
\text { GR17 }\end{array}$ & $\begin{array}{l}\text { (18) } \\
\text { GR18 }\end{array}$ & $\begin{array}{c}\text { (19) } \\
\text { GR19 }\end{array}$ & $\begin{array}{l}(20) \\
\text { GR20 }\end{array}$ \\
\hline EPI*NFDI & $\begin{array}{l}-0.00703 \\
(0.0108)\end{array}$ & $\begin{array}{l}-0.00462 \\
(0.0102)\end{array}$ & $\begin{array}{l}-0.00491 \\
(0.0101)\end{array}$ & $\begin{array}{c}-2.51 \mathrm{e}-06 \\
(0.0103)\end{array}$ & $\begin{array}{c}-0.000993 \\
(0.00971)\end{array}$ & $\begin{array}{c}-0.00325 \\
(0.00949)\end{array}$ & $\begin{array}{c}-0.00444 \\
(0.00916)\end{array}$ & $\begin{array}{c}-0.00352 \\
(0.00876)\end{array}$ & $\begin{array}{c}-0.00448 \\
(0.00871)\end{array}$ & $\begin{array}{c}-0.00254 \\
(0.00873)\end{array}$ \\
\hline EPI*FDI & $\begin{array}{c}-0.0144 * * \\
(0.00719)\end{array}$ & $\begin{array}{c}-0.0182^{* *} \\
(0.00734)\end{array}$ & $\begin{array}{c}-0.0181^{* *} \\
(0.00725)\end{array}$ & $\begin{array}{c}-0.0151^{* *} \\
(0.00756)\end{array}$ & $\begin{array}{c}-0.0161^{* *} \\
(0.00715)\end{array}$ & $\begin{array}{c}-0.0178 * * \\
(0.00697)\end{array}$ & $\begin{array}{c}-0.0192^{* * *} \\
(0.00675)\end{array}$ & $\begin{array}{l}-0.0164 * * \\
(0.00698)\end{array}$ & $\begin{array}{l}-0.0181^{* *} \\
(0.00767)\end{array}$ & $\begin{array}{l}-0.0161^{*} \\
(0.00876)\end{array}$ \\
\hline FDI & $\begin{array}{c}0.00763 * * * \\
(0.00246)\end{array}$ & $\begin{array}{c}0.00795 * * * \\
(0.00234)\end{array}$ & $\begin{array}{c}0.00791 * * * \\
(0.00232)\end{array}$ & $\begin{array}{c}0.00975 * * * \\
(0.00294)\end{array}$ & $\begin{array}{c}0.00925 * * * \\
(0.00278)\end{array}$ & $\begin{array}{c}0.00894 * * * \\
(0.00273)\end{array}$ & $\begin{array}{c}0.00934 * * * \\
(0.00268)\end{array}$ & $\begin{array}{c}0.00791 * * * \\
(0.00251)\end{array}$ & $\begin{array}{c}0.00662 * * * \\
(0.00250)\end{array}$ & $\begin{array}{c}0.00632 * * \\
(0.00250)\end{array}$ \\
\hline LY & $\begin{array}{c}-0.00579 * * * \\
(0.00114)\end{array}$ & $\begin{array}{c}-0.00559^{* * *} \\
(0.00108)\end{array}$ & $\begin{array}{c}-0.00610 * * * \\
(0.00107)\end{array}$ & $\begin{array}{c}-0.00292^{* *} \\
(0.00146)\end{array}$ & $\begin{array}{c}-0.00370 * * * \\
(0.00140)\end{array}$ & $\begin{array}{c}-0.00391^{* * *} \\
(0.00138)\end{array}$ & $\begin{array}{c}-0.00381^{* * *} \\
(0.00134)\end{array}$ & $\begin{array}{c}-0.00399 * * * \\
(0.00126)\end{array}$ & $\begin{array}{c}-0.00431^{* * *} \\
(0.00126)\end{array}$ & $\begin{array}{c}-0.00433^{* * *} \\
(0.00126)\end{array}$ \\
\hline iyr_sch & $\begin{array}{c}0.00348 * * * \\
(0.000619)\end{array}$ & $\begin{array}{c}0.00305 * * * \\
(0.000595)\end{array}$ & $\begin{array}{c}0.00316 * * * \\
(0.000588)\end{array}$ & $\begin{array}{c}0.00281 * * * \\
(0.000789)\end{array}$ & $\begin{array}{c}0.00294 * * * \\
(0.000751)\end{array}$ & $\begin{array}{c}0.00293 * * * \\
(0.000739)\end{array}$ & $\begin{array}{c}0.00281 * * * \\
(0.000718)\end{array}$ & $\begin{array}{c}0.00278 * * * \\
(0.000683)\end{array}$ & $\begin{array}{c}0.00281 * * * \\
(0.000681)\end{array}$ & $\begin{array}{c}0.00280^{* * *} \\
(0.000684)\end{array}$ \\
\hline Constant & $\begin{array}{c}0.0404 * * * \\
(0.00723)\end{array}$ & $\begin{array}{c}0.0411 * * * \\
(0.00685)\end{array}$ & $\begin{array}{c}0.0447 * * * \\
(0.00684)\end{array}$ & $\begin{array}{l}0.0204^{* *} \\
(0.00914)\end{array}$ & $\begin{array}{c}0.0273 * * * \\
(0.00870)\end{array}$ & $\begin{array}{c}0.0303^{* * *} \\
(0.00855)\end{array}$ & $\begin{array}{c}0.0309 * * * \\
(0.00838)\end{array}$ & $\begin{array}{c}0.0332 * * * \\
(0.00781)\end{array}$ & $\begin{array}{c}0.0351 * * * \\
(0.00777)\end{array}$ & $\begin{array}{c}0.0358 * * * \\
(0.00778)\end{array}$ \\
\hline $\begin{array}{l}\text { Observations } \\
\text { R-squared }\end{array}$ & $\begin{array}{c}257 \\
0.191\end{array}$ & $\begin{array}{c}253 \\
0.190\end{array}$ & $\begin{array}{c}251 \\
0.205\end{array}$ & $\begin{array}{c}169 \\
0.170\end{array}$ & $\begin{array}{c}167 \\
0.177\end{array}$ & $\begin{array}{c}165 \\
0.184\end{array}$ & $\begin{array}{c}162 \\
0.199\end{array}$ & $\begin{array}{c}164 \\
0.177\end{array}$ & $\begin{array}{c}164 \\
0.168\end{array}$ & $\begin{array}{c}163 \\
0.153\end{array}$ \\
\hline
\end{tabular}

Standard errors in parentheses, *** $\mathrm{p}<0.01, * * \mathrm{p}<0.05, * \mathrm{p}<0.1$ 